\title{
Quadruple Borylation of Terminal Alkynes
}

Daiki Yukimori, ${ }^{\dagger}$ Yuki Nagashima, ${ }^{,+}{ }^{\dagger}$ Chao Wang, ${ }^{\dagger, \neq}$

Atsuya Muranaka, ${ }^{\ddagger}$ and Masanobu Uchiyama ${ }^{*,+, \neq, \S}$

${ }^{\dagger}$ Graduate School of Pharmaceutical Sciences, The University of Tokyo, 7-3-1 Hongo, Bunkyo-ku, Tokyo 113-0033, Japan

${ }^{\ddagger}$ Cluster for Pioneering Research (CPR), Advanced Elements Chemistry Research Team, RIKEN, 2-1 Hirosawa, Wako, Saitama 351-0198, Japan

${ }^{\S}$ Research Initiative for Supra-Materials (RISM), Shinshu University, Ueda, Nagano 386-8567, Japan

\section{Table of Contents}

1. General

S-2

2. Experimental Section

3. Computational Details

S-15

4. Single Crystal X-ray Structure

5. References

S-25

6. Copies of NMR Spectra 


\section{General}

\section{Instrumentation.}

NMR spectra were obtained on a BRUKER AVANCE III HD 500 spectrometer. Chemical shifts are expressed in $\delta(\mathrm{ppm})$ values and coupling constants are expressed in hertz $(\mathrm{Hz}) .{ }^{1} \mathrm{H},{ }^{13} \mathrm{C}$ NMR spectra were referenced to tetramethylsilane as an internal standard. The following abbreviations are used: $\mathrm{s}=$ singlet, $\mathrm{d}=$ doublet, $\mathrm{t}=$ triplet, $\mathrm{m}=$ multiplet, and $\mathrm{bs}=$ broad singlet. Melting points were determined with a Yanaco micro melting points apparatus or a SRS MPA 100 OptiMelt automated melting point system and are uncorrected. ESI mass spectra were measured on a Bruker micrOTOF-II spectrometer. IR spectra were obtained on a JASCO FR/IR-4700 spectrometer.

\section{Materials.}

Unless otherwise noted, materials were purchased from Aldrich Inc., Wako Pure Chemical Industries, Ltd., Tokyo Kasei Co., and other commercial suppliers and were used after appropriate purification. All chemicals were of reagent grade and used as received. Air- and moisture-sensitive manipulations were performed with standard Schlenk techniques under argon atmosphere. Photoreactions were performed in a Schlenck tube using a $430 \mathrm{~mW} / \mathrm{cm}^{2}$ medium-pressure Hg lamp (YMC-P-0066) equipped with a cooling fan. Normal-phase column chromatography was performed with YAMAZEN YFLC AI-580.

NOTE: Normal-phase column chromatography was performed within 30 minutes, since many of 1,1,2,2-tetrakis(boronate) derivatives (3) are not so stable on silica gel. 


\section{Experimental Section}

\subsection{Multi-borylation of Terminal Alkynes (Table 2)}

Typical Procedure A:

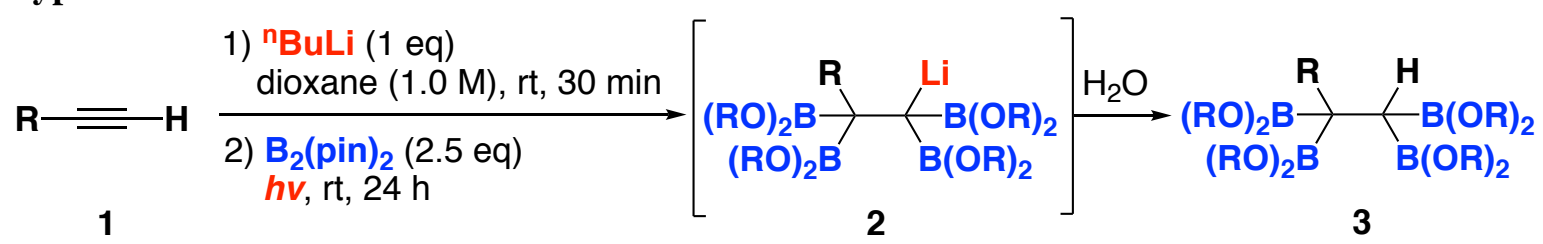

Terminal alkyne $1(0.25 \mathrm{mmol})$ was charged in a dried Schlenk tube, and dissolved in dry dioxane $(0.25 \mathrm{~mL}, 1.0$ M). To the mixture was added ${ }^{\mathrm{n}} \mathrm{BuLi}\left(0.09 \mathrm{~mL}, 0.25 \mathrm{mmol} ; 2.60 \mathrm{M}\right.$ in hexane solution) at $0^{\circ} \mathrm{C}$, and the solution was stirred for $15 \mathrm{~min}$. To the mixture was successively added diboron $(0.625 \mathrm{mmol})$ at room temperature. After the reaction tube was sealed and irradiated for 24 hours with a $430 \mathrm{~mW} / \mathrm{cm}^{-1}$ medium-pressure $\mathrm{Hg}$ lamp equipped with a cooling fan. The reaction was quenched by $\mathrm{H}_{2} \mathrm{O}(1 \mathrm{~mL})$, and then extracted with AcOEt $(15 \mathrm{~mL}$ $\times 3$ ). The AcOEt layers were combined and dried over $\mathrm{MgSO}_{4}$, and the solvent was removed under reduced pressure. The residue was purified by silica gel column chromatography using hexane/AcOEt as eluent to give the 1,1,2,2-tetrakis(boronate) compound (3).

2,2',2",2'"'-(1-(4-methoxyphenyl)ethane-1,1,2,2-tetrayl)tetrakis(4,4,5,5-tetramethyl-1,3,2-dioxaborolane)

(3a)

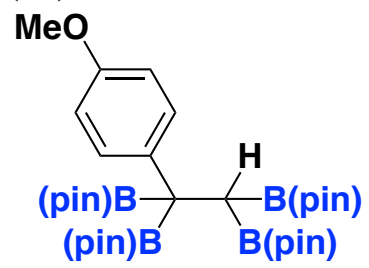

Using the Typical Procedure A, the titled compound was obtained as a white powder in $90 \%$ yield (144 $\mathrm{mg}$ ). ${ }^{1} \mathrm{H}$ NMR $\left(\mathrm{CDCl}_{3}, 500 \mathrm{MHz}\right): 7.43(\mathrm{~d}, 2 \mathrm{H}, J=4.5 \mathrm{~Hz}), 6.71(\mathrm{~d}, 2 \mathrm{H}, J=4.5 \mathrm{~Hz}), 3.74(\mathrm{~s}, 3 \mathrm{H}), 1.54(\mathrm{~s}, 1 \mathrm{H}), 1.22(\mathrm{~s}$,

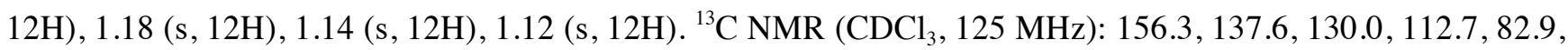
$82.5,55.1,25.0,24.9,24.5,24.4$. The carbon directly attached to the boron atom was not detected, likely due to quadropolar relaxation. ${ }^{11} \mathrm{~B} \mathrm{NMR}\left(\mathrm{CDCl}_{3}, 160 \mathrm{MHz}\right)$ : 34.6. $\mathrm{mp}: 160.5-162.8^{\circ} \mathrm{C}$ (recrystallized from hexane).

$\mathrm{C}_{33} \mathrm{H}_{56} \mathrm{~B}_{4} \mathrm{O}_{9}$. HRMS (pos. ESI) $\mathrm{m} / z$ : calcd for $[\mathrm{M}+\mathrm{Na}]^{+}$663.4194, found 663.4144 . ATR-FTIR (neat) $v: 2978,2365,1509,1298,1242,1139,1038,970,849,776,730,556,492,481,466,447$, $435,426 \mathrm{~cm}^{-1}$.

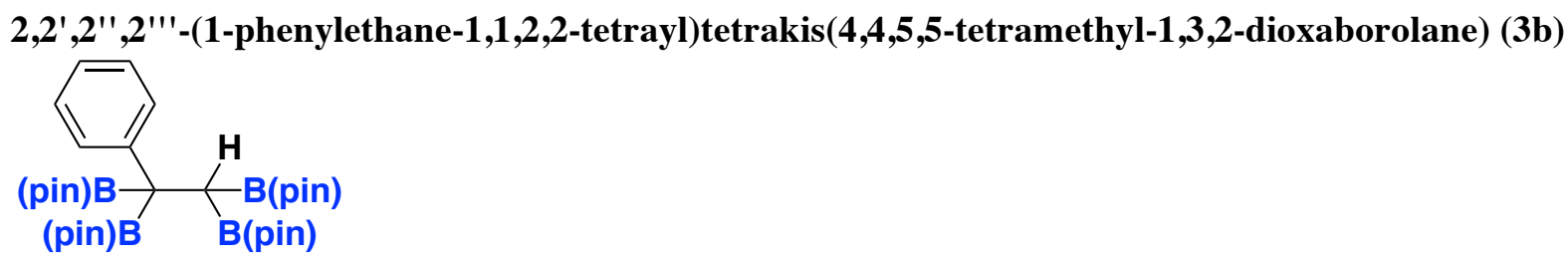

Using the Typical Procedure A, the titled compound was obtained as a white powder in $82 \%$ yield (142 $\mathrm{mg}$, including $\left.12 \mathrm{wt} \% \mathrm{~B}_{2}(\mathrm{pin})_{2}\right)$.

${ }^{1} \mathrm{H} \mathrm{NMR}\left(\mathrm{CDCl}_{3}, 500 \mathrm{MHz}\right): 7.51(\mathrm{dd}, 1 \mathrm{H}, J=7.5,1.5 \mathrm{~Hz}), 7.14(\mathrm{dd}, 2 \mathrm{H}, J=7.5,7.5 \mathrm{~Hz}), 7.00-6.97(\mathrm{~m}, 1 \mathrm{H})$, 
$1.38(\mathrm{~s}, 1 \mathrm{H}), 1.22(\mathrm{~s}, 12 \mathrm{H}), 1.18(\mathrm{~s}, 12 \mathrm{H}), 1.15(\mathrm{~s}, 12 \mathrm{H}), 1.12(\mathrm{~s}, 12 \mathrm{H}) .{ }^{13} \mathrm{C} \mathrm{NMR}\left(\mathrm{CDCl}_{3}, 125 \mathrm{MHz}\right): 145.5$, $129.0,127.1,123.8,83.2,82.9,82.5,24.9,24.9,24.6,24.5,24.4$. The carbon directly attached to the boron atom was not detected, likely due to quadropolar relaxation. ${ }^{11} \mathrm{~B} \mathrm{NMR}\left(\mathrm{CDCl}_{3}, 160 \mathrm{MHz}\right): 33.9,30.2$.

$\mathrm{C}_{32} \mathrm{H}_{54} \mathrm{~B}_{4} \mathrm{O}_{8}$. HRMS (pos. ESI) $m / z$ : calcd for $[\mathrm{M}+\mathrm{Na}]^{+}$633.4089, found 633.4077 .

ATR-FTIR (neat) $v: 2977,1457,1298,1213,1135,970,847,698,668,564,534,498,485,473,439,422 \mathrm{~cm}^{-1}$.

\section{2,2',2",2'"'-(1-(3-methoxyphenyl)ethane-1,1,2,2-tetrayl)tetrakis (4,4,5,5-tetramethyl-1,3,2-dioxaborolane)}

(3c)

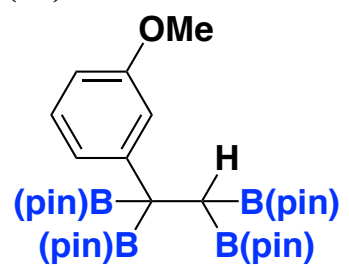

Using the Typical Procedure A, the titled compound was obtained as a white powder in $87 \%$ yield (139 $\mathrm{mg})$. ${ }^{1} \mathrm{H} \mathrm{NMR}\left(\mathrm{CDCl}_{3}, 500 \mathrm{MHz}\right): 7.17(\mathrm{dd}, 1 \mathrm{H}, J=2.1,2.1 \mathrm{~Hz}), 7.12(\mathrm{~d}, 1 \mathrm{H}, J=7.5 \mathrm{~Hz}), 7.07(\mathrm{dd}, 1 \mathrm{H}, J=7.5,7.5$ $\mathrm{Hz}), 6.56(\mathrm{dd}, 1 \mathrm{H}, J=7.0,2.1 \mathrm{~Hz}), 3.76(\mathrm{~s}, 3 \mathrm{H}), 1.58(\mathrm{~s}, 1 \mathrm{H}), 1.25(\mathrm{~s}, 12 \mathrm{H}), 1.22(\mathrm{~s}, 12 \mathrm{H}), 1.18(\mathrm{~s}, 12 \mathrm{H}), 1.15(\mathrm{~s}$, 12H). ${ }^{13} \mathrm{C} \mathrm{NMR}\left(\mathrm{CDCl}_{3}, 125 \mathrm{MHz}\right): 158.6,147.1,127.9,121.6,114.3,110.4,82.9,82.5,54.9,25.0,24.9,24.5$, 24.4. The carbon directly attached to the boron atom was not detected, likely due to quadropolar relaxation. ${ }^{11} \mathrm{~B}$ NMR $\left(\mathrm{CDCl}_{3}, 160 \mathrm{MHz}\right): 33.0,30.2 . \mathrm{mp}: 99.0-100.4^{\circ} \mathrm{C}$ (recrystallized from hexane).

$\mathrm{C}_{33} \mathrm{H}_{56} \mathrm{~B}_{4} \mathrm{O}_{9}$. HRMS (pos. ESI) $\mathrm{m} / z$ : calcd for $[\mathrm{M}+\mathrm{Na}]^{+} 663.4194$, found 663.4145 .

ATR-FTIR (neat) $v: 2974,1296,1137,1047,975,849,780,701,662,577,480,471,444,423 \mathrm{~cm}^{-1}$.

2,2',2",2'"'-(1-(2-methoxyphenyl)ethane-1,1,2,2-tetrayl)tetrakis(4,4,5,5-tetramethyl-1,3,2-dioxaborolane) (3d)

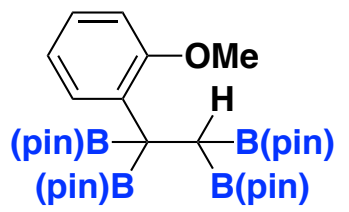

Using the Typical Procedure A, the titled compound was obtained as a colorless amorphous in $87 \%$ yield (132 $\mathrm{mg})$.

${ }^{1} \mathrm{H}$ NMR $\left(\mathrm{CDCl}_{3}, 500 \mathrm{MHz}\right): 7.36(\mathrm{dd}, 1 \mathrm{H}, J=7.7,1.6 \mathrm{~Hz}), 7.00(\mathrm{ddd}, 1 \mathrm{H}, J=7.8,7.8,1.6 \mathrm{~Hz}), 6.74$ (ddd, $1 \mathrm{H}, J$ $=7.8,7.7,1.6 \mathrm{~Hz}), 6.66(\mathrm{dd}, 1 \mathrm{H}, J=7.8,1.6 \mathrm{~Hz}), 3.72(\mathrm{~s}, 3 \mathrm{H}), 1.50(\mathrm{~s}, 1 \mathrm{H}), 1.25(\mathrm{~s}, 12 \mathrm{H}), 1.23(\mathrm{~s}, 12 \mathrm{H}), 1.13(\mathrm{~s}$, $12 \mathrm{H}), 1.11$ (s, 12H). ${ }^{13} \mathrm{C} \mathrm{NMR}\left(\mathrm{CDCl}_{3}, 125 \mathrm{MHz}\right): 157.6,135.3,131.5,125.4,119.7,114.3,109.4,82.8,82.1$, $54.7,25.2,25.0,24.8,24.7$. The carbon directly attached to the boron atom was not detected, likely due to quadropolar relaxation. ${ }^{11} \mathrm{~B} \mathrm{NMR}\left(\mathrm{CDCl}_{3}, 160 \mathrm{MHz}\right): 34.7,31.2$.

$\mathrm{C}_{33} \mathrm{H}_{56} \mathrm{~B}_{4} \mathrm{O}_{9}$. HRMS (pos. ESI) $m / z$ : calcd for $[\mathrm{M}+\mathrm{Na}]^{+}$663.4194, found 663.4208 .

ATR-FTIR (neat) $v: 2971,1297,1242,1138,1109,1024,971,851,748,684,664,590,555,485,456,439$ $\mathrm{cm}^{-1}$.

N,N-diphenyl-4-(1,1,2,2-tetrakis(4,4,5,5-tetramethyl-1,3,2-dioxaborolan-2-yl)ethyl)aniline (3e) 


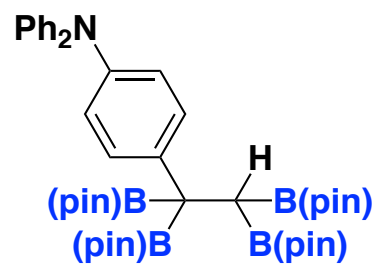

Using the Typical Procedure A, the titled compound was obtained as a white powder in $68 \%$ yield (256 $\mathrm{mg}$, $0.50 \mathrm{mmol} \mathrm{scal})$.

${ }^{1} \mathrm{H}$ NMR $\left(\mathrm{CDCl}_{3}, 500 \mathrm{MHz}\right): 7.48$ (d, 2H, $\left.J=8.5 \mathrm{~Hz}\right), 7.17-7.14(\mathrm{~m}, 4 \mathrm{H}), 7.04-7.02(\mathrm{~m}, 4 \mathrm{H}), 6.92-6.90(\mathrm{~m}, 4 \mathrm{H})$, $1.28(\mathrm{~s}, 1 \mathrm{H}), 1.24(\mathrm{~s}, 12 \mathrm{H}), 1.21(\mathrm{~s}, 12 \mathrm{H}), 1.14(\mathrm{~s}, 12 \mathrm{H}), 1.13(\mathrm{~s}, 12 \mathrm{H}) .{ }^{13} \mathrm{C}$ NMR $\left(\mathrm{CDCl}_{3}, 125 \mathrm{MHz}\right): 143.6$, $133.6,131.2,129.5,127.7,127.1,125.9,125.4,124.7,124.0,83.0,82.7,24.9,24.8,24.6,24.5$. The carbon directly attached to the boron atom was not detected, likely due to quadropolar relaxation. ${ }^{11} \mathrm{~B} \mathrm{NMR}\left(\mathrm{CDCl}_{3}, 160\right.$ $\mathrm{MHz}$ ): $34.5,34.4$. $\mathrm{mp}: 163.0-165.3^{\circ} \mathrm{C}$ (recrystallized from hexane).

$\mathrm{C}_{44} \mathrm{H}_{63} \mathrm{~B}_{4} \mathrm{NO}_{8}$. HRMS (pos. ESI) $\mathrm{m} / z$ : calcd for $[\mathrm{M}+\mathrm{H}]^{+} 778.5004$, found 778.4998 .

ATR-FTIR (neat) $v: 2978,1489,1295,1138,974,848,755,727,696,669,572,526,470,461,433 \mathrm{~cm}^{-1}$.

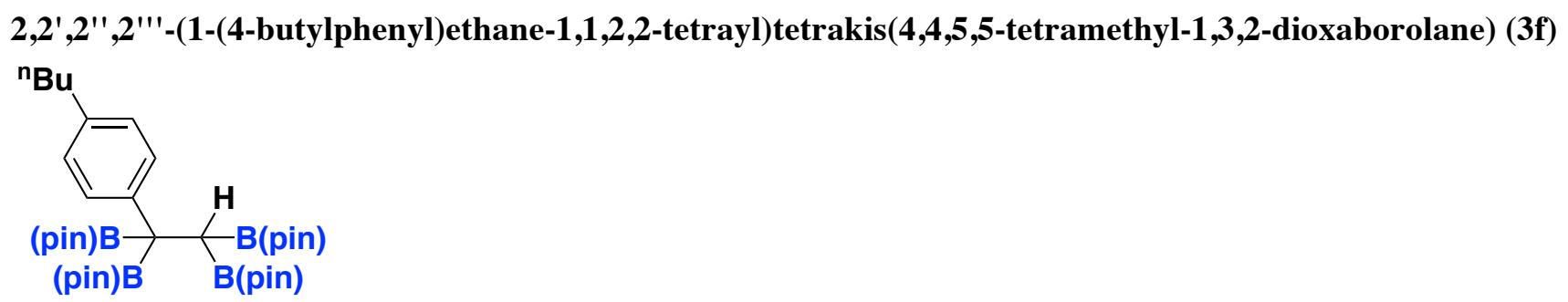

Using the Typical Procedure A, the titled compound was obtained as a white powder in 54\% yield (179 $\mathrm{mg}$, $0.50 \mathrm{mmol} \mathrm{scal})$.

${ }^{1} \mathrm{H}$ NMR $\left(\mathrm{CDCl}_{3}, 500 \mathrm{MHz}\right): 7.40(\mathrm{~d}, 2 \mathrm{H}, J=7.5 \mathrm{~Hz}), 6.93(\mathrm{~d}, 2 \mathrm{H}, J=7.5 \mathrm{~Hz}), 2.49(\mathrm{t}, 2 \mathrm{H}, J=7.5 \mathrm{~Hz})$, 1.53-1.48 (m, 2H), 1.30-1.26 (m, 2H), $1.28(\mathrm{~s}, 1 \mathrm{H}), 1.22(\mathrm{~s}, 12 \mathrm{H}), 1.18(\mathrm{~s}, 12 \mathrm{H}), 1.13(\mathrm{~s}, 12 \mathrm{H}), 1.11(\mathrm{~s}, 12 \mathrm{H}) .{ }^{13} \mathrm{C}$ NMR $\left(\mathrm{CDCl}_{3}, 125 \mathrm{MHz}\right): 142.4,137.9,128.9,127.3,82.8,82.5,35.1,33.7,25.0,24.9,24.5,24.4,22.3,14.0$. The carbon directly attached to the boron atom was not detected, likely due to quadropolar relaxation. ${ }^{11} \mathrm{~B}$ NMR $\left(\mathrm{CDCl}_{3}, 160 \mathrm{MHz}\right): 33.9 . \mathrm{mp}: 126.8-128.0^{\circ} \mathrm{C}$ (recrystallized from hexane).

$\mathrm{C}_{36} \mathrm{H}_{62} \mathrm{~B}_{4} \mathrm{O}_{8}$. HRMS (pos. ESI) $m / z$ : calcd for $[\mathrm{M}+\mathrm{Na}]^{+} 689.4715$, found 689.4724 .

ATR-FTIR (neat) $v: 2976,2364,2360,1295,1265,1213,1138,970,849,750,666,580,494,470,458,447$, $429 \mathrm{~cm}^{-1}$.

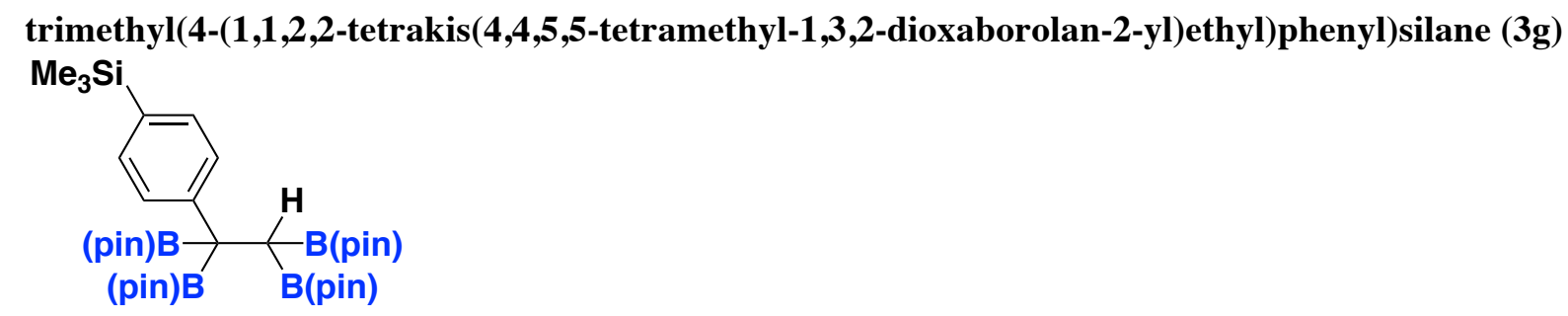

Using the Typical Procedure A, the titled compound was obtained as a white powder in $88 \%$ yield (310 $\mathrm{mg}$, $0.50 \mathrm{mmol} \mathrm{scal})$.

${ }^{1} \mathrm{H}$ NMR $\left(\mathrm{CDCl}_{3}, 500 \mathrm{MHz}\right): 7.47$ (d, 2H, $\left.J=8.0 \mathrm{~Hz}\right), 7.27$ (d, 2H, J = 8.0 Hz), $1.29(\mathrm{~s}, 1 \mathrm{H}), 1.24(\mathrm{~s}, 12 \mathrm{H}), 1.19$ 
$(\mathrm{s}, 12 \mathrm{H}), 1.14(\mathrm{~s}, 12 \mathrm{H}), 1.12(\mathrm{~s}, 12 \mathrm{H}), 0.18(\mathrm{~s}, 9 \mathrm{H}) .{ }^{13} \mathrm{C} \mathrm{NMR}\left(\mathrm{CDCl}_{3}, 125 \mathrm{MHz}\right): 146.2,134.3,132.4,128.4$, $82.9,82.5,25.0,24.9,24.5,24.4,-0.98$. The carbon directly attached to the boron atom was not detected, likely due to quadropolar relaxation. ${ }^{11} \mathrm{~B}$ NMR $\left(\mathrm{CDCl}_{3}, 160 \mathrm{MHz}\right)$ : 34.1, 33.9. mp: $158.3-160.1{ }^{\circ} \mathrm{C}$ (recrystallized from hexane).

$\mathrm{C}_{35} \mathrm{H}_{62} \mathrm{~B}_{4} \mathrm{O}_{8} \mathrm{Si}$. HRMS (pos. ESI) $\mathrm{m} / \mathrm{z}$ : calcd for $[\mathrm{M}+\mathrm{Na}]^{+} 705.4484$, found 705.4498 .

ATR-FTIR (neat) $v: 2978,1297,1251,1139,1109,971,836,728,670,638,573,512,464,439 \mathrm{~cm}^{-1}$.

2,2',2",2"'-(1-(3,5-dimethoxyphenyl)ethane-1,1,2,2-tetrayl)tetrakis(4,4,5,5-tetramethyl-1,3,2-dioxaborolane ) (3h)

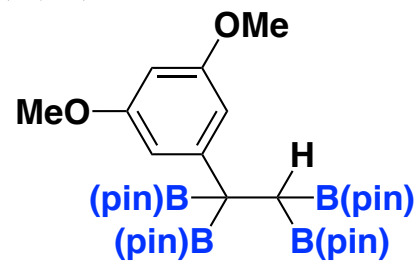

Using the Typical Procedure A, the titled compound was obtained as a white powder in $88 \%$ yield (274 $\mathrm{mg}$, $0.50 \mathrm{mmol} \mathrm{scal})$.

${ }^{1} \mathrm{H} \mathrm{NMR}\left(\mathrm{CDCl}_{3}, 500 \mathrm{MHz}\right): 6.76(\mathrm{~d}, 2 \mathrm{H}, J=2.0 \mathrm{~Hz}), 6.15(\mathrm{~m}, 1 \mathrm{H}), 3.74(\mathrm{~m}, 3 \mathrm{H}), 1.30(\mathrm{~s}, 1 \mathrm{H}), 1.23(\mathrm{~s}, 12 \mathrm{H})$, $1.20(\mathrm{~s}, 12 \mathrm{H}), 1.16(\mathrm{~s}, 12 \mathrm{H}), 1.14(\mathrm{~s}, 12 \mathrm{H}) .{ }^{13} \mathrm{C} \mathrm{NMR}\left(\mathrm{CDCl}_{3}, 125 \mathrm{MHz}\right): 159.6,147.9,107.3,97.5,82.9,82.6$, $55.0,25.0,24.9,24.6,24.5$. The carbon directly attached to the boron atom was not detected, likely due to quadropolar relaxation. ${ }^{11} \mathrm{~B}$ NMR $\left(\mathrm{CDCl}_{3}, 160 \mathrm{MHz}\right): 34.2,30.9 . \mathrm{mp}: 126.8-130.8^{\circ} \mathrm{C}$ (recrystallized from hexane).

$\mathrm{C}_{34} \mathrm{H}_{58} \mathrm{~B}_{4} \mathrm{O}_{10}$. HRMS (pos. ESI) $\mathrm{m} / \mathrm{z}$ : calcd for $[\mathrm{M}+\mathrm{Na}]^{+}$693.4300, found 693.4305 .

ATR-FTIR (neat) $v: 2971,1595,1295,1265,1204,1136,1089,1062,971,852,719,669,574,484,464,444$, $430 \mathrm{~cm}^{-1}$.

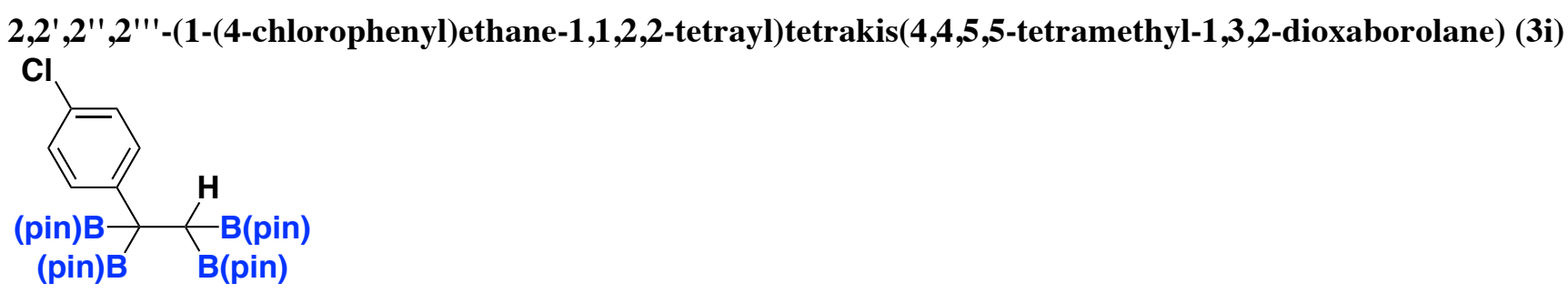

Using the Typical Procedure A, the titled compound was obtained as a colorless amorphous in 54\% yield (173 $\mathrm{mg}, 0.50 \mathrm{mmol} \mathrm{scal})$.

${ }^{1} \mathrm{H}$ NMR $\left(\mathrm{CDCl}_{3}, 500 \mathrm{MHz}\right): 7.45(\mathrm{~d}, 2 \mathrm{H}, J=8.5 \mathrm{~Hz}), 7.11(\mathrm{~d}, 2 \mathrm{H}, J=8.5 \mathrm{~Hz}), 1.27(\mathrm{~s}, 1 \mathrm{H}), 1.21(\mathrm{~s}, 12 \mathrm{H}), 1.18$ $(\mathrm{s}, 12 \mathrm{H}), 1.15(\mathrm{~s}, 12 \mathrm{H}), 1.12$ (s, 12H). ${ }^{13} \mathrm{C} \mathrm{NMR}\left(\mathrm{CDCl}_{3}, 125 \mathrm{MHz}\right): 130.4,130.2,127.1,119.9,83.1,82.7,25.0$, $24.9,24.5,24.4$. The carbon directly attached to the boron atom was not detected, likely due to quadropolar relaxation. ${ }^{11} \mathrm{~B}$ NMR $\left(\mathrm{CDCl}_{3}, 160 \mathrm{MHz}\right): 34.6$.

$\mathrm{C}_{32} \mathrm{H}_{53} \mathrm{~B}_{4} \mathrm{ClO}_{8}$. HRMS (pos. ESI) $\mathrm{m} / \mathrm{z}$ : calcd for $[\mathrm{M}+\mathrm{Na}]^{+} 667.3699$, found 667.3702 .

ATR-FTIR (neat) $v: 3902,2974,1540,1295,1138,848,734,708,670,660,607,572,524,480,467,426 \mathrm{~cm}^{-1}$.

2,2',2",2"'-(1-(3-chlorophenyl)ethane-1,1,2,2-tetrayl)tetrakis(4,4,5,5-tetramethyl-1,3,2-dioxaborolane) (3j) 


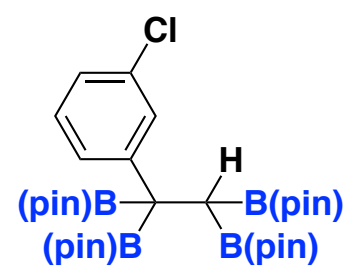

Using the Typical Procedure A, the titled compound was obtained as a white powder in $61 \%$ yield (196 $\mathrm{mg}$, $0.50 \mathrm{mmol} \mathrm{scal})$.

${ }^{1} \mathrm{H}$ NMR $\left(\mathrm{CDCl}_{3}, 500 \mathrm{MHz}\right): 7.50(\mathrm{dd}, 1 \mathrm{H}, J=2.4,2.4 \mathrm{~Hz}), 7.42(\mathrm{dd}, 1 \mathrm{H}, J=9.9,1.6 \mathrm{~Hz}), 7.07(\mathrm{dd}, 1 \mathrm{H}, J=9.9$, $9.9 \mathrm{~Hz}), 7.00-6.96(\mathrm{~m}, 1 \mathrm{H}), 1.26(\mathrm{~s}, 1 \mathrm{H}), 1.23(\mathrm{~s}, 12 \mathrm{H}), 1.20(\mathrm{~s}, 12 \mathrm{H}), 1.16(\mathrm{~s}, 12 \mathrm{H}), 1.14(\mathrm{~s}, 12 \mathrm{H}) .{ }^{13} \mathrm{C} \mathrm{NMR}$ $\left(\mathrm{CDCl}_{3}, 125 \mathrm{MHz}\right)$ : 132.9, 129.3, 128.2, 127.8, 127.1, 124.0, 83.1, 82.7, 24.9, 24.8, 24.5, 24.4. The carbon directly attached to the boron atom was not detected, likely due to quadropolar relaxation. ${ }^{11} \mathrm{~B} \mathrm{NMR}\left(\mathrm{CDCl}_{3}, 160\right.$ $\mathrm{MHz}$ ): 34.0. mp: $137.8-138.6^{\circ} \mathrm{C}$ (recrystallized from hexane).

$\mathrm{C}_{32} \mathrm{H}_{53} \mathrm{~B}_{4} \mathrm{ClO}_{8}$. HRMS (pos. ESI) $\mathrm{m} / \mathrm{z}$ : calcd for $[\mathrm{M}+\mathrm{Na}]^{+} 667.3699$, found 667.3702 .

ATR-FTIR (neat) $v: 2976,2364,2356,1484,1294,1215,1139,970,848,668,566,482,468,431,405 \mathrm{~cm}^{-1}$.

2,2',2',2'"'-(1-(4'-propyl-[1,1'-biphenyl]-4-yl)ethane-1,1,2,2-tetrayl)tetrakis(4,4,5,5-tetramethyl-1,3,2-dioxa borolane) (3k)

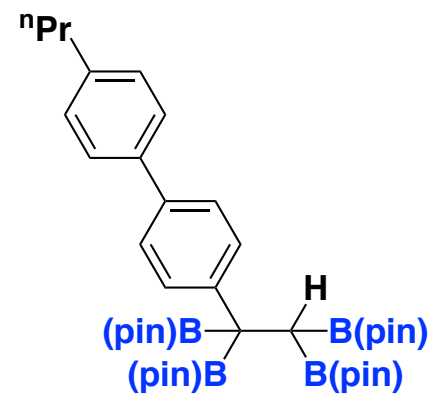

Using the Typical Procedure A, the titled compound was obtained as a white powder in 74\% yield (134 mg). ${ }^{1} \mathrm{H}$ NMR $\left(\mathrm{CDCl}_{3}, 500 \mathrm{MHz}\right): 7.56(\mathrm{~d}, 2 \mathrm{H}, J=9.0 \mathrm{~Hz}), 7.50(\mathrm{~d}, 2 \mathrm{H}, J=8.5 \mathrm{~Hz}), 7.39$ (d, 2H, J = 9.0 Hz), 7.19 (d, $2 \mathrm{H}, J=8.5 \mathrm{~Hz}), 2.60(\mathrm{t}, 2 \mathrm{H}, J=7.0 \mathrm{~Hz}), 1.66(\mathrm{tq}, 2 \mathrm{H}, J=7.5,7.0 \mathrm{~Hz}), 1.26(\mathrm{~s}, 1 \mathrm{H}), 1.23(\mathrm{~s}, 12 \mathrm{H}), 1.20(\mathrm{~s}, 12 \mathrm{H})$, $1.15(\mathrm{~s}, 12 \mathrm{H}), 1.13(\mathrm{~s}, 12 \mathrm{H}), 0.96(\mathrm{t}, 3 \mathrm{H}, J=7.5 \mathrm{~Hz}) .{ }^{13} \mathrm{C} \mathrm{NMR}\left(\mathrm{CDCl}_{3}, 125 \mathrm{MHz}\right): 144.5,140.8,139.2,136.2$, $129.3,128.7,126.6,125.8,83.0,82.6,37.7,25.0,24.9,24.6,24.5,24.4,13.9$. The carbon directly attached to the boron atom was not detected, likely due to quadropolar relaxation. ${ }^{11} \mathrm{~B} \mathrm{NMR}\left(\mathrm{CDCl}_{3}, 160 \mathrm{MHz}\right)$ : 33.6, 33.2. mp: 149.1-150. $3^{\circ} \mathrm{C}$ (recrystallized from hexane).

$\mathrm{C}_{41} \mathrm{H}_{64} \mathrm{~B}_{4} \mathrm{O}_{8}$. HRMS (pos. ESI) $m / z$ : calcd for $[\mathrm{M}+\mathrm{Na}]^{+} 751.4871$, found 751.4898 .

ATR-FTIR (neat) $v: 2972,1300,1213,1137,974,847,806,740,684,669,656,571,541,494,460,444 \mathrm{~cm}^{-1}$.

2,2',2",2'"'-(1-(4-(phenylethynyl)phenyl)ethane-1,1,2,2-tetrayl)tetrakis(4,4,5,5-tetramethyl-1,3,2-dioxaborol ane) (31) 


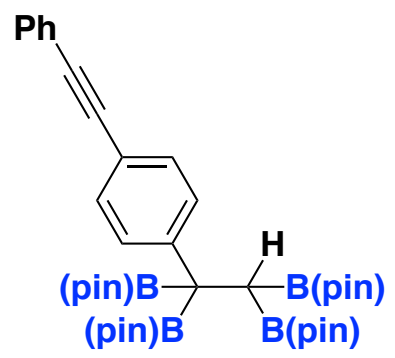

Using the Typical Procedure A, the titled compound was obtained as a white powder in $68 \%$ yield (241 $\mathrm{mg}$, $0.50 \mathrm{mmol}$ scal).

${ }^{1} \mathrm{H}$ NMR $\left(\mathrm{CDCl}_{3}, 500 \mathrm{MHz}\right)$ : 7.52-7.49 (m, 4H), 7.35-7.26 (m, 5H), $1.32(\mathrm{~s}, 1 \mathrm{H}), 1.21(\mathrm{~s}, 12 \mathrm{H}), 1.18(\mathrm{~s}, 12 \mathrm{H})$, $1.16(\mathrm{~s}, 12 \mathrm{H}), 1.13$ (s, 12H). ${ }^{13} \mathrm{C} \mathrm{NMR}\left(\mathrm{CDCl}_{3}, 125 \mathrm{MHz}\right): 131.5,130.6,130.3,128.9,128.8,128.2,127.9,127.7$, $83.5,83.0,82.7,77.6,25.0,24.8,24.5,24.4$. The carbon directly attached to the boron atom was not detected, likely due to quadropolar relaxation. ${ }^{11} \mathrm{~B} \mathrm{NMR}\left(\mathrm{CDCl}_{3}, 160 \mathrm{MHz}\right)$ : 30.7, 30.3 . mp: 202.5-206.3 ${ }^{\circ} \mathrm{C}$ (recrystallized from hexane).

$\mathrm{C}_{40} \mathrm{H}_{58} \mathrm{~B}_{4} \mathrm{O}_{8}$. HRMS (pos. ESI) $m / z$ : calcd for $[\mathrm{M}+\mathrm{Na}]^{+} 733.4402$, found 733.4413 .

ATR-FTIR (neat) $v: 2975,1488,1295,1137,972,848,757,698,670,574,513,479,467,440 \mathrm{~cm}^{-1}$.

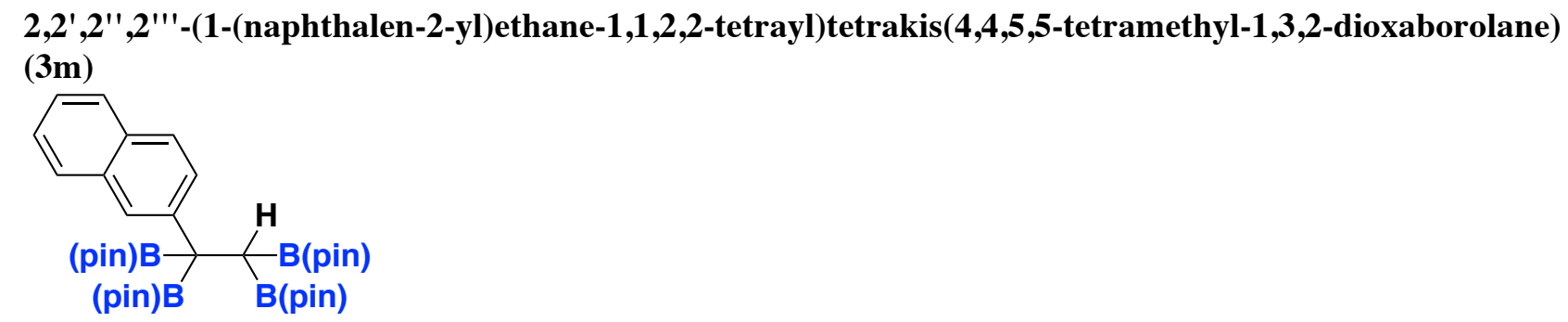

Using the Typical Procedure A, the titled compound was obtained as a white powder in $92 \%$ yield (303 $\mathrm{mg}$, $0.50 \mathrm{mmol} \mathrm{scal})$.

${ }^{1} \mathrm{H} \mathrm{NMR}\left(\mathrm{CDCl}_{3}, 500 \mathrm{MHz}\right)$ : 7.79-7.76 (m, 2H), 7.71-7.67 (m, 2H), $7.63(\mathrm{~d}, 1 \mathrm{H}, J=8.5 \mathrm{~Hz}), 7.35-7.28(\mathrm{~m}, 2 \mathrm{H})$, $1.42(\mathrm{~s}, 1 \mathrm{H}), 1.22(\mathrm{~s}, 12 \mathrm{H}), 1.19(\mathrm{~s}, 12 \mathrm{H}), 1.17(\mathrm{~s}, 12 \mathrm{H}), 1.12(\mathrm{~s}, 12 \mathrm{H}),{ }^{13} \mathrm{C}$ NMR $\left(\mathrm{CDCl}_{3}, 125 \mathrm{MHz}\right): 143.6$, 133.6, 131.2, 129.5, 127.7, 127.1, 125.9, 125.4, 124.7, 124.0, 83.0, 82.7, 24.9, 24.9, 24.6, 24.5. The carbon directly attached to the boron atom was not detected, likely due to quadropolar relaxation. ${ }^{11} \mathrm{~B} \mathrm{NMR}\left(\mathrm{CDCl}_{3}, 160\right.$ $\mathrm{MHz}$ ): $34.7,34.2 \mathrm{mp}: 162.1-168.3^{\circ} \mathrm{C}$ (recrystallized from hexane).

$\mathrm{C}_{36} \mathrm{H}_{56} \mathrm{~B}_{4} \mathrm{O}_{8}$. HRMS (pos. ESI) $\mathrm{m} / \mathrm{z}$ : calcd for $[\mathrm{M}+\mathrm{Na}]^{+} 683.4245$, found 683.4301 . ATR-FTIR (neat) $v: 2973,1304,1265,1213,1137,972,850,738,664,573,477,452,437 \mathrm{~cm}^{-1}$.

2,2',2",2"' '-(1-(phenanthren-9-yl)ethane-1,1,2,2-tetrayl)tetrakis(4,4,5,5-tetramethyl-1,3,2-dioxaborolane) (3n)

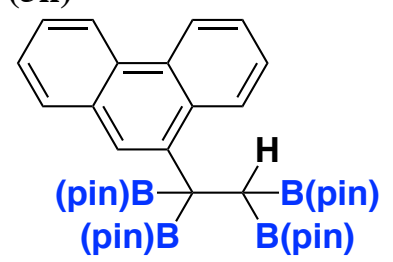

Using the Typical Procedure A, the titled compound was obtained as a white powder in $71 \%$ yield (276 mg, including $9 \mathrm{wt} \%$ hexane, $0.50 \mathrm{mmol} \mathrm{scal})$. 
${ }^{1} \mathrm{H}$ NMR $\left(\mathrm{CDCl}_{3}, 500 \mathrm{MHz}\right): 8.64(\mathrm{~d}, 1 \mathrm{H}, J=5.0 \mathrm{~Hz}), 8.56(\mathrm{~d}, 1 \mathrm{H}, J=5.0 \mathrm{~Hz}), 8.34(\mathrm{~d}, 1 \mathrm{H}, J=5.0 \mathrm{~Hz}), 7.76(\mathrm{~d}$, $1 \mathrm{H}, J=4.0 \mathrm{~Hz}), 7.70(\mathrm{~m}, 1 \mathrm{H}), 7.49-7.43(\mathrm{~m}, 4 \mathrm{H}), 1.36(\mathrm{~s}, 1 \mathrm{H}), 1.27(\mathrm{~s}, 12 \mathrm{H}), 1.23(\mathrm{~s}, 12 \mathrm{H}), 1.19(\mathrm{~s}, 12 \mathrm{H}), 1.12$ (s, 12H). ${ }^{13} \mathrm{C} \mathrm{NMR}\left(\mathrm{CDCl}_{3}, 125 \mathrm{MHz}\right): 132.5,132.4,131.0,129.3,128.3,128.2,126.2,125.7,125.0,124.9$, $124.8,124.4,122.4,122.0,83.3,82.5,25.2,25.0,24.9,24.8,24.7,24.6$. The carbon directly attached to the boron atom was not detected, likely due to quadropolar relaxation. ${ }^{11} \mathrm{~B} \mathrm{NMR}\left(\mathrm{CDCl}_{3}, 160 \mathrm{MHz}\right): 33.9$, 30.6. mp: 207.3-209. $3^{\circ} \mathrm{C}$ (recrystallized from hexane).

$\mathrm{C}_{40} \mathrm{H}_{58} \mathrm{~B}_{4} \mathrm{O}_{8}$. HRMS (pos. ESI) $m / z$ : calcd for $[\mathrm{M}+\mathrm{Na}]^{+} 733.4402$, found 733.4396 .

ATR-FTIR (neat) $v: 2978,1325,1212,1138,970,889,849,772,757,727,668,593,556,481,462,439 \mathrm{~cm}^{-1}$.

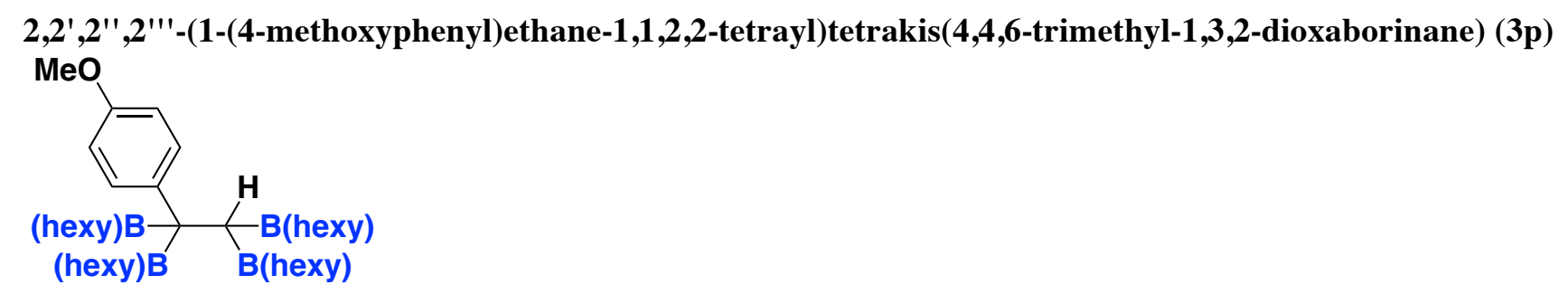

Using the Typical Procedure A, the titled compound was obtained as a colorless amorphous in 34\% yield (54.2 $\mathrm{mg})$. This compound is not stable on silica gel.

${ }^{1} \mathrm{H}$ NMR $\left(\mathrm{CDCl}_{3}, 500 \mathrm{MHz}\right)$ : 7.36-7.31 (m, 2H), 6.67-6.65 (m, 2H), 4.15-3.95 (m, 4H), 3.74 (s, 3H), 1.60-1.58 $(\mathrm{m}, 4 \mathrm{H}), 1.53-1.41(\mathrm{~m}, 4 \mathrm{H}), 1.34-1.12(\mathrm{~m}, 36 \mathrm{H}), 1.34-1.12(\mathrm{~m}, 1 \mathrm{H}) .{ }^{13} \mathrm{C} \mathrm{NMR}\left(\mathrm{CDCl}_{3}, 125 \mathrm{MHz}\right): 129.7,129.4$, $111.8,111.6,69.6,69.5,64.0,63.9,63.7,55.1,55.0,45.9,45.8,45.7,45.6,31.7,31.6,31.5,31.3,28.1,28.0$, $27.9,23.7,23.6,23.3,23.2$. The carbon directly attached to the boron atom was not detected, likely due to quadropolar relaxation. ${ }^{11} \mathrm{~B} \mathrm{NMR}\left(\mathrm{CDCl}_{3}, 160 \mathrm{MHz}\right): 30.0$.

$\mathrm{C}_{27} \mathrm{H}_{45} \mathrm{~B}_{3} \mathrm{O}_{7}$. HRMS (pos. ESI) $\mathrm{m} / z$ : calcd for $[\mathrm{M}+\mathrm{Na}]^{+} 663.4194$, found 663.4200 .

ATR-FTIR (neat) $v: 2967,2352,1507,1378,1294,1240,1203,1161,1046,831,768,707,554,521,468,421$ $\mathrm{cm}^{-1}$.

\section{Typical Procedure B:}

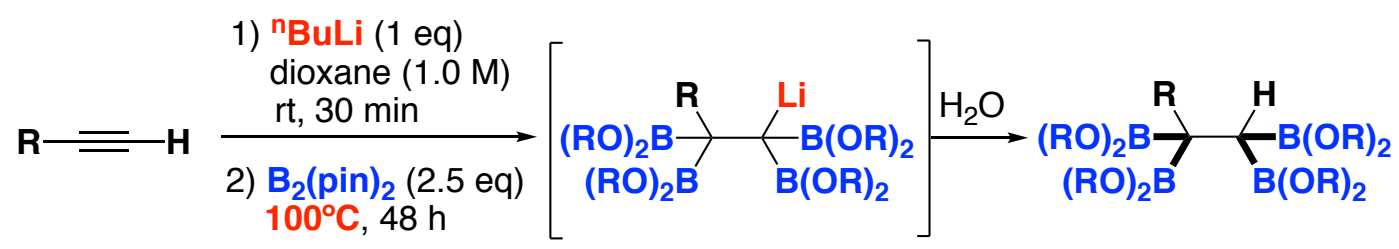

Terminal alkyne $1(0.50 \mathrm{mmol})$ was charged in a dried Schlenk tube, and dissolved in dry dioxane $(0.50 \mathrm{~mL}, 1.0$ $\mathrm{M})$. To the mixture was added ${ }^{\mathrm{n}} \mathrm{BuLi}\left(0.18 \mathrm{~mL}, 0.50 \mathrm{mmol} ; 2.60 \mathrm{M}\right.$ in hexane solution) at $0^{\circ} \mathrm{C}$, and the solution was stirred for $15 \mathrm{~min}$. To the mixture was successively added diboron $(1.25 \mathrm{mmol})$ at room temperature. After the reaction tube was sealed and heated for 48 hours at $100^{\circ} \mathrm{C}$. The reaction was quenched by $\mathrm{H}_{2} \mathrm{O}(1 \mathrm{~mL})$, and then extracted with AcOEt $(15 \mathrm{~mL} \times 3)$. The AcOEt layers were combined and dried over $\mathrm{MgSO}_{4}$, and the solvent was removed under reduced pressure. The residue was purified by silica gel column chromatography using hexane/AcOEt as eluent to give the 1,1,2,2-tetrakis(boronate) compound (3).

\section{2,2',2"',2"'-(octane-1,1,2,2-tetrayl)tetrakis(4,4,5,5-tetramethyl-1,3,2-dioxaborolane) (3q)}




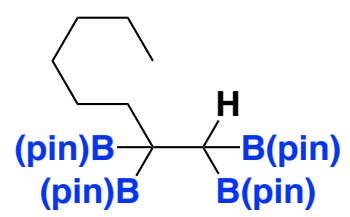

Using the Typical Procedure B, the titled compound was obtained as a white powder in 57\% yield (176 mg). ${ }^{1} \mathrm{H}$ NMR $\left(\mathrm{CDCl}_{3}, 500 \mathrm{MHz}\right): 1.60-1.56(\mathrm{~m}, 2 \mathrm{H}), 1.30-1.26(\mathrm{~m}, 2 \mathrm{H}), 1.26-1.23(\mathrm{~m}, 6 \mathrm{H}), 1.23$ (bs, $\left.12 \mathrm{H}\right), 1.21$ (bs, $12 \mathrm{H}), 1.19(\mathrm{~s}, 12 \mathrm{H}), 0.94(\mathrm{~s}, 1 \mathrm{H}), 0.83(\mathrm{t}, 3 \mathrm{H}, J=8.5 \mathrm{~Hz}) .{ }^{13} \mathrm{C} \mathrm{NMR}\left(\mathrm{CDCl}_{3}, 125 \mathrm{MHz}\right): 82.4,82.3,33.5,31.9$, $30.3,26.9,25.0,24.9,24.7,24.6,24.5,22.7,14.1$. The carbon directly attached to the boron atom was not detected, likely due to quadropolar relaxation. ${ }^{11} \mathrm{~B} \mathrm{NMR}\left(\mathrm{CDCl}_{3}, 160 \mathrm{MHz}\right)$ : 34.6. $\mathrm{mp}: 67.4-71.3^{\circ} \mathrm{C}$ (recrystallized from hexane).

$\mathrm{C}_{32} \mathrm{H}_{62} \mathrm{~B}_{4} \mathrm{O}_{8}$. HRMS (pos. ESI) $\mathrm{m} / \mathrm{z}$ : calcd for $[\mathrm{M}+\mathrm{Na}]^{+} 641.4714$, found 641.4730 .

ATR-FTIR (neat) $v:$ 2973, 2361, 1306, 1263, 1213, 1138, 970, 848, 752, 700, 668, 578, 492, 466, 456, $432 \mathrm{~cm}^{-1}$.

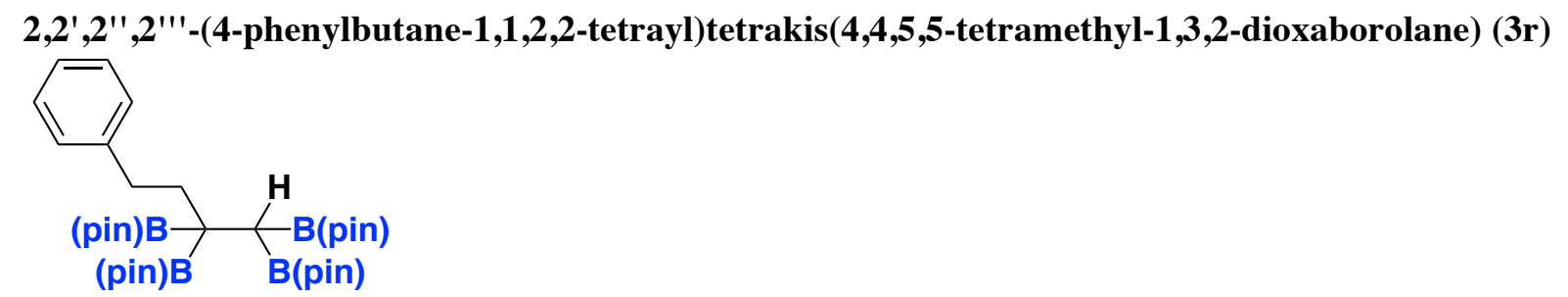

Using the Typical Procedure B, the titled compound was obtained as a white powder in $52 \%$ yield (165.5 mg).

${ }^{1} \mathrm{H}$ NMR $\left(\mathrm{CDCl}_{3}, 500 \mathrm{MHz}\right)$ : 7.22-7.18 (m, 4H), 7.12-7.09 (m, 1H), 2.65-2.61 (m, 2H), 1.91-1.87 (m, 2H), 1.22 $(\mathrm{s}, 24 \mathrm{H}), 1.21(\mathrm{~s}, 12 \mathrm{H}), 1.20(\mathrm{~s}, 12 \mathrm{H}), 1.06(\mathrm{~s}, 1 \mathrm{H}) .{ }^{13} \mathrm{C} \mathrm{NMR}\left(\mathrm{CDCl}_{3}, 125 \mathrm{MHz}\right): 144.9,128.7,127.9,125.0$, $82.6,82.4,36.2,34.1,25.0,24.9,24.8,24.7$. The carbon directly attached to the boron atom was not detected, likely due to quadropolar relaxation. ${ }^{11} \mathrm{~B} \mathrm{NMR}\left(\mathrm{CDCl}_{3}, 160 \mathrm{MHz}\right)$ : $31.1 . \mathrm{mp}: 108.8-110.4^{\circ} \mathrm{C}$ (recrystallized from hexane).

$\mathrm{C}_{34} \mathrm{H}_{58} \mathrm{~B}_{4} \mathrm{O}_{8}$. HRMS (pos. ESI) $\mathrm{m} / \mathrm{z}$ : calcd for $[\mathrm{M}+\mathrm{Na}]^{+} 661.4401$, found 661.4389 .

ATR-FTIR (neat) $v: 2973,2361,1314,1262,1212,1137,1024,970,848,752,700,666,500,457,419 \mathrm{~cm}^{-1}$.

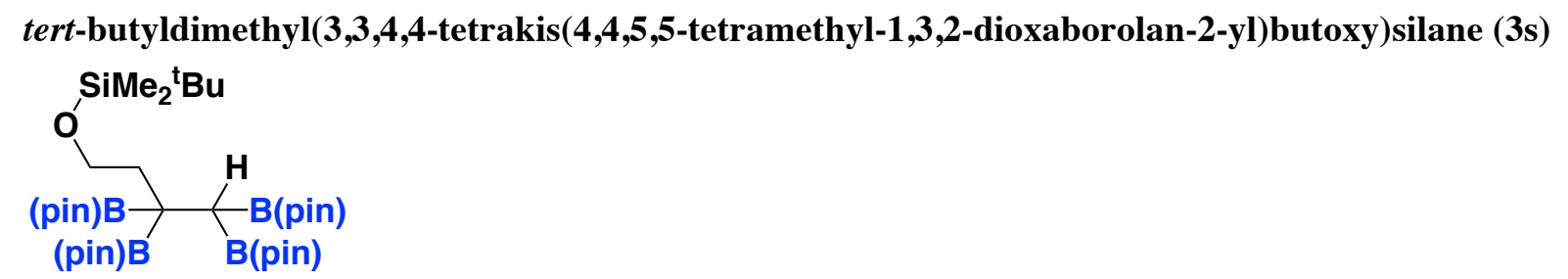

Using the Typical Procedure B, the titled compound was obtained as a white powder in $40 \%$ yield (138 mg).

${ }^{1} \mathrm{H} \mathrm{NMR}\left(\mathrm{CDCl}_{3}, 500 \mathrm{MHz}\right): 3.71(\mathrm{t}, 2 \mathrm{H}, J=13.8 \mathrm{~Hz}), 1.88(\mathrm{t}, 2 \mathrm{H}, J=13.8 \mathrm{~Hz}), 1.19(\mathrm{~s}, 48 \mathrm{H}), 0.93(\mathrm{~s}, 1 \mathrm{H})$, 0.88 (s, 9H), 0.04 (s, 6H). ${ }^{13} \mathrm{C} \mathrm{NMR}\left(\mathrm{CDCl}_{3}, 125 \mathrm{MHz}\right)$ : 82.6, 82.4, 63.2, 36.2, 26.2, 24.9, 18.5, -5.0. The carbon directly attached to the boron atom was not detected, likely due to quadropolar relaxation. ${ }^{11} \mathrm{~B} \mathrm{NMR}\left(\mathrm{CDCl}_{3}, 160\right.$ $\mathrm{MHz}$ ): $34.7,28.5 . \mathrm{mp}: 163.0-165.3^{\circ} \mathrm{C}$ (recrystallized from hexane).

$\mathrm{C}_{34} \mathrm{H}_{68} \mathrm{~B}_{4} \mathrm{O}_{9} \mathrm{Si}$. HRMS (pos. ESI) $\mathrm{m} / z$ : calcd for $[\mathrm{M}+\mathrm{Na}]^{+}$715.4902, found 715.4888 .

ATR-FTIR (neat) $v: 2975,2363,1309,1264,1213,1138,969,847,773,670,562,478,467,445,418 \mathrm{~cm}^{-1}$. 


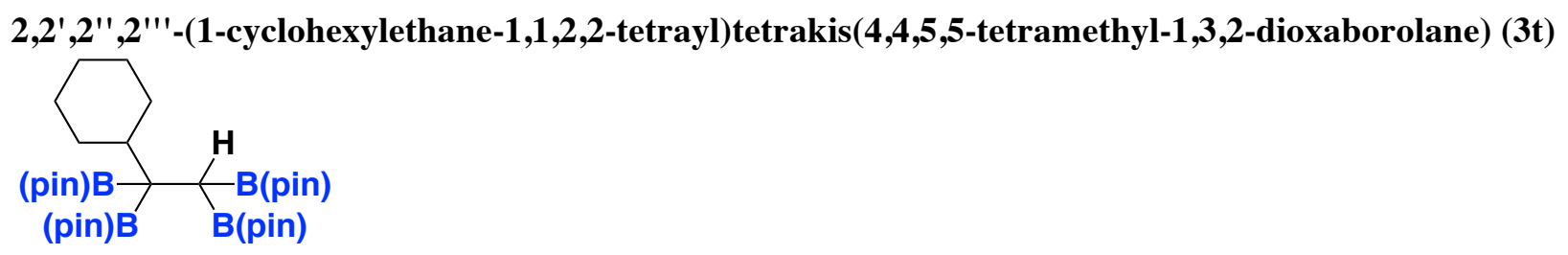

Using the Typical Procedure B, the titled compound was obtained as a white powder in 54\% yield (166 mg).

${ }^{1} \mathrm{H}$ NMR $\left(\mathrm{CDCl}_{3}, 500 \mathrm{MHz}\right): 1.94(\mathrm{~d}, 2 \mathrm{H}, J=11.9 \mathrm{~Hz}), 1.70-1.56(\mathrm{~m}, 4 \mathrm{H}), 1.26-1.24(\mathrm{~m}, 1 \mathrm{H}), 1.21(\mathrm{~s}, 12 \mathrm{H})$, 1.19 (s, 12H), 1.10-0.97 (m, 4H), 0.95 (s, 1H). ${ }^{13} \mathrm{C} \mathrm{NMR}\left(\mathrm{CDCl}_{3}, 125 \mathrm{MHz}\right): 82.3,82.2$, 41.6, 31.4, 27.5, 27.1, $25.3,25.0,24.8,24.7$. The carbon directly attached to the boron atom was not detected, likely due to quadropolar relaxation. ${ }^{11} \mathrm{~B}$ NMR $\left(\mathrm{CDCl}_{3}, 160 \mathrm{MHz}\right)$ : 35.3, 32.2. $\mathrm{mp}$ : 199.0-200.1 ${ }^{\circ} \mathrm{C}$ (recrystallized from hexane).

$\mathrm{C}_{32} \mathrm{H}_{62} \mathrm{~B}_{4} \mathrm{O}_{8}$. HRMS (pos. ESI) $\mathrm{m} / z$ : calcd for $[\mathrm{M}+\mathrm{Na}]^{+} 639.4558$, found 639.4562 .

ATR-FTIR (neat) $v: 2973,2923,2358,2181,1328,1264,1230,1135,972,847,667,564,532,511,494,483$, $474,461,443,432 \mathrm{~cm}^{-1}$.

\section{1,1,2,2-tetrakis(4,4,5,5-tetramethyl-1,3,2-dioxaborolan-2-yl)cyclopentane (3u)}

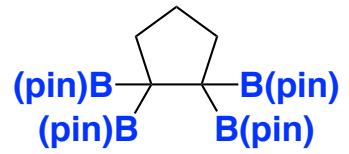

Using the Typical Procedure A, the titled compound was obtained as a white powder in $45 \%$ yield (149 $\mathrm{mg})$. ${ }^{1} \mathrm{H}$ NMR $\left(\mathrm{CDCl}_{3}, 500 \mathrm{MHz}\right): 1.85(\mathrm{t}, 4 \mathrm{H}, J=7.4 \mathrm{~Hz}), 1.57-1.53(\mathrm{~m}, 4 \mathrm{H}), 1.20(\mathrm{~s}, 24 \mathrm{H}), 1.20(\mathrm{~s}, 24 \mathrm{H}) .{ }^{13} \mathrm{C}$ NMR $\left(\mathrm{CDCl}_{3}, 125 \mathrm{MHz}\right): 82.4,31.2,25.2,24.8,24.6$. The carbon directly attached to the boron atom was not detected, likely due to quadropolar relaxation. ${ }^{11} \mathrm{~B} \mathrm{NMR}\left(\mathrm{CDCl}_{3}, 160 \mathrm{MHz}\right)$ : $34.7 . \mathrm{mp}$ : $116.1-118.1^{\circ} \mathrm{C}$ (recrystallized from hexane).

$\mathrm{C}_{29} \mathrm{H}_{54} \mathrm{~B}_{4} \mathrm{O}_{8}$. HRMS (pos. ESI) $m / z$ : calcd for $[\mathrm{M}+\mathrm{Na}]^{+}$597.4088, found 597.4100 .

ATR-FTIR (neat) $v: 2974,2358,1508,1377,1285,1242,1161,833,669,547,532,514,478,466 \mathrm{~cm}^{-1}$.

\subsection{In Situ Electrophilic Trapping of the Intermediates (Scheme 2)}

\section{Typical Procedure C:}

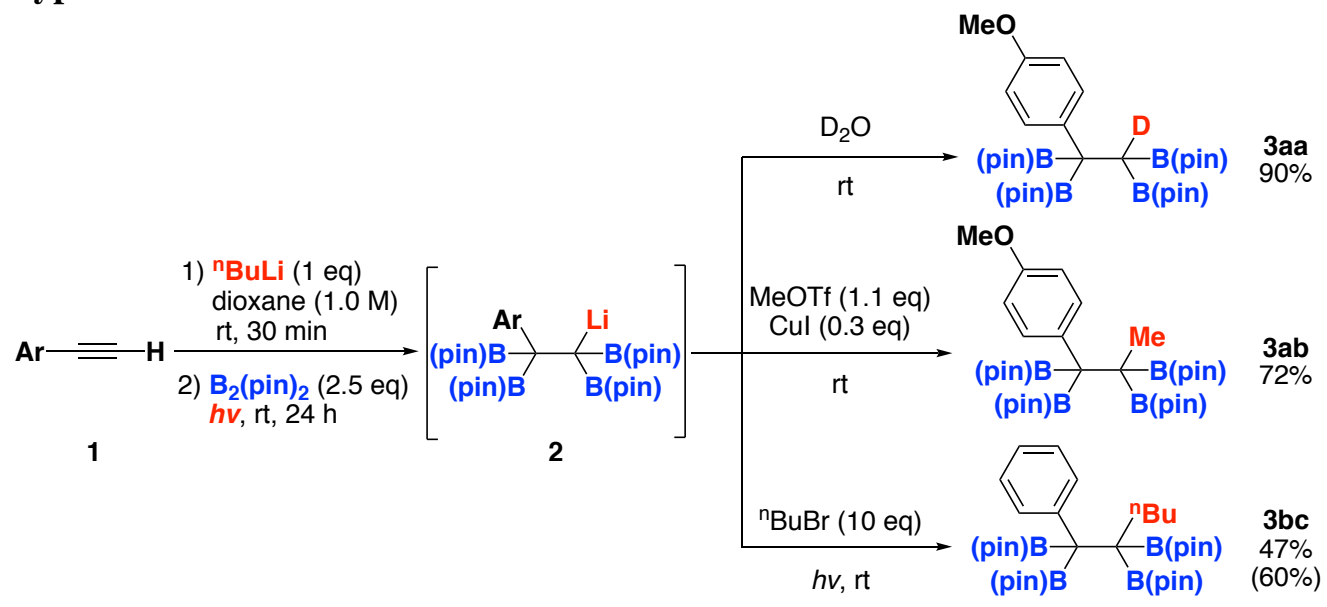

Terminal alkyne $1(0.25 \mathrm{mmol})$ was charged in a dried Schlenk tube, and dissolved in dry dioxane $(0.25 \mathrm{~mL}, 1.0$ 
M). To the mixture was added ${ }^{n} \mathrm{BuLi}\left(0.09 \mathrm{~mL}, 0.25 \mathrm{mmol} ; 2.60 \mathrm{M}\right.$ in hexane solution) at $0^{\circ} \mathrm{C}$, and the solution was stirred for $15 \mathrm{~min}$. To the mixture was successively added diboron $(0.625 \mathrm{mmol})$ at room temperature. After the reaction tube was sealed and irradiated for 24 hours with a $430 \mathrm{~mW} / \mathrm{cm}^{-1}$ medium-pressure $\mathrm{Hg}$ lamp equipped with a cooling fan. The reaction was quenched by electrophiles and stirred for 24 hours. Then the reaction was quenched by $\mathrm{H}_{2} \mathrm{O}$ and extracted with AcOEt $(15 \mathrm{~mL} \times 3)$. The AcOEt layers were combined and dried over $\mathrm{MgSO}_{4}$, and the solvent was removed under reduced pressure. The residue was purified by silica gel column chromatography using hexane/AcOEt as eluent to give the 1,1,2,2-tetrakis(boronate) compound (3).

\section{2,2',2",2"'-(1-(4-methoxyphenyl)propane-1,1,2,2-tetrayl)tetrakis(4,4,5,5-tetramethyl-1,3,2-dioxaborolane)}

(3ab)

$\mathrm{MeO}$

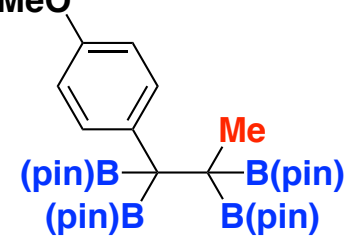

Using the Typical Procedure C, the titled compound was obtained as a colorless oil in $72 \%$ yield (117 mg). ${ }^{1} \mathrm{H} \mathrm{NMR}\left(\mathrm{CDCl}_{3}, 500 \mathrm{MHz}\right): 7.43(\mathrm{~d}, 2 \mathrm{H}, J=8.5 \mathrm{~Hz}), 6.69$ (d, 2H, $\left.J=8.5 \mathrm{~Hz}\right), 3.74(\mathrm{~s}, 3 \mathrm{H}), 1.27$ (bs, 24H), 1.21 (bs, 24H), 1.10 (s, 3H). ${ }^{13} \mathrm{C} \mathrm{NMR}\left(\mathrm{CDCl}_{3}, 125 \mathrm{MHz}\right): 156.8,134.8,133.5,112.4,82.6,55.1,25.2,24.8$, 15.9. The carbon directly attached to the boron atom was not detected, likely due to quadropolar relaxation. ${ }^{11} \mathrm{~B}$ $\operatorname{NMR}\left(\mathrm{CDCl}_{3}, 160 \mathrm{MHz}\right): 34.2$.

$\mathrm{C}_{34} \mathrm{H}_{58} \mathrm{~B}_{4} \mathrm{O}_{9}$. HRMS (pos. ESI) $m / z$ : calcd for $[\mathrm{M}+\mathrm{Na}]^{+}$677.4351, found 677.4334 . ATR-FTIR (neat) $v: 2977,1469,1329,1251,1214,1133,969,848,725,666,579,454,439 \mathrm{~cm}^{-1}$.

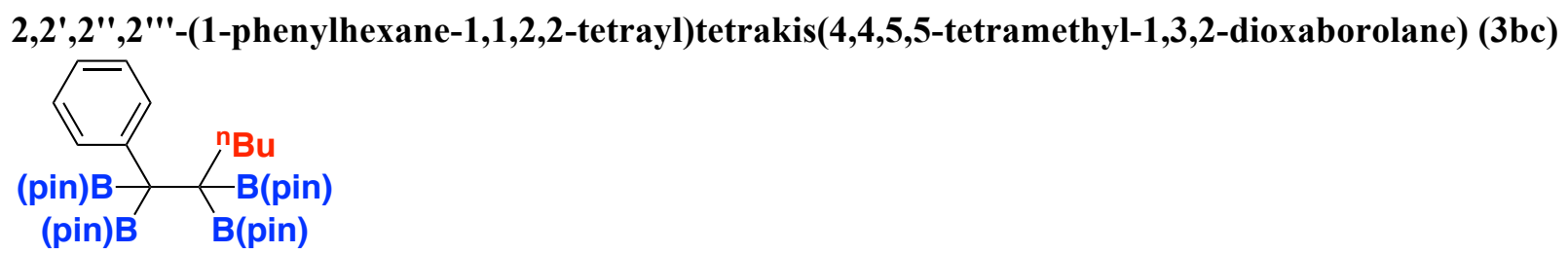

Using the Typical Procedure C, the titled compound was obtained as a white solid in $47 \%$ yield $(78.1 \mathrm{mg})$. ${ }^{1} \mathrm{H} \mathrm{NMR}\left(\mathrm{CDCl}_{3}, 500 \mathrm{MHz}\right): 7.47$ (t, 2H, $\left.J=9.0 \mathrm{~Hz}\right), 7.10(\mathrm{t}, 1 \mathrm{H}, J=9.0 \mathrm{~Hz}), 7.09-7.03(\mathrm{~m}, 2 \mathrm{H}), 1.28(\mathrm{~s}, 24 \mathrm{H})$, 1.29-1.22 (m, 24H), 1.29-1.08 (m, 6H), $0.80(\mathrm{t}, 3 \mathrm{H}, J=9.0 \mathrm{~Hz}) \cdot{ }^{13} \mathrm{C}$ NMR $\left(\mathrm{CDCl}_{3}, 125 \mathrm{MHz}\right): 133.2,132.5$, $127.9,126.5,82.4,82.3,30.9,30.3,25.1,25.0,24.0,14.2$. The carbon directly attached to the boron atom was not detected, likely due to quadropolar relaxation. ${ }^{11} \mathrm{~B} \mathrm{NMR}\left(\mathrm{CDCl}_{3}, 160 \mathrm{MHz}\right)$ : 34.1. mp: $>250^{\circ} \mathrm{C}$ (recrystallized from hexane).

$\mathrm{C}_{36} \mathrm{H}_{62} \mathrm{~B}_{4} \mathrm{O}_{8}$. HRMS (pos. ESI) $m / z$ : calcd for $[\mathrm{M}+\mathrm{Na}]^{+} 689.4715$, found 689.4702 .

ATR-FTIR (neat) $v: 2978,1509,1461,1318,1247,1133,1033,962,844,731,677,577,449,439 \mathrm{~cm}^{-1}$.

\subsection{Transformations of the 1,1,2,2-Tetraborylated Product (3a) (Scheme 5)}

\section{Typical Procedure D:}




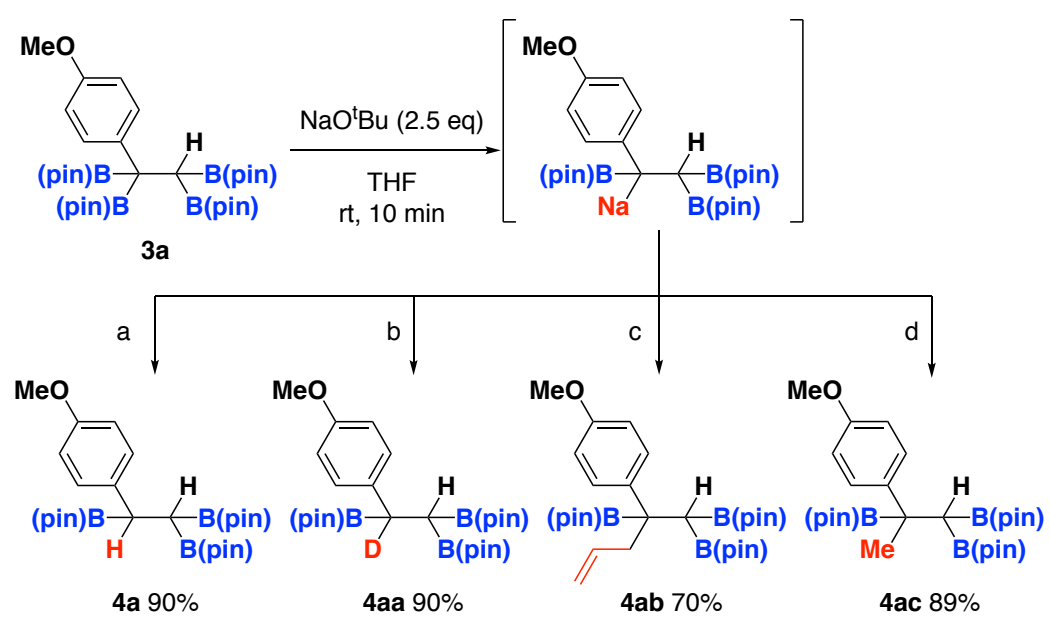

2,2',2",2'"-(1-(4-methoxyphenyl)ethane-1,1,2,2-tetrayl)tetrakis(4,4,5,5-tetramethyl-1,3,2-dioxaborolane)

(3a, $64.0 \mathrm{mg}, 0.10 \mathrm{mmol})$ was charged in a dried Schlenk tube, and dissolved in dry THF $(0.5 \mathrm{~mL})$. To the mixture was added $\mathrm{NaO}^{\mathrm{t}} \mathrm{Bu}(15.2 \mathrm{mg}, 0.20 \mathrm{mmol})$ at room temperature, and the solution was stirred for $10 \mathrm{~min}$ at room temperature. To the mixture were successively added electrophiles $(0.20 \mathrm{mmol})$ at room temperature and the mixture was stirred for $18 \mathrm{~h}$. The reaction was quenched by $\mathrm{H}_{2} \mathrm{O}(2 \mathrm{~mL})$, and then extracted with AcOEt $(10 \mathrm{~mL}$ $\times 3$ ). The AcOEt layers were combined and dried over $\mathrm{MgSO}_{4}$, and the solvent was removed under reduced pressure. The residue was purified by silica gel column chromatography using hexane/AcOEt as eluent to give the titled compound.

\section{2,2',2''-(2-(4-methoxyphenyl)ethane-1,1,2-triyl)tris(4,4,5,5-tetramethyl-1,3,2-dioxaborolane) (4a) $\mathrm{MeO}$

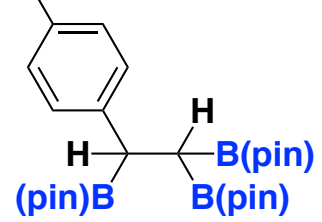

Using the Typical Procedure D, the titled compound was obtained as a white powder in $90 \%$ yield (46.1 mg). ${ }^{1} \mathrm{H} \mathrm{NMR}\left(\mathrm{CDCl}_{3}, 500 \mathrm{MHz}\right): 7.12(\mathrm{~d}, 2 \mathrm{H}, J=8.5 \mathrm{~Hz}), 6.73(\mathrm{~d}, 2 \mathrm{H}, J=8.5 \mathrm{~Hz}), 3.74(\mathrm{~s}, 3 \mathrm{H}), 2.61(\mathrm{~d}, 1 \mathrm{H}, J=$ $13.0 \mathrm{~Hz}), 1.37(\mathrm{~d}, 1 \mathrm{H}, J=13.0 \mathrm{~Hz}), 1.23(\mathrm{~s}, 6 \mathrm{H}), 1.22(\mathrm{~s}, 6 \mathrm{H}), 1.15(\mathrm{~s}, 12 \mathrm{H}), 1.13(\mathrm{~s}, 12 \mathrm{H}), 0.96(\mathrm{~s}, 6 \mathrm{H}), 0.95(\mathrm{~s}$, $6 \mathrm{H}) .{ }^{13} \mathrm{C} \mathrm{NMR}\left(\mathrm{CDCl}_{3}, 125 \mathrm{MHz}\right)$ : 157.0, 137.5, 129.4, 113.3, 83.0, 82.9, 82.6, 55.2, 24.9, 24.7, 24.5, 24.4, 24.3. The carbon directly attached to the boron atom was not detected, likely due to quadropolar relaxation. ${ }^{11} \mathrm{~B}$ NMR $\left(\mathrm{CDCl}_{3}, 160 \mathrm{MHz}\right): 33.7$.

$\mathrm{C}_{27} \mathrm{H}_{45} \mathrm{~B}_{3} \mathrm{O}_{7}$. HRMS (pos. ESI) $m / z$ : calcd for $[\mathrm{M}+\mathrm{Na}]^{+}$537.3342, found 537.3332. ATR-FTIR (neat) $v: 2977,2360,2334,1300,1292,1137,1031,970,847,561,475,460,420 \mathrm{~cm}^{-1}$.

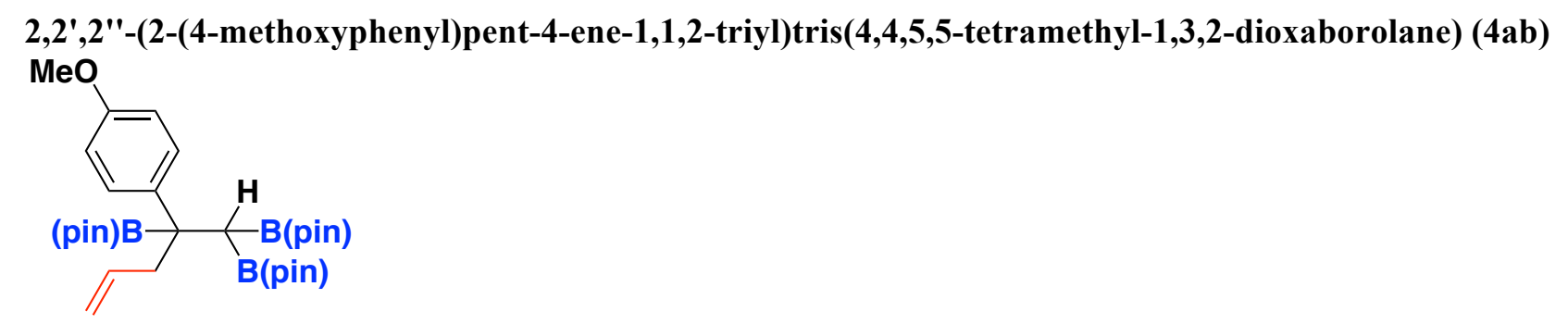


Using the Typical Procedure D, the titled compound was obtained as a colorless gum in $70 \%$ yield (38.7 $\mathrm{mg})$. ${ }^{1} \mathrm{H}$ NMR $\left(\mathrm{CDCl}_{3}, 500 \mathrm{MHz}\right): 7.35(\mathrm{~d}, 2 \mathrm{H}, J=8.0 \mathrm{~Hz}), 6.74(\mathrm{~d}, 2 \mathrm{H}, J=8.0 \mathrm{~Hz}), 5.67(\mathrm{ddt}, 1 \mathrm{H}, J=16.5,13.0,7.5$ $\mathrm{Hz}), 4.98-4.94(\mathrm{~m}, 1 \mathrm{H}), 4.87-4.84(\mathrm{~m}, 1 \mathrm{H}), 3.75(\mathrm{~s}, 3 \mathrm{H}), 2.80(\mathrm{~d}, 2 \mathrm{H}, J=7.5 \mathrm{~Hz}), 1.30(\mathrm{~s}, 1 \mathrm{H}), 1.25(\mathrm{~s}, 6 \mathrm{H}), 1.23$ $(\mathrm{s}, 6 \mathrm{H}), 1.15(\mathrm{~s}, 12 \mathrm{H}), 1.12(\mathrm{~s}, 6 \mathrm{H}), 1.10(\mathrm{~s}, 6 \mathrm{H}) .{ }^{13} \mathrm{C} \mathrm{NMR}\left(\mathrm{CDCl}_{3}, 125 \mathrm{MHz}\right): 156.7,139.4,137.6,129.4,115.9$, 112.6, 83.2, 82.5, 82.4, 55.1, 42.4, 24.8, 24.8. The carbon directly attached to the boron atom was not detected, likely due to quadropolar relaxation. ${ }^{11} \mathrm{~B} \mathrm{NMR}\left(\mathrm{CDCl}_{3}, 160 \mathrm{MHz}\right): 33.0$.

$\mathrm{C}_{30} \mathrm{H}_{49} \mathrm{~B}_{3} \mathrm{O}_{7}$. HRMS (pos. ESI) $m / z$ : calcd for $[\mathrm{M}+\mathrm{Na}]^{+}$577.3655, found 577.3661 .

ATR-FTIR (neat) $v: 2977,1509,1457,1318,1247,1214,1140,1038,969,850,735,675,578,453,439 \mathrm{~cm}^{-1}$.

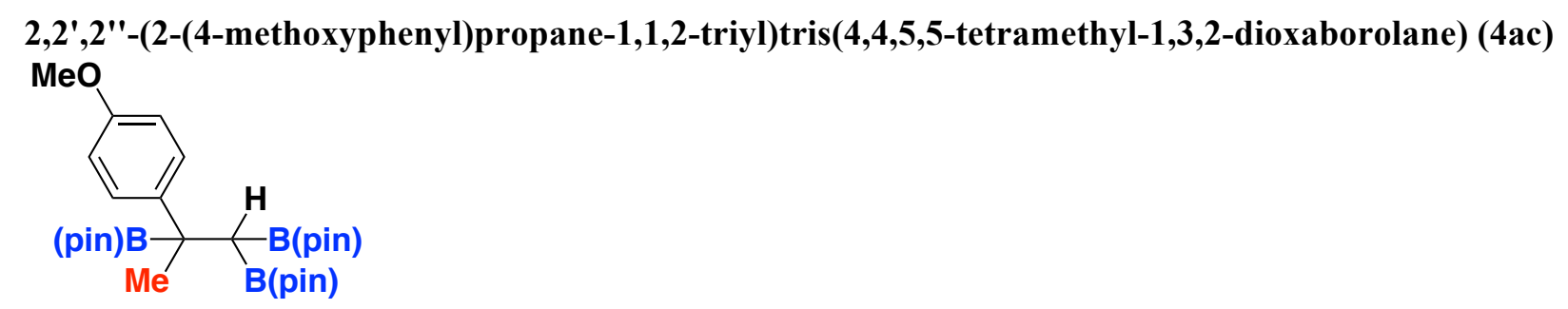

Using the Typical Procedure D, the titled compound was obtained as a colorless gum in $89 \%$ yield (93.8 mg, $0.20 \mathrm{mmol}$ scale).

${ }^{1} \mathrm{H}$ NMR $\left(\mathrm{CDCl}_{3}, 500 \mathrm{MHz}\right): 7.30$ (d, 2H, $\left.J=7.0 \mathrm{~Hz}\right), 6.75(\mathrm{~d}, 2 \mathrm{H}, J=7.0 \mathrm{~Hz}), 3.75(\mathrm{~s}, 3 \mathrm{H}), 1.64(\mathrm{~s}, 1 \mathrm{H}), 1.59$ (s, $3 \mathrm{H}), 1.22(\mathrm{~s}, 6 \mathrm{H}), 1.20(\mathrm{~s}, 6 \mathrm{H}), 1.13(\mathrm{~s}, 6 \mathrm{H}), 1.12(\mathrm{~s}, 6 \mathrm{H}), 0.99(\mathrm{~s}, 6 \mathrm{H}), 0.96(\mathrm{~s}, 6 \mathrm{H}),{ }^{13} \mathrm{C} \mathrm{NMR}\left(\mathrm{CDCl}_{3}, 125\right.$ $\mathrm{MHz}): 156.7,141.2,128.0,112.9,83.0,82.7,82.2,55.2,24.9,24.8,24.7,24.5,24.3,24.2,20.3$. The carbon directly attached to the boron atom was not detected, likely due to quadropolar relaxation. ${ }^{11} \mathrm{~B} \mathrm{NMR}\left(\mathrm{CDCl}_{3}, 160\right.$ $\mathrm{MHz})$ : 35.2, 27.9.

$\mathrm{C}_{28} \mathrm{H}_{47} \mathrm{~B}_{3} \mathrm{O}_{7}$. HRMS (pos. ESI) $m / z$ : calcd for $[\mathrm{M}+\mathrm{Na}]^{+}$551.3499, found 551.3509.

ATR-FTIR (neat) $v: 2977,1509,1457,1248,1140,1037,969,850,669,530,505,466,431 \mathrm{~cm}^{-1}$. 


\section{Computational Details}

All calculations were carried with the Gaussian 16 program package. ${ }^{1}$ The molecular structures and harmonic vibrational frequencies were obtained using the hybrid density functional method based on the unrestricted B3LYP ${ }^{2}$ method. The $6-31+\mathrm{G}^{*}$ basis set was used for all atoms. Geometry optimization and vibrational analysis were performed at the same level. All stationary points were optimized without any symmetry assumptions, and characterized by normal coordinate analysis at the same level of theory (number of imaginary frequencies, NIMAG, 0 for minima and 1 for TSs). The intrinsic reaction coordinate (IRC) method was used to track minimum energy paths from transition structures to the corresponding local minima. ${ }^{3}$ Excitation wavelengths and oscillator strengths were obtained at the density functional level using time-dependent perturbation theory (TD-DFT) approach.

\begin{tabular}{|c|c|c|c|c|c|}
\hline \multicolumn{6}{|c|}{$\begin{array}{l}\text { Reactants } \\
\text { bis(neopentylglycolato)diboron }\end{array}$} \\
\hline Center & Atomic & Atomic & ---------- & rdinates & gstroms ) \\
\hline Number & Number & Type & $\mathrm{x}$ & $\mathrm{Y}$ & $\mathrm{Z}$ \\
\hline 1 & 6 & 0 & -0.080682 & 0769072 & 3003876 \\
\hline 2 & 6 & 0 & 0.080682 & -0.769072 & 3.003876 \\
\hline 3 & 1 & 0 & 0.711389 & 1.284724 & 3.554617 \\
\hline 4 & 1 & 0 & -1.052212 & 1.092909 & 3.392871 \\
\hline 5 & 1 & 0 & 1.052212 & -1.092909 & 3.392871 \\
\hline 6 & 1 & 0 & -0.711389 & -1.284724 & 3.554617 \\
\hline 7 & 5 & 0 & 0.000000 & -0.000000 & 0.851704 \\
\hline 8 & 8 & 0 & 0.000000 & 1.145611 & 1.613137 \\
\hline 9 & 8 & 0 & -0.000000 & -1.145611 & 1.613137 \\
\hline 10 & 6 & 0 & -0.769072 & -0.080682 & -3.003876 \\
\hline 11 & 6 & 0 & 0.769072 & 0.080682 & -3.003876 \\
\hline 12 & 1 & 0 & -1.092909 & -1.052212 & -3.392871 \\
\hline 13 & 1 & 0 & -1.284724 & 0.711389 & -3.554617 \\
\hline 14 & 1 & 0 & 1.284724 & -0.711389 & -3.554617 \\
\hline 15 & 1 & 0 & 1.092909 & 1.052212 & -3.392871 \\
\hline 16 & 5 & 0 & 0.000000 & 0.000000 & -0.851704 \\
\hline 17 & 8 & 0 & 1.145611 & -0.000000 & -1.613137 \\
\hline 18 & 8 & 0 & -1.145611 & 0.000000 & -1.613137 \\
\hline
\end{tabular}

\section{PhCCLi}

Sum of electronic and thermal Free Energies= $\quad-315.276517$ Standard orientation:

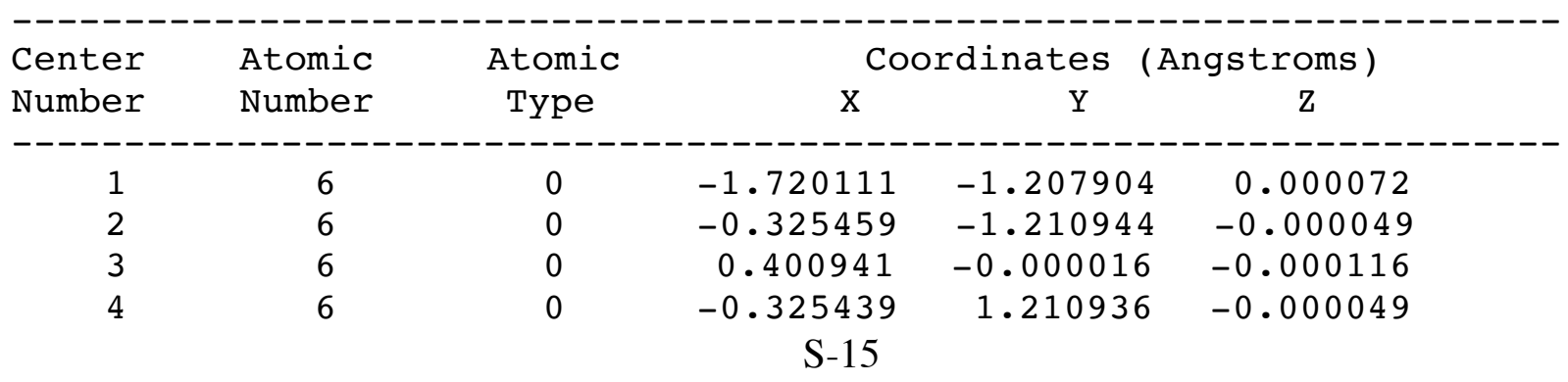




\begin{tabular}{rrrrrr}
5 & 6 & 0 & -1.720083 & 1.207925 & 0.000072 \\
6 & 6 & 0 & -2.425941 & 0.000013 & 0.000132 \\
7 & 1 & 0 & -2.259118 & -2.152653 & 0.000120 \\
8 & 1 & 0 & 0.221387 & -2.149627 & -0.000094 \\
9 & 1 & 0 & 0.221438 & 2.149600 & -0.000094 \\
10 & 1 & 0 & -2.259082 & 2.152678 & 0.000120 \\
11 & 1 & 0 & -3.513124 & 0.000030 & 0.000228 \\
12 & 6 & 0 & 1.830787 & -0.000017 & -0.000241 \\
13 & 6 & 0 & 3.067383 & -0.000014 & -0.000308 \\
14 & 3 & 0 & 4.965346 & 0.000034 & 0.000885 \\
\hline
\end{tabular}

\section{IM1}

Sum of electronic and thermal Free Energies=

$-823.222084$

Standard orientation:

\begin{tabular}{|c|c|c|c|c|c|}
\hline \multirow{2}{*}{$\begin{array}{l}\text { Center } \\
\text { Number }\end{array}$} & \multirow{2}{*}{$\begin{array}{l}\text { Atomic } \\
\text { Number }\end{array}$} & \multirow{2}{*}{$\begin{array}{l}\text { Atomic } \\
\text { Type }\end{array}$} & \multicolumn{3}{|c|}{ Coordinates (Angstroms) } \\
\hline & & & $\mathrm{X}$ & $\mathrm{Y}$ & $\mathrm{Z}$ \\
\hline & & & & & \\
\hline 1 & 6 & 0 & -4.791936 & 0.072169 & -1.235302 \\
\hline 2 & 6 & 0 & -3.430687 & -0.224480 & -1.168887 \\
\hline 3 & 6 & 0 & -2.705386 & -0.018594 & 0.023879 \\
\hline 4 & 6 & 0 & -3.392467 & 0.491968 & 1.146006 \\
\hline 5 & 6 & 0 & -4.753900 & 0.785370 & 1.073410 \\
\hline 6 & 6 & 0 & -5.460844 & 0.578464 & -0.115968 \\
\hline 7 & 1 & 0 & -5.333652 & -0.095127 & -2.163407 \\
\hline 8 & 1 & 0 & -2.913377 & -0.623211 & -2.037208 \\
\hline 9 & 1 & 0 & -2.843549 & 0.651055 & 2.069663 \\
\hline 10 & 1 & 0 & -5.265780 & 1.176427 & 1.949566 \\
\hline 11 & 1 & 0 & -6.522231 & 0.807421 & -0.169346 \\
\hline 12 & 6 & 0 & -1.309566 & -0.319026 & 0.099290 \\
\hline 13 & 6 & 0 & -0.119080 & -0.591396 & 0.190848 \\
\hline 14 & 5 & 0 & 2.207322 & 0.777517 & 0.220981 \\
\hline 15 & 8 & 0 & 1.926258 & -1.651297 & 1.370204 \\
\hline 16 & 8 & 0 & 1.920714 & -1.607103 & -0.992290 \\
\hline 17 & 6 & 0 & 2.189488 & -2.945353 & -0.565328 \\
\hline 18 & 1 & 0 & 1.633511 & -3.658365 & -1.187766 \\
\hline 19 & 1 & 0 & 3.265434 & -3.150492 & -0.663629 \\
\hline 20 & 6 & 0 & 1.746293 & -2.976450 & 0.912792 \\
\hline 21 & 1 & 0 & 0.688500 & -3.277528 & 0.987380 \\
\hline 22 & 1 & 0 & 2.351220 & -3.661139 & 1.518637 \\
\hline 23 & 8 & 0 & 2.391675 & 1.750178 & 1.161636 \\
\hline 24 & 8 & 0 & 2.694973 & 1.244499 & -1.046026 \\
\hline 25 & 6 & 0 & 3.080448 & 2.636970 & -0.922035 \\
\hline 26 & 1 & 0 & 4.054596 & 2.782261 & -1.396592 \\
\hline 27 & 1 & 0 & 2.330441 & 3.248333 & -1.434748 \\
\hline 28 & 6 & 0 & 3.099126 & 2.870418 & 0.601737 \\
\hline 29 & 1 & 0 & 2.591485 & 3.793621 & 0.894000 \\
\hline 30 & 1 & 0 & 4.116280 & 2.879088 & 1.009089 \\
\hline 31 & 5 & 0 & 1.462325 & -0.790713 & 0.294249 \\
\hline 32 & 3 & 0 & 2.373665 & -0.285646 & -2.047545 \\
\hline
\end{tabular}


TS1

Sum of electronic and thermal Free Energies=

$-823.180333$

Standard orientation:

\begin{tabular}{|c|c|c|c|c|c|}
\hline \multirow{2}{*}{$\begin{array}{l}\text { Center } \\
\text { Number }\end{array}$} & \multirow{2}{*}{$\begin{array}{l}\text { Atomic } \\
\text { Number }\end{array}$} & \multirow{2}{*}{$\begin{array}{c}\text { Atomic } \\
\text { Type }\end{array}$} & \multicolumn{3}{|c|}{ Coordinates (Angstroms) } \\
\hline & & & $\mathrm{x}$ & $\mathrm{Y}$ & $\mathrm{z}$ \\
\hline 1 & 6 & 0 & -4.138658 & -0.857154 & -1.089012 \\
\hline 2 & 6 & 0 & -2.746639 & -0.895368 & -1.141745 \\
\hline 3 & 6 & 0 & -1.982170 & -0.765766 & 0.040395 \\
\hline 4 & 6 & 0 & -2.660995 & -0.593517 & 1.267933 \\
\hline 5 & 6 & 0 & -4.054023 & -0.557008 & 1.306794 \\
\hline 6 & 6 & 0 & -4.802422 & -0.686052 & 0.131523 \\
\hline 7 & 1 & 0 & -4.709894 & -0.963566 & -2.008175 \\
\hline 8 & 1 & 0 & -2.234248 & -1.026483 & -2.090311 \\
\hline 9 & 1 & 0 & -2.084971 & -0.498111 & 2.184220 \\
\hline 10 & 1 & 0 & -4.558798 & -0.430589 & 2.261816 \\
\hline 11 & 1 & 0 & -5.888271 & -0.658661 & 0.166668 \\
\hline 12 & 6 & 0 & -0.555829 & -0.775602 & -0.017240 \\
\hline 13 & 6 & 0 & 0.641582 & -1.173566 & -0.036643 \\
\hline 14 & 5 & 0 & 0.760006 & 0.622428 & -0.256783 \\
\hline 15 & 8 & 0 & 2.909350 & -0.464619 & 1.094656 \\
\hline 16 & 8 & 0 & 2.987034 & -0.981750 & -1.202296 \\
\hline 17 & 6 & 0 & 4.311480 & -0.887838 & -0.710100 \\
\hline 18 & 1 & 0 & 4.934739 & -1.660039 & -1.175879 \\
\hline 19 & 1 & 0 & 4.742456 & 0.099696 & -0.944332 \\
\hline 20 & 6 & 0 & 4.175763 & -1.074222 & 0.806836 \\
\hline 21 & 1 & 0 & 4.133324 & -2.137612 & 1.079358 \\
\hline 22 & 1 & 0 & 4.964012 & -0.582146 & 1.387203 \\
\hline 23 & 8 & 0 & 0.620771 & 1.510845 & 0.949828 \\
\hline 24 & 8 & 0 & 0.436324 & 1.409496 & -1.390802 \\
\hline 25 & 6 & 0 & -0.436849 & 2.438860 & -0.934261 \\
\hline 26 & 1 & 0 & -0.358310 & 3.304040 & -1.600626 \\
\hline 27 & 1 & 0 & -1.477086 & 2.082946 & -0.931077 \\
\hline 28 & 6 & 0 & 0.036796 & 2.748678 & 0.498260 \\
\hline 29 & 1 & 0 & -0.780532 & 3.035386 & 1.168822 \\
\hline 30 & 1 & 0 & 0.807087 & 3.531321 & 0.510382 \\
\hline 31 & 5 & 0 & 2.081140 & -0.602297 & -0.165461 \\
\hline 32 & 3 & 0 & 1.982905 & 0.827574 & 1.918545 \\
\hline
\end{tabular}

IM2

Sum of electronic and thermal Free Energies=

$-823.235074$

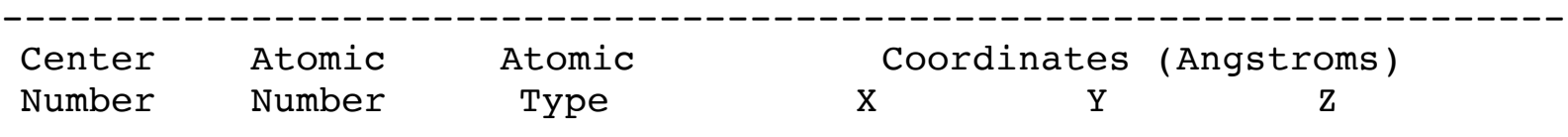

\begin{tabular}{|c|c|c|c|c|c|}
\hline 1 & 6 & 0 & 3.451798 & -2.202252 & 0.079066 \\
\hline 2 & 6 & 0 & 2.180819 & -1.644589 & 0.207960 \\
\hline 3 & 6 & 0 & 1.982919 & -0.259415 & 0.054450 \\
\hline 4 & 6 & 0 & 3.097457 & 0.551149 & -0.225223 \\
\hline 5 & 6 & 0 & 4.370710 & -0.005848 & -0.361081 \\
\hline 6 & 6 & 0 & 4.551719 & -1.384012 & -0.209005 \\
\hline
\end{tabular}

S-17 


\begin{tabular}{|c|c|c|c|c|c|}
\hline 7 & 1 & 0 & 3.590590 & -3.273530 & 0.206540 \\
\hline 8 & 1 & 0 & 1.325569 & -2.275080 & 0.440930 \\
\hline 9 & 1 & 0 & 2.948785 & 1.622463 & -0.334133 \\
\hline 10 & 1 & 0 & 5.222249 & 0.634198 & -0.579870 \\
\hline 11 & 1 & 0 & 5.543399 & -1.818840 & -0.308688 \\
\hline 12 & 6 & 0 & 0.656324 & 0.344079 & 0.188041 \\
\hline 13 & 6 & 0 & -0.603518 & -0.129075 & 0.303874 \\
\hline 14 & 5 & 0 & -0.356882 & 1.496843 & 0.261064 \\
\hline 15 & 8 & 0 & -2.551553 & -1.068138 & -0.899694 \\
\hline 16 & 8 & 0 & -2.054626 & -2.229368 & 1.028094 \\
\hline 17 & 6 & 0 & -3.329056 & -2.716517 & 0.575127 \\
\hline 18 & 1 & 0 & -3.331703 & -3.808996 & 0.620508 \\
\hline 19 & 1 & 0 & -4.108919 & -2.329913 & 1.241939 \\
\hline 20 & 6 & 0 & -3.473703 & -2.175365 & -0.862554 \\
\hline 21 & 1 & 0 & -3.168805 & -2.908425 & -1.618139 \\
\hline 22 & 1 & 0 & -4.481451 & -1.818181 & -1.091870 \\
\hline 23 & 8 & 0 & -0.864054 & 2.201914 & -1.004257 \\
\hline 24 & 8 & 0 & -0.613299 & 2.426771 & 1.329837 \\
\hline 25 & 6 & 0 & -0.715112 & 3.719794 & 0.769330 \\
\hline 26 & 1 & 0 & -1.334571 & 4.358359 & 1.410345 \\
\hline 27 & 1 & 0 & 0.281976 & 4.183605 & 0.676742 \\
\hline 28 & 6 & 0 & -1.332550 & 3.498984 & -0.622947 \\
\hline 29 & 1 & 0 & -1.011583 & 4.241479 & -1.363500 \\
\hline 30 & 1 & 0 & -2.433107 & 3.505793 & -0.572412 \\
\hline 31 & 5 & 0 & -1.650249 & -1.201716 & 0.211327 \\
\hline 32 & 3 & 0 & -1.788452 & 0.660132 & -1.368142 \\
\hline
\end{tabular}

\section{IM3-S1}

Standard orientation:

\begin{tabular}{|c|c|c|c|c|c|}
\hline \multirow{2}{*}{$\begin{array}{l}\text { Center } \\
\text { Number }\end{array}$} & Atomic & Atomic & \multicolumn{3}{|c|}{ Coordinates (Angstroms) } \\
\hline & Number & Type & $\mathrm{X}$ & $\mathrm{Y}$ & $\mathrm{Z}$ \\
\hline 1 & 6 & 0 & 4.277944 & -0.257468 & 1.222325 \\
\hline 2 & 6 & 0 & 2.901362 & -0.247451 & 1.234448 \\
\hline 3 & 6 & 0 & 2.167596 & -0.236433 & 0.001572 \\
\hline 4 & 6 & 0 & 2.914700 & -0.243943 & -1.223326 \\
\hline 5 & 6 & 0 & 4.290992 & -0.253822 & -1.196270 \\
\hline 6 & 6 & 0 & 4.991912 & -0.261469 & 0.016891 \\
\hline 7 & 1 & 0 & 4.815358 & -0.262182 & 2.165797 \\
\hline 8 & 1 & 0 & 2.354624 & -0.246599 & 2.170279 \\
\hline 9 & 1 & 0 & 2.378002 & -0.240100 & -2.164924 \\
\hline 10 & 1 & 0 & 4.838627 & -0.255551 & -2.133855 \\
\hline 11 & 1 & 0 & 6.075895 & -0.271571 & 0.022715 \\
\hline 12 & 6 & 0 & 0.810725 & -0.247327 & -0.006569 \\
\hline 13 & 6 & 0 & -0.486104 & -0.063888 & -0.015038 \\
\hline 14 & 5 & 0 & -1.426838 & -1.295292 & -0.016937 \\
\hline 15 & 8 & 0 & -2.391697 & 1.677289 & 0.058270 \\
\hline 16 & 8 & 0 & -0.274638 & 2.518892 & -0.059322 \\
\hline 17 & 6 & 0 & -1.143653 & 3.653632 & -0.155028 \\
\hline 18 & 1 & 0 & -0.803593 & 4.421751 & 0.542133 \\
\hline 19 & 1 & 0 & -1.086036 & 4.046562 & -1.174186 \\
\hline 20 & 6 & 0 & -2.538485 & 3.111513 & 0.187470 \\
\hline & & & S-18 & & \\
\hline
\end{tabular}




$\begin{array}{rrrrrr}21 & 1 & 0 & -2.839764 & 3.326992 & 1.215070 \\ 22 & 1 & 0 & -3.322508 & 3.445500 & -0.492249 \\ 23 & 8 & 0 & -2.807946 & -1.177521 & -0.014372 \\ 24 & 8 & 0 & -1.021593 & -2.593181 & -0.019490 \\ 25 & 6 & 0 & -2.174264 & -3.433865 & 0.105183 \\ 26 & 1 & 0 & -2.107006 & -4.241727 & -0.625968 \\ 27 & 1 & 0 & -2.181146 & -3.863489 & 1.111061 \\ 28 & 6 & 0 & -3.369721 & -2.500811 & -0.143665 \\ 29 & 1 & 0 & -4.175780 & -2.609689 & 0.583363 \\ 30 & 1 & 0 & -3.782218 & -2.593751 & -1.151344 \\ 31 & 5 & 0 & -1.033213 & 1.393143 & -0.007569 \\ 32 & 3 & 0 & -3.918410 & 0.446256 & 0.055330\end{array}$

\section{Model Calculation of 2nd-borylation}

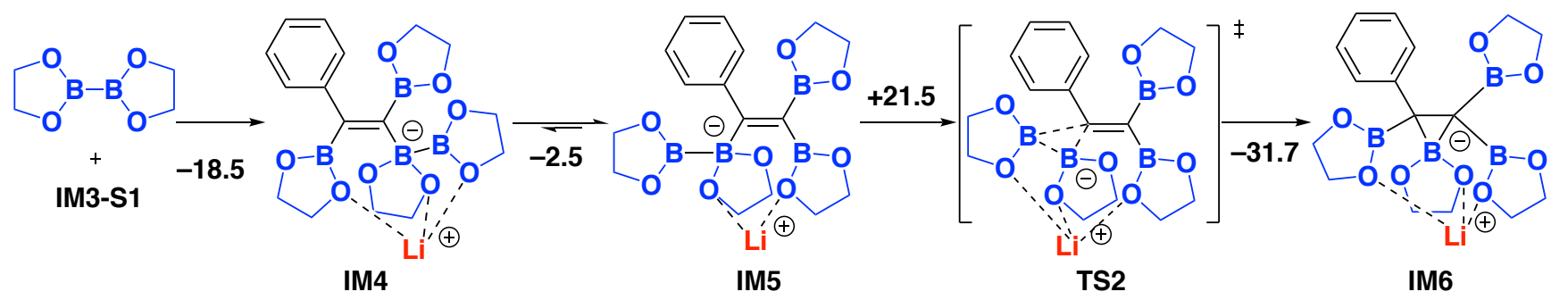

IM3-S1 and $\mathrm{B}_{2}(\mathrm{pin})_{2}$ forms a regioisomeric equilibrium between IM4 and IM5 and gives IM5 thermodynamically $\left(-2.5 \mathrm{kcal} \mathrm{mol}^{-1}\right)$. The second borylation from IM5 smoothly proceeds with lowered activation energy (+21.5 kcal mol $\left.{ }^{-1}\right)$ derived from an assist of lithium cation on TS2 and gives a large energy gain to IM6 $\left(-31.7 \mathrm{kcal} \mathrm{mol}^{-1}\right)$.

IM4

Sum of electronic and thermal Free Energies= $\quad-1331.184277$ Standard orientation:

\begin{tabular}{|c|c|c|c|c|c|}
\hline \multirow{2}{*}{$\begin{array}{l}\text { Center } \\
\text { Number }\end{array}$} & \multirow{2}{*}{$\begin{array}{l}\text { Atomic } \\
\text { Number }\end{array}$} & \multirow{2}{*}{$\begin{array}{l}\text { Atomic } \\
\text { Type }\end{array}$} & \multicolumn{3}{|c|}{ Coordinates (Angstroms) } \\
\hline & & & $\mathrm{X}$ & $\mathrm{Y}$ & Z \\
\hline 1 & 6 & 0 & 0.993510 & 0.384627 & 0.128493 \\
\hline 2 & 8 & 0 & 0.312208 & 2.650189 & -1.047330 \\
\hline 3 & 8 & 0 & 1.496594 & 2.815298 & 0.917762 \\
\hline 4 & 6 & 0 & 1.480096 & 4.135769 & 0.362900 \\
\hline 5 & 1 & 0 & 1.201484 & 4.853464 & 1.140063 \\
\hline 6 & 1 & 0 & 2.486664 & 4.376354 & 0.001010 \\
\hline 7 & 6 & 0 & 0.450798 & 4.066360 & -0.782303 \\
\hline 8 & 1 & 0 & -0.524452 & 4.469864 & -0.479899 \\
\hline 9 & 1 & 0 & 0.778479 & 4.566175 & -1.697780 \\
\hline 10 & 5 & 0 & 0.856033 & 1.946701 & 0.069645 \\
\hline 11 & 6 & 0 & -0.015134 & -0.458572 & 0.479880 \\
\hline 12 & 8 & 0 & -1.943680 & -0.218851 & 2.174527 \\
\hline 13 & 8 & 0 & -1.461836 & 1.681677 & 0.818233 \\
\hline 14 & 6 & 0 & -2.657674 & 0.889903 & 2.662062 \\
\hline 15 & 1 & 0 & -3.700121 & 0.894022 & 2.288995 \\
\hline 16 & 1 & 0 & -2.682739 & 0.866327 & 3.758455 \\
\hline 17 & 6 & 0 & -1.899120 & 2.107837 & 2.111499 \\
\hline & & & S-19 & & \\
\hline
\end{tabular}




\begin{tabular}{|c|c|c|c|c|c|}
\hline 18 & 1 & 0 & -1.024432 & 2.342338 & 2.733607 \\
\hline 19 & 1 & 0 & -2.526268 & 3.004746 & 2.018432 \\
\hline 20 & 6 & 0 & -3.410865 & -0.089615 & -2.682322 \\
\hline 21 & 6 & 0 & -4.138437 & -1.233225 & -1.946763 \\
\hline 22 & 1 & 0 & -4.090500 & 0.646285 & -3.121350 \\
\hline 23 & 1 & 0 & -2.723104 & -0.453804 & -3.452843 \\
\hline 24 & 1 & 0 & -5.192138 & -1.001007 & -1.753382 \\
\hline 25 & 1 & 0 & -4.077465 & -2.185094 & -2.481786 \\
\hline 26 & 5 & 0 & -2.601674 & -0.309704 & -0.491720 \\
\hline 27 & 8 & 0 & -3.457930 & -1.361184 & -0.685418 \\
\hline 28 & 8 & 0 & -2.633916 & 0.543552 & -1.638822 \\
\hline 29 & 5 & 0 & -1.506727 & 0.102351 & 0.815875 \\
\hline 30 & 3 & 0 & -1.462923 & 1.883299 & -1.017748 \\
\hline 31 & 6 & 0 & 2.381137 & -0.084399 & -0.203916 \\
\hline 32 & 6 & 0 & 3.002872 & 0.318795 & -1.401121 \\
\hline 33 & 6 & 0 & 3.113476 & -0.905332 & 0.673161 \\
\hline 34 & 6 & 0 & 4.295055 & -0.101726 & -1.724452 \\
\hline 35 & 1 & 0 & 2.456550 & 0.951953 & -2.097896 \\
\hline 36 & 6 & 0 & 4.407592 & -1.324002 & 0.352792 \\
\hline 37 & 1 & 0 & 2.663568 & -1.206409 & 1.614207 \\
\hline 38 & 6 & 0 & 5.004716 & -0.927202 & -0.847408 \\
\hline 39 & 1 & 0 & 4.746316 & 0.213310 & -2.662871 \\
\hline 40 & 1 & 0 & 4.954594 & -1.955262 & 1.049753 \\
\hline 41 & 1 & 0 & 6.012573 & -1.252522 & -1.093829 \\
\hline 42 & 5 & 0 & 0.127888 & -2.012781 & 0.486802 \\
\hline 43 & 8 & 0 & -0.255872 & -2.786416 & -0.594017 \\
\hline 44 & 8 & 0 & 0.540581 & -2.775749 & 1.558052 \\
\hline 45 & 6 & 0 & -0.219843 & -4.166854 & -0.192952 \\
\hline 46 & 1 & 0 & -1.250566 & -4.529745 & -0.102639 \\
\hline 47 & 1 & 0 & 0.297352 & -4.749643 & -0.961801 \\
\hline 48 & 6 & 0 & 0.522665 & -4.157438 & 1.163259 \\
\hline 49 & 1 & 0 & 1.557642 & -4.509756 & 1.075433 \\
\hline 50 & 1 & 0 & 0.013470 & -4.742016 & 1.935585 \\
\hline
\end{tabular}

\section{IM5}

Sum of electronic and thermal Free Energies= $\quad-1331.188294$ Standard orientation:

\begin{tabular}{|c|c|c|c|c|c|}
\hline Center & Atomic & Atomic & \multicolumn{3}{|c|}{ Coordinates (Angstroms) } \\
\hline Number & Number & Type & $\mathrm{x}$ & $\mathrm{Y}$ & $\mathrm{Z}$ \\
\hline 1 & 6 & 0 & -0.727031 & 3.878667 & -0.941482 \\
\hline 2 & 6 & 0 & -0.621505 & 2.489566 & -0.838219 \\
\hline 3 & 6 & 0 & -0.126699 & 1.879057 & 0.330286 \\
\hline 4 & 6 & 0 & 0.288161 & 2.712752 & 1.385793 \\
\hline 5 & 6 & 0 & 0.163734 & 4.099737 & 1.290687 \\
\hline 6 & 6 & 0 & -0.343327 & 4.690916 & 0.128844 \\
\hline 7 & 1 & 0 & -1.104937 & 4.325342 & -1.858984 \\
\hline 8 & 1 & 0 & -0.907786 & 1.864873 & -1.682097 \\
\hline 9 & 1 & 0 & 0.703599 & 2.259179 & 2.279281 \\
\hline 10 & 1 & 0 & 0.473638 & 4.723624 & 2.126154 \\
\hline 11 & 1 & 0 & -0.427276 & 5.772734 & 0.054605 \\
\hline 12 & 6 & 0 & $\begin{array}{c}-1.055198 \\
S-20\end{array}$ & -0.407463 & 0.079458 \\
\hline
\end{tabular}




\begin{tabular}{|c|c|c|c|c|c|}
\hline 13 & 5 & 0 & -2.500881 & 0.136367 & -0.157661 \\
\hline 14 & 8 & 0 & -0.325932 & -2.710333 & -1.011012 \\
\hline 15 & 8 & 0 & -1.885986 & -2.778905 & 0.670962 \\
\hline 16 & 6 & 0 & -1.891655 & -4.084679 & 0.084844 \\
\hline 17 & 1 & 0 & -1.858575 & -4.839388 & 0.876015 \\
\hline 18 & 1 & 0 & -2.817515 & -4.209001 & -0.489305 \\
\hline 19 & 6 & 0 & -0.644048 & -4.107637 & -0.817544 \\
\hline 20 & 1 & 0 & 0.205395 & -4.602044 & -0.327719 \\
\hline 21 & 1 & 0 & -0.817428 & -4.569696 & -1.793173 \\
\hline 22 & 8 & 0 & -3.304939 & -0.330460 & -1.190300 \\
\hline 23 & 8 & 0 & -3.167024 & 1.031419 & 0.650801 \\
\hline 24 & 6 & 0 & -4.437723 & 1.319596 & 0.050311 \\
\hline 25 & 1 & 0 & -5.210356 & 1.317628 & 0.825168 \\
\hline 26 & 1 & 0 & -4.392407 & 2.317559 & -0.403580 \\
\hline 27 & 6 & 0 & -4.626467 & 0.204453 & -1.001015 \\
\hline 28 & 1 & 0 & -5.007283 & 0.572410 & -1.958841 \\
\hline 29 & 1 & 0 & -5.282228 & -0.599401 & -0.643506 \\
\hline 30 & 5 & 0 & -1.027817 & -1.957192 & -0.025327 \\
\hline 31 & 6 & 0 & 0.004060 & 0.393801 & 0.417392 \\
\hline 32 & 8 & 0 & 1.892006 & 0.240518 & 2.178265 \\
\hline 33 & 8 & 0 & 1.417063 & -1.744819 & 0.959583 \\
\hline 34 & 6 & 0 & 2.542607 & -0.847291 & 2.789905 \\
\hline 35 & 1 & 0 & 3.599679 & -0.913671 & 2.468781 \\
\hline 36 & 1 & 0 & 2.518286 & -0.730997 & 3.880432 \\
\hline 37 & 6 & 0 & 1.766292 & -2.077926 & 2.302909 \\
\hline 38 & 1 & 0 & 0.853315 & -2.226707 & 2.896979 \\
\hline 39 & 1 & 0 & 2.359016 & -3.002311 & 2.318421 \\
\hline 40 & 6 & 0 & 3.499328 & -0.313799 & -2.603574 \\
\hline 41 & 6 & 0 & 4.155117 & 0.934323 & -1.978997 \\
\hline 42 & 1 & 0 & 4.221550 & -1.068888 & -2.926499 \\
\hline 43 & 1 & 0 & 2.834066 & -0.069295 & -3.438774 \\
\hline 44 & 1 & 0 & 5.205196 & 0.764856 & -1.714409 \\
\hline 45 & 1 & 0 & 4.090158 & 1.815331 & -2.623545 \\
\hline 46 & 5 & 0 & 2.598608 & 0.120637 & -0.481083 \\
\hline 47 & 8 & 0 & 3.412190 & 1.182633 & -0.771837 \\
\hline 48 & 8 & 0 & 2.700229 & -0.852197 & -1.523698 \\
\hline 49 & 5 & 0 & 1.476201 & -0.184150 & 0.833098 \\
\hline 50 & 3 & 0 & 1.485536 & -2.122578 & -0.830083 \\
\hline
\end{tabular}

TS2

Sum of electronic and thermal Free Energies= $\quad-1331.153991$ Standard orientation:

\begin{tabular}{cccccc} 
Center & Atomic & Atomic & \multicolumn{2}{c}{ Coordinates } & (Angstroms) \\
Number & Number & Type & X & $Y$ & Z \\
-1 & 6 & 0 & -3.256782 & 0.711490 & -2.618271 \\
2 & 6 & 0 & -2.019998 & 0.667746 & -1.971311 \\
3 & 6 & 0 & -1.677730 & -0.394062 & -1.112688 \\
4 & 6 & 0 & -2.626752 & -1.422683 & -0.943082 \\
5 & 6 & 0 & -3.863485 & -1.377302 & -1.583758 \\
6 & 6 & 0 & -4.190808 & -0.307867 & -2.424389 \\
7 & 1 & 0 & -3.484155 & 1.542881 & -3.282031
\end{tabular}




\begin{tabular}{|c|c|c|c|c|c|}
\hline 8 & 1 & 0 & -1.289102 & 1.447438 & -2.157778 \\
\hline 9 & 1 & 0 & -2.398742 & -2.264763 & -0.300261 \\
\hline 10 & 1 & 0 & -4.575346 & -2.184648 & -1.426753 \\
\hline 11 & 1 & 0 & -5.155201 & -0.276241 & -2.926024 \\
\hline 12 & 6 & 0 & 0.544753 & 0.735892 & -0.446558 \\
\hline 13 & 5 & 0 & 0.042985 & 2.073112 & 0.060599 \\
\hline 14 & 8 & 0 & 3.056171 & 0.948299 & -0.001510 \\
\hline 15 & 8 & 0 & 2.520291 & 0.273101 & -2.131223 \\
\hline 16 & 6 & 0 & 3.945863 & 0.169231 & -2.029265 \\
\hline 17 & 1 & 0 & 4.220769 & -0.892483 & -1.993629 \\
\hline 18 & 1 & 0 & 4.405903 & 0.623039 & -2.912215 \\
\hline 19 & 6 & 0 & 4.302373 & 0.906951 & -0.720959 \\
\hline 20 & 1 & 0 & 5.056394 & 0.387910 & -0.121250 \\
\hline 21 & 1 & 0 & 4.632011 & 1.937223 & -0.897777 \\
\hline 22 & 8 & 0 & 0.703944 & 3.292000 & -0.148433 \\
\hline 23 & 8 & 0 & -1.103284 & 2.268804 & 0.847488 \\
\hline 24 & 6 & 0 & -1.325095 & 3.679081 & 0.957864 \\
\hline 25 & 1 & 0 & -2.099382 & 3.978084 & 0.238157 \\
\hline 26 & 1 & 0 & -1.675116 & 3.918698 & 1.967969 \\
\hline 27 & 6 & 0 & 0.044501 & 4.299762 & 0.623183 \\
\hline 28 & 1 & 0 & 0.634205 & 4.507728 & 1.527963 \\
\hline 29 & 1 & 0 & -0.030062 & 5.220849 & 0.035639 \\
\hline 30 & 5 & 0 & 1.998512 & 0.665400 & -0.916523 \\
\hline 31 & 6 & 0 & -0.291981 & -0.450724 & -0.543405 \\
\hline 32 & 8 & 0 & -0.065019 & -3.114305 & -0.462154 \\
\hline 33 & 8 & 0 & 1.707532 & -1.821904 & 0.346242 \\
\hline 34 & 6 & 0 & 0.861980 & -3.998557 & 0.155893 \\
\hline 35 & 1 & 0 & 0.491481 & -4.300944 & 1.148344 \\
\hline 36 & 1 & 0 & 0.978581 & -4.894733 & -0.462369 \\
\hline 37 & 6 & 0 & 2.159046 & -3.178430 & 0.276032 \\
\hline 38 & 1 & 0 & 2.794802 & -3.295734 & -0.612053 \\
\hline 39 & 1 & 0 & 2.743762 & -3.426029 & 1.169762 \\
\hline 40 & 6 & 0 & -0.407807 & 0.036977 & 3.510726 \\
\hline 41 & 6 & 0 & -1.795458 & -0.614865 & 3.351870 \\
\hline 42 & 1 & 0 & 0.099386 & -0.235205 & 4.440336 \\
\hline 43 & 1 & 0 & -0.450356 & 1.126015 & 3.410436 \\
\hline 44 & 1 & 0 & -1.933002 & -1.480606 & 4.009531 \\
\hline 45 & 1 & 0 & -2.616592 & 0.089832 & 3.504999 \\
\hline 46 & 5 & 0 & -0.579446 & -0.988979 & 1.450016 \\
\hline 47 & 8 & 0 & -1.827962 & -1.071909 & 1.983815 \\
\hline 48 & 8 & 0 & 0.346689 & -0.494014 & 2.393235 \\
\hline 49 & 5 & 0 & 0.304870 & -1.794141 & -0.115286 \\
\hline 50 & 3 & 0 & 1.897516 & -0.107231 & 1.244666 \\
\hline
\end{tabular}

\section{IM6}

Sum of electronic and thermal Free Energies= $\quad-1331.204467$ Standard orientation:

\begin{tabular}{|c|c|c|c|c|c|}
\hline Center & Atomic & Atomic & \multicolumn{3}{|c|}{ Coordinates (Angstroms) } \\
\hline Number & Number & Type & $\mathrm{X}$ & $\mathrm{Y}$ & $\mathrm{Z}$ \\
\hline 1 & 6 & 0 & -3.922855 & -1.796585 & 0.027757 \\
\hline 2 & 6 & 0 & $\begin{array}{r}-2.623057 \\
S-22\end{array}$ & -1.502690 & 0.451195 \\
\hline
\end{tabular}




\begin{tabular}{|c|c|c|c|c|c|}
\hline 3 & 6 & 0 & -1.669187 & -0.987172 & -0.437808 \\
\hline 4 & 6 & 0 & -2.060785 & -0.775957 & -1.769843 \\
\hline 5 & 6 & 0 & -3.355925 & -1.064717 & -2.199839 \\
\hline 6 & 6 & 0 & -4.296539 & -1.578700 & -1.300447 \\
\hline 7 & 1 & 0 & -4.645192 & -2.191913 & 0.739248 \\
\hline 8 & 1 & 0 & -2.343989 & -1.663209 & 1.488792 \\
\hline 9 & 1 & 0 & -1.337792 & -0.366348 & -2.472581 \\
\hline 10 & 1 & 0 & -3.633116 & -0.888838 & -3.237413 \\
\hline 11 & 1 & 0 & -5.307874 & -1.804090 & -1.631670 \\
\hline 12 & 6 & 0 & 0.320308 & 0.763953 & -0.057188 \\
\hline 13 & 5 & 0 & -0.636679 & 1.973972 & -0.147978 \\
\hline 14 & 8 & 0 & 2.950390 & 0.793665 & -0.163576 \\
\hline 15 & 8 & 0 & 1.839181 & 1.741382 & -1.921297 \\
\hline 16 & 6 & 0 & 3.220931 & 2.057590 & -2.115562 \\
\hline 17 & 1 & 0 & 3.492929 & 1.877350 & -3.159626 \\
\hline 18 & 1 & 0 & 3.377920 & 3.117844 & -1.882817 \\
\hline 19 & 6 & 0 & 3.963276 & 1.134625 & -1.132887 \\
\hline 20 & 1 & 0 & 4.315789 & 0.213094 & -1.613058 \\
\hline 21 & 1 & 0 & 4.799433 & 1.620655 & -0.622403 \\
\hline 22 & 8 & 0 & -0.120365 & 3.261612 & 0.020812 \\
\hline 23 & 8 & 0 & -2.003700 & 1.996610 & -0.328026 \\
\hline 24 & 6 & 0 & -2.463349 & 3.332308 & -0.087932 \\
\hline 25 & 1 & 0 & -3.220431 & 3.592692 & -0.834538 \\
\hline 26 & 1 & 0 & -2.918203 & 3.373477 & 0.909971 \\
\hline 27 & 6 & 0 & -1.188853 & 4.195056 & -0.183078 \\
\hline 28 & 1 & 0 & -1.138604 & 4.977799 & 0.581111 \\
\hline 29 & 1 & 0 & -1.074289 & 4.658075 & -1.172634 \\
\hline 30 & 5 & 0 & 1.669664 & 1.095969 & -0.714596 \\
\hline 31 & 6 & 0 & -0.243074 & -0.742950 & -0.002598 \\
\hline 32 & 8 & 0 & 0.196974 & -3.192753 & -0.679886 \\
\hline 33 & 8 & 0 & 2.063951 & -2.008214 & -0.073984 \\
\hline 34 & 6 & 0 & 1.251831 & -4.148645 & -0.576447 \\
\hline 35 & 1 & 0 & 1.109660 & -4.745399 & 0.333952 \\
\hline 36 & 1 & 0 & 1.225411 & -4.815427 & -1.443526 \\
\hline 37 & 6 & 0 & 2.533146 & -3.299574 & -0.513119 \\
\hline 38 & 1 & 0 & 3.005172 & -3.182951 & -1.496289 \\
\hline 39 & 1 & 0 & 3.273300 & -3.680271 & 0.197823 \\
\hline 40 & 6 & 0 & 1.032661 & -0.001885 & 3.561716 \\
\hline 41 & 6 & 0 & -0.497606 & 0.077372 & 3.612398 \\
\hline 42 & 1 & 0 & 1.457277 & -0.746218 & 4.245978 \\
\hline 43 & 1 & 0 & 1.487639 & 0.978833 & 3.765607 \\
\hline 44 & 1 & 0 & -0.930251 & -0.916013 & 3.822802 \\
\hline 45 & 1 & 0 & -0.863419 & 0.783572 & 4.366924 \\
\hline 46 & 5 & 0 & 0.116788 & 0.053295 & 1.381769 \\
\hline 47 & 8 & 0 & -0.864075 & 0.522254 & 2.322025 \\
\hline 48 & 8 & 0 & 1.295002 & -0.388174 & 2.209825 \\
\hline 49 & 5 & 0 & 0.647642 & -1.948899 & -0.278608 \\
\hline 50 & 3 & 0 & 2.672382 & -0.615877 & 1.055455 \\
\hline
\end{tabular}




\section{Single Crystal Structure}

$\underline{\mathbf{3 a}}$

Crystal data: $\mathrm{C}_{33} \mathrm{H}_{56} \mathrm{~B}_{4} \mathrm{O}_{9}, F W=640.04$, monoclinic P21/n (\#14), $a=10.8513(2), b=18.0770(4), c=19.3841(4)$ $\AA, \beta=103.4340(7)^{\circ}, V=3698.3(2) \AA^{3} ; D_{\mathrm{X}}=1.149 \mathrm{Mg} \mathrm{m}^{-3} ; Z=4 ; \mu(\mathrm{Cu} K \alpha)=0.640 \mathrm{~mm}^{-1}, T=93 \mathrm{~K}$. Prism colorless crystals were grown from a hexane solution of 3a. A single crystal with the dimensions of $0.300 \mathrm{x}$ $0.250 \times 0.200 \mathrm{~mm}$ was mounted on a glass capillary and set on a Rigaku XtaLAB Synergy-S with Rigaku HyPix-6000 area detector. The diffraction data were collected using $\mathrm{Cu} K \alpha$ radiation, which was monochromated by a multi-layered confocal mirror. The unit cell dimensions were determined using 47094 reflections with 3.38 $\leq 2 \theta \leq 68.24^{\circ}$. The diffraction data of 47094 within $3.38 \leq 2 \theta \leq 68.24^{\circ}$ were collected and merged to give 6773 unique reflections with the Rint of 0.0667 . The structure was solved by a direct method and refined on $F 2$ by a least-squares method by the programs SIR20046 and SHELXL2018/1 respectively. The final $R$ values against 6773 unique reflections $\left(2 \theta \max =60.64^{\circ}\right)$ with $I>2 \sigma(I)$ are 0.0667 and 0.1454 for the $R(F)$ and the $w R(F 2)$, respectively. Crystallographic data have been deposited with the Cambridge Crystallographic Data Centre: Deposition code CCDC 1912516.

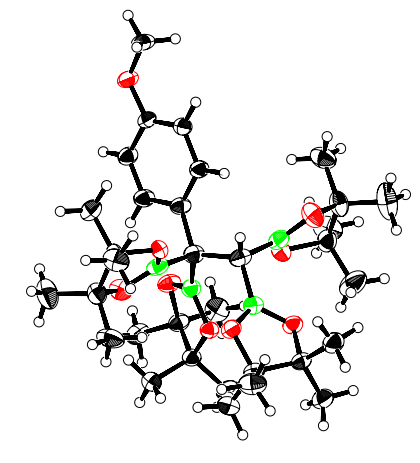

$\underline{4 a}$

Crystal data: $\mathrm{C}_{27} \mathrm{H}_{45} \mathrm{~B}_{3} \mathrm{O}_{7}, F W=514.08$, monoclinic P21/n (\#14), $a=11.6652(2), b=22.1573(4), c=11.8208(2)$ $\AA, \beta=104.2787(7)^{\circ}, V=2960.94(9) \AA^{3} ; D_{\mathrm{X}}=1.153 \mathrm{Mg} \mathrm{m}^{-3} ; Z=4 ; \mu(\mathrm{CuK \alpha})=0.639 \mathrm{~mm}^{-1}, T=93 \mathrm{~K}$. Prism colorless crystals were grown from a hexane solution of 4a. A single crystal with the dimensions of $0.300 \mathrm{x}$ $0.300 \times 0.300 \mathrm{~mm}$ was mounted on a glass capillary and set on a Rigaku XtaLAB Synergy-S with Rigaku HyPix-6000 area detector. The diffraction data were collected using $\mathrm{Cu} K \alpha$ radiation, which was monochromated by a multi-layered confocal mirror. The unit cell dimensions were determined using 13935 reflections with 4.35 $\leq 2 \theta \leq 68.22^{\circ}$. The diffraction data of 13935 within $3.38 \leq 2 \theta \leq 68.17^{\circ}$ were collected and merged to give 5392 unique reflections with the Rint of 0.0856 . The structure was solved by a direct method and refined on $F 2$ by a least-squares method by the programs SIR20046 and SHELXL2018/1 respectively. The final $R$ values against 5392 unique reflections $\left(2 \theta \max =68.22^{\circ}\right)$ with $I>2 \sigma(I)$ are 0.0856 and 0.1515 for the $R(F)$ and the $w R(F 2)$, respectively.

Crystallographic data have been deposited with the Cambridge Crystallographic Data Centre: Deposition code CCDC 1912517. 


\section{References}

(1) Gaussian 09, Revision B.01, Frisch, M. J.; Trucks, G. W.; Schlegel, H. B.; Scuseria, G. E.; Robb, M. A.; Cheeseman, J. R.; Scalmani, G.; Barone, V.; Mennucci, B.; Petersson, G. A.; Nakatsuji, H.; Caricato, M.; Li, X.; Hratchian, H. P.; Izmaylov, A. F.; Bloino, J.; Zheng, G.; Sonnenberg, J. L.; Hada, M.; Ehara, M.; Toyota, K.; Fukuda, R.; Hasegawa, J.; Ishida, M.; Nakajima, T.; Honda, Y.; Kitao, O.; Nakai, H.; Vreven, T.; Montgomery, Jr., J. A.; Peralta, J. E.; Ogliaro, F.; Bearpark, M.; Heyd, J. J.; Brothers, E.; Kudin, K. N.; Staroverov, V. N.; Kobayashi, R.; Normand, J.; Raghavachari, K.; Rendell, A.; Burant, J. C.; Iyengar, S. S.; Tomasi, J.; Cossi, M.; Rega, N.; Millam, N. J.; Klene, M.; Knox, J. E.; Cross, J. B.; Bakken, V.; Adamo, C.; Jaramillo, J.; Gomperts, R.; Stratmann, R. E.; Yazyev, O.; Austin, A. J.; Cammi, R.; Pomelli, C.; Ochterski, J. W.; Martin, R. L.; Morokuma, K.; Zakrzewski, V. G.; Voth, G. A.; Salvador, P.; Dannenberg, J. J.; Dapprich, S.; Daniels, A. D.; Farkas, Ö.; Foresman, J. B.; Ortiz, J. V.; Cioslowski, J.; Fox, D. J. Gaussian, Inc., Wallingford CT, 2009.

(2) (a) Beche, A. D. Phys. Rev. 1988, A38, 3098-3100. (b) Beche, A. D. J. Chem. Phys. 1993, 98. 1372-1377. (c) Beche, A. D. J. Chem. Phys. 1993, 98, 5648-5652. (d) Lee, C; Yang, W.; Parr, R. G. Phys. Rev. 1988, B37, 785-788.

(3) (a) Fukui, K. Acc. Chem. Res. 1981, 14, 363-368. (b) Ishida, K.; Morokuma, K.; Komornicki, A. J. Chem. Phys. 1977, 66, 2153-2156. (c) Gonzalez, C.; Schlegel, H. B. J. Chem. Phys. 1989, 90, 2154-2161. (d) Schlegel, H. B.; Gonzalez, C. J. Phys. Chem. 1990, 94, 5523-5527. 


\section{Copies of NMR Spectra}
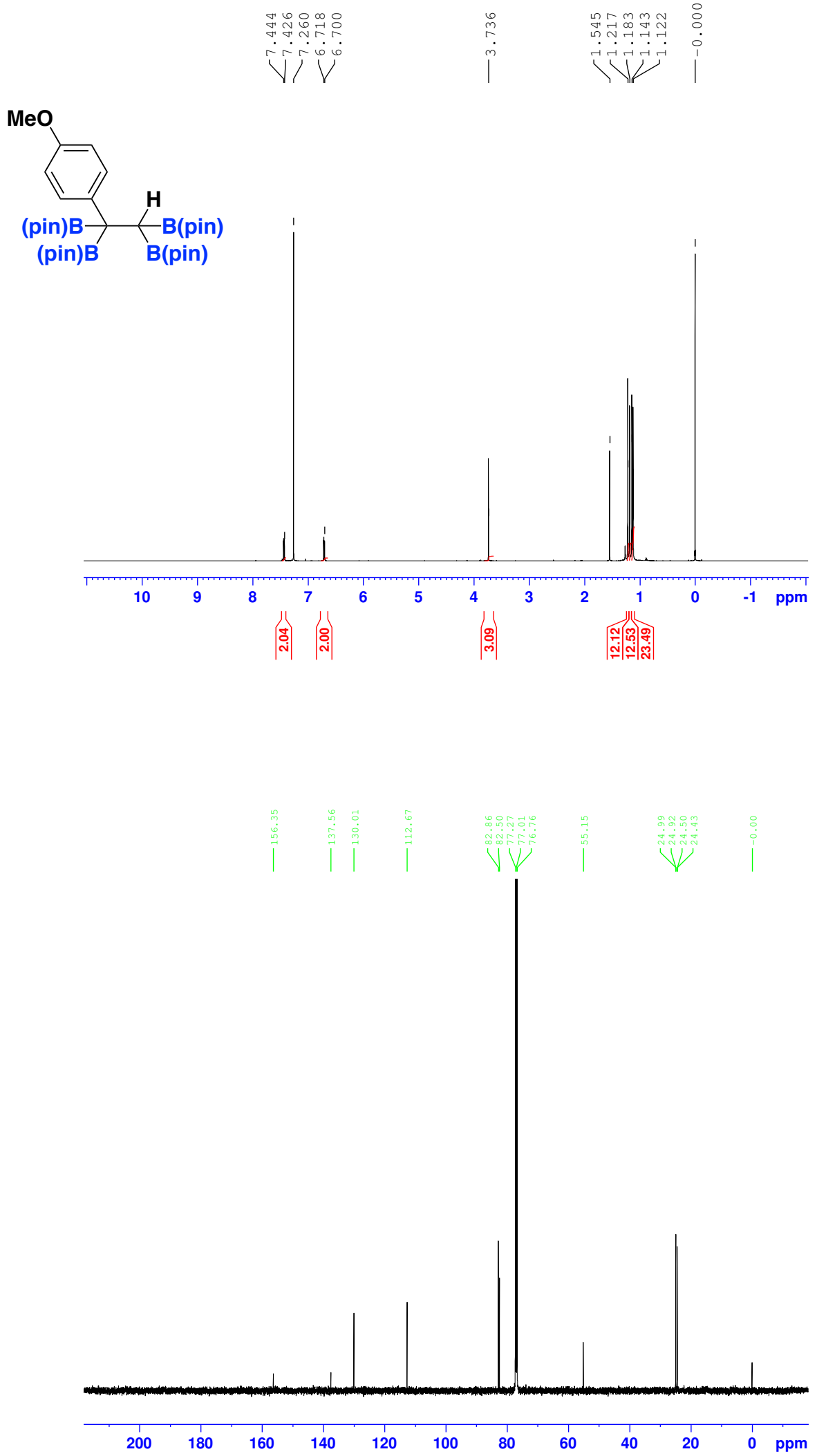

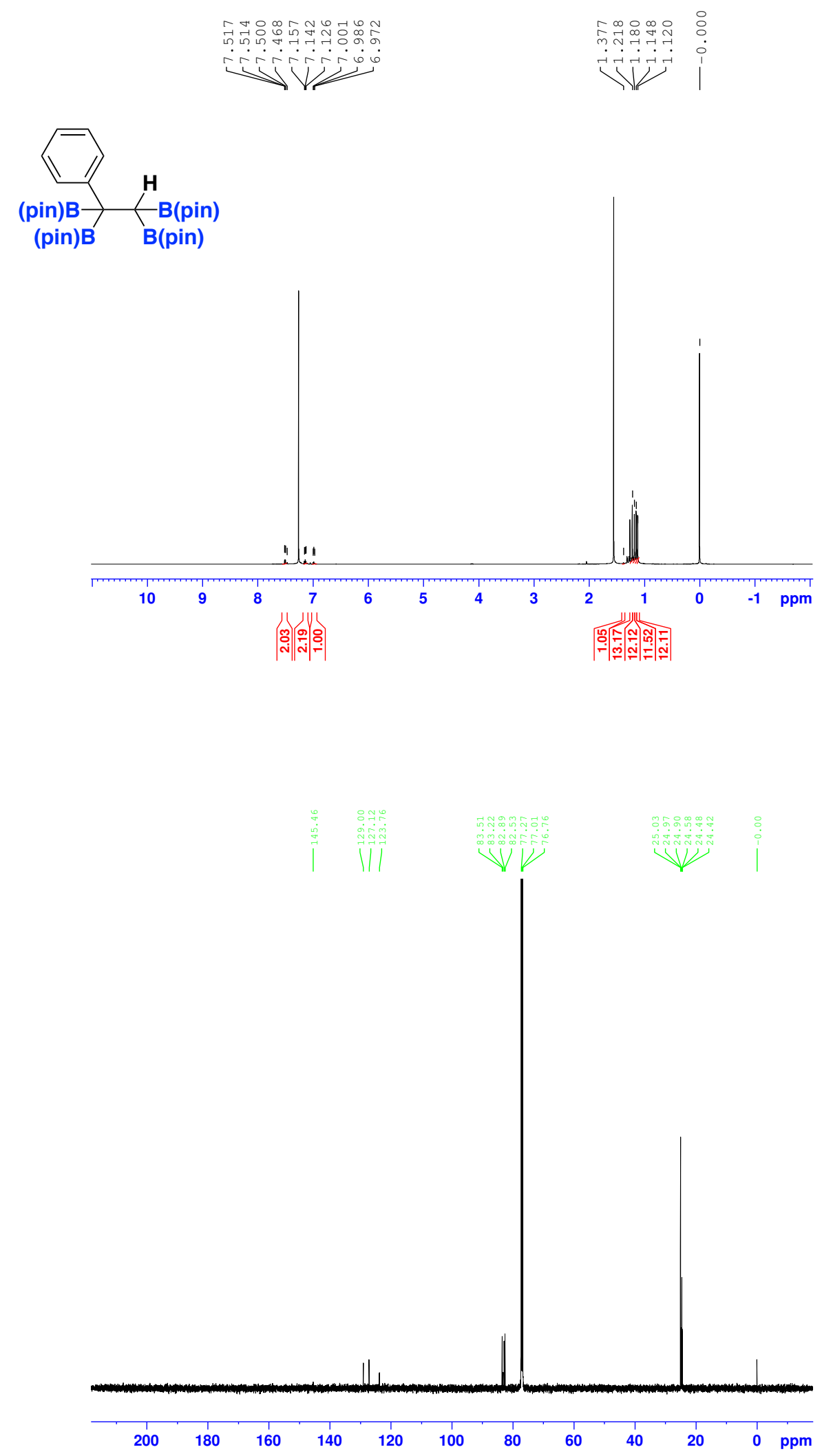

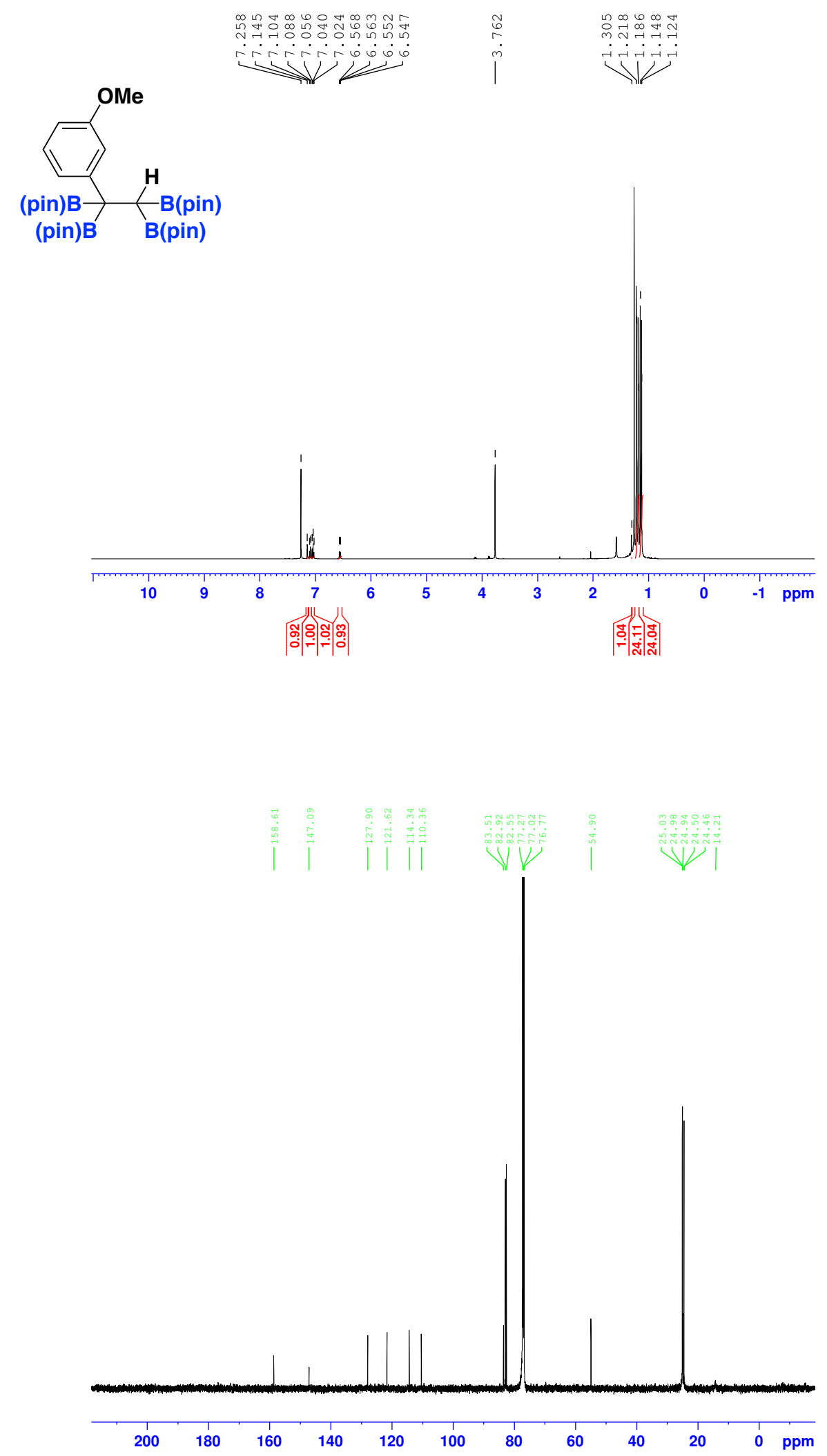

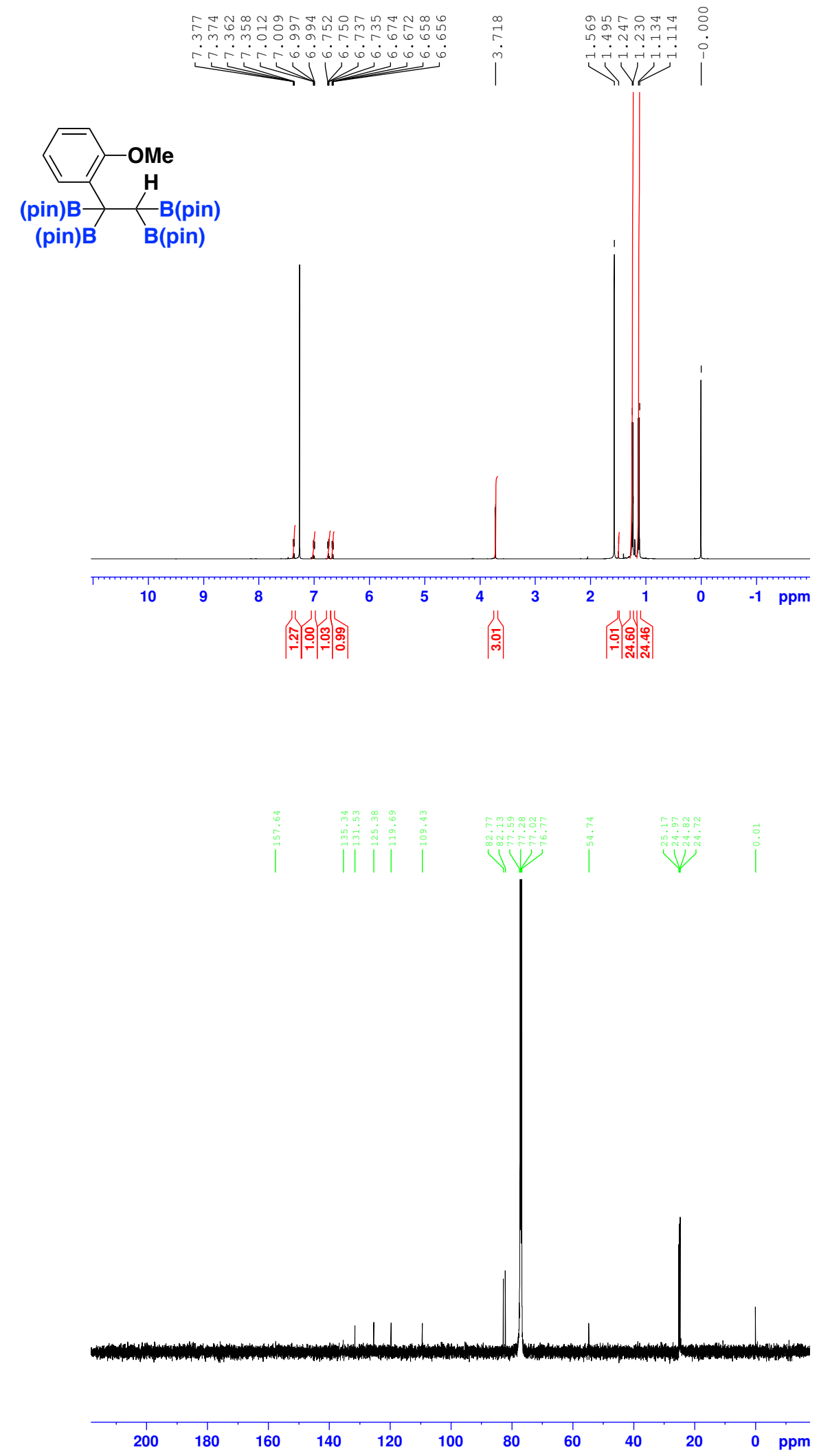

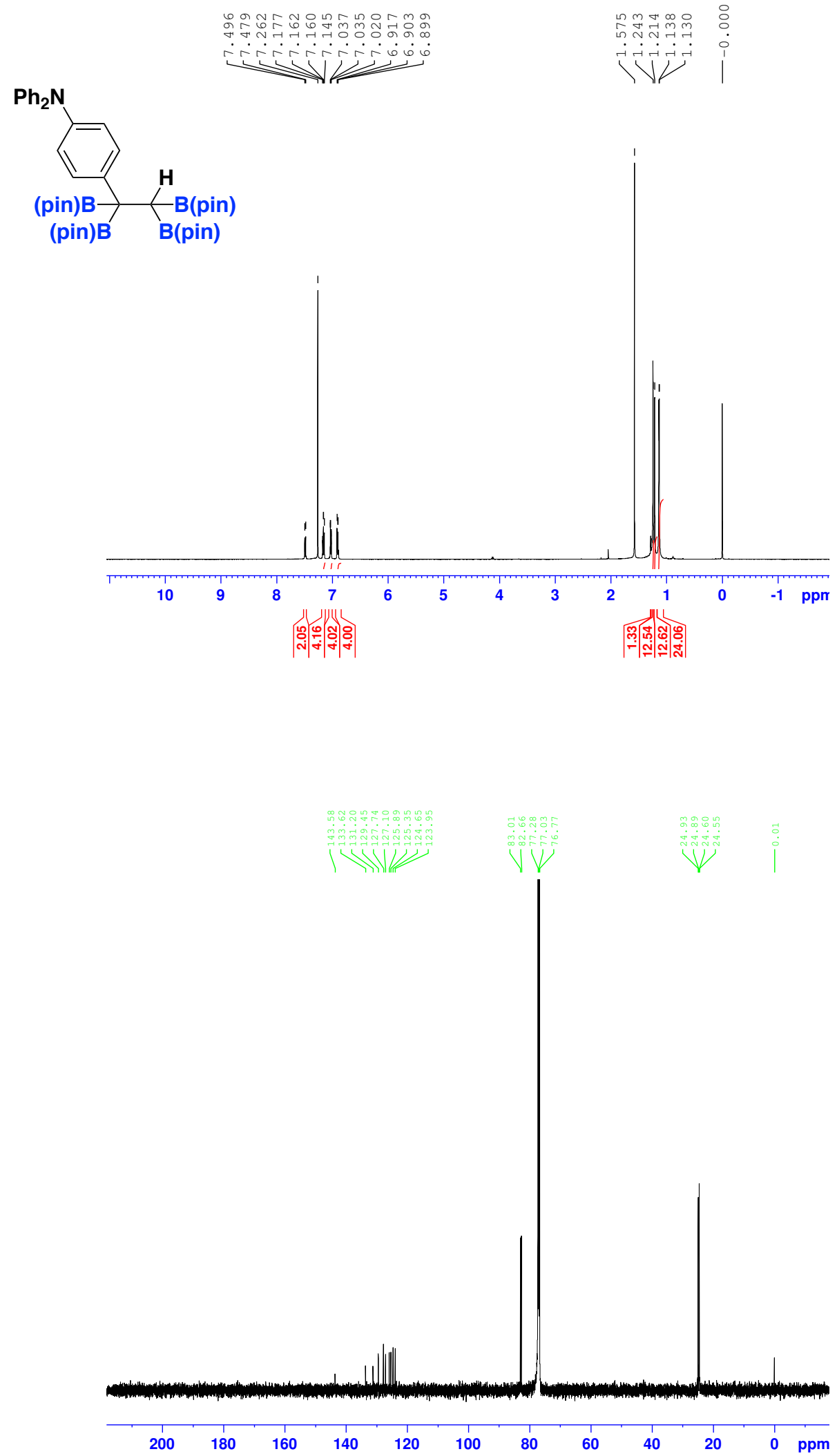

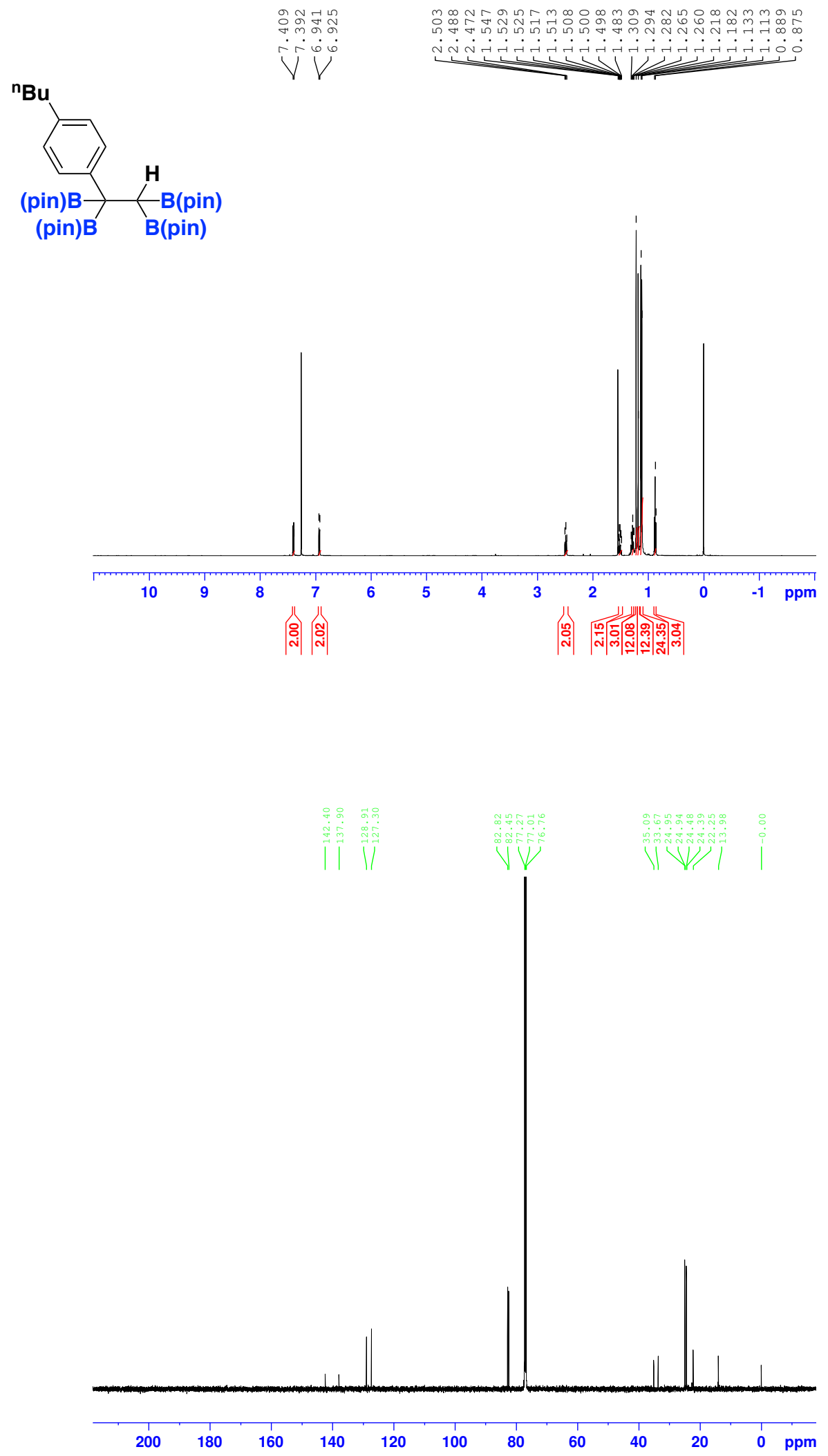


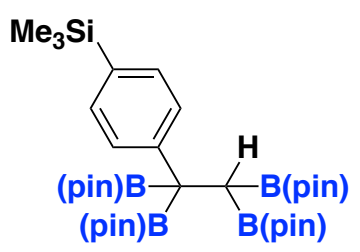

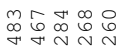

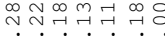

N

नंनંनं

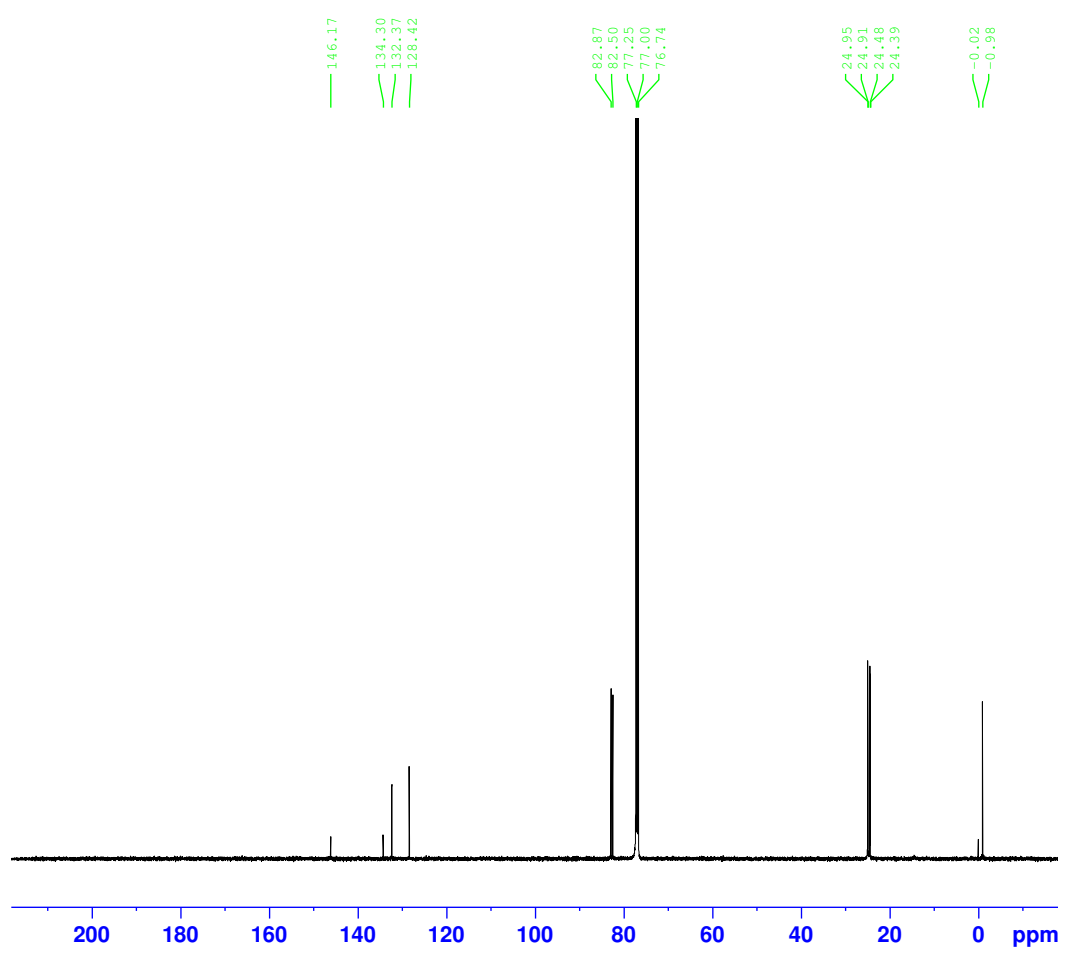



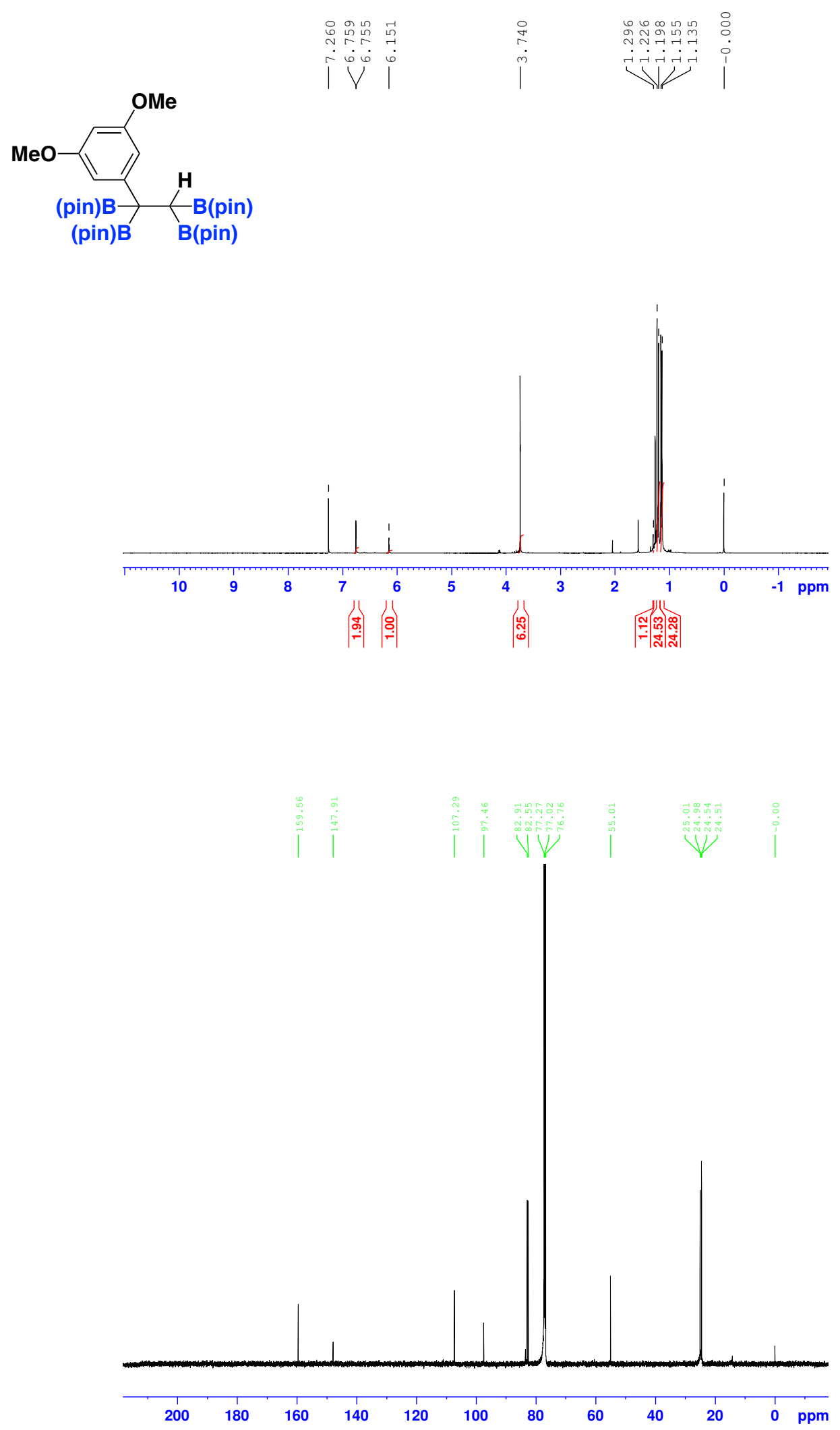

S-33 

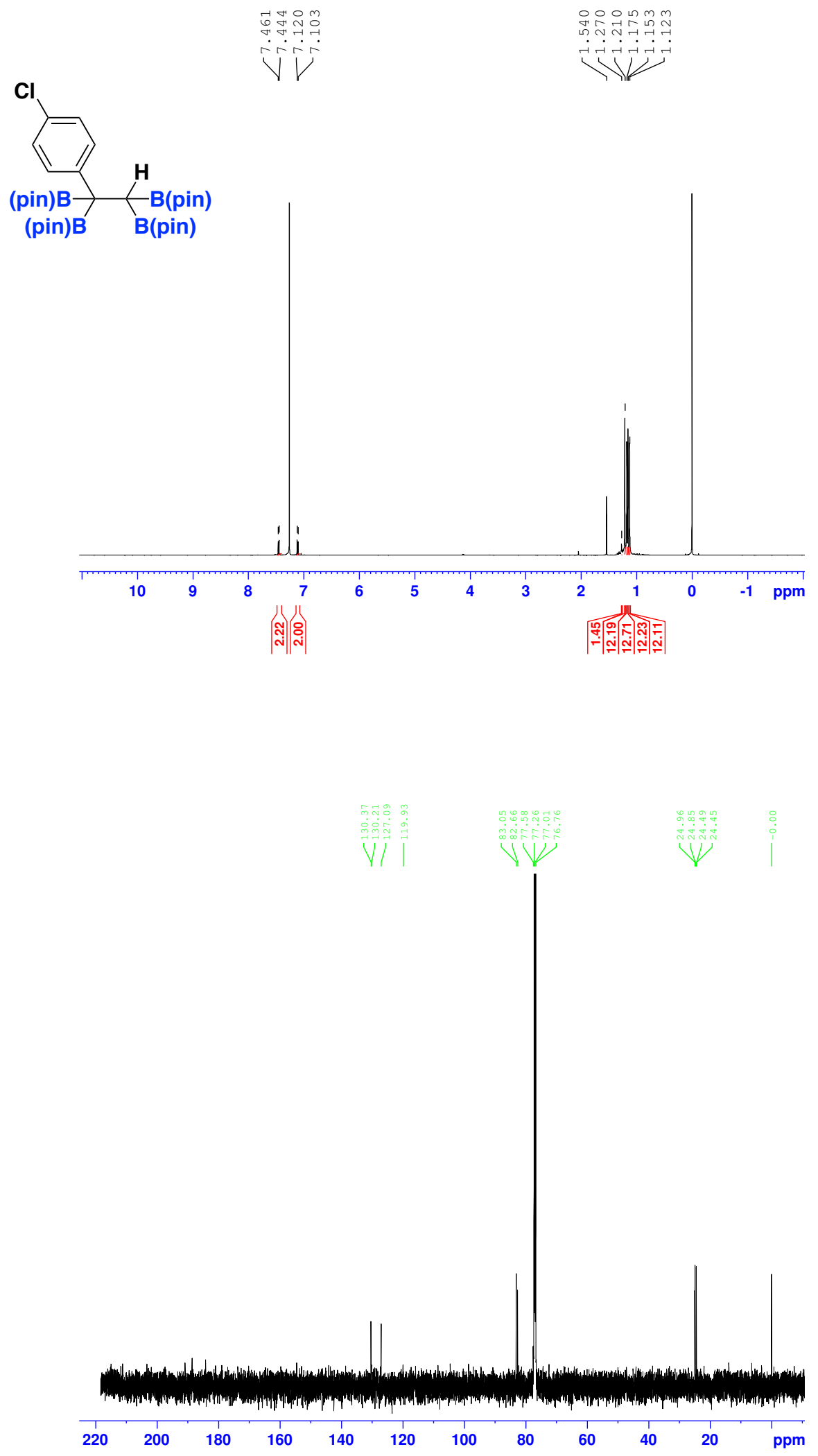

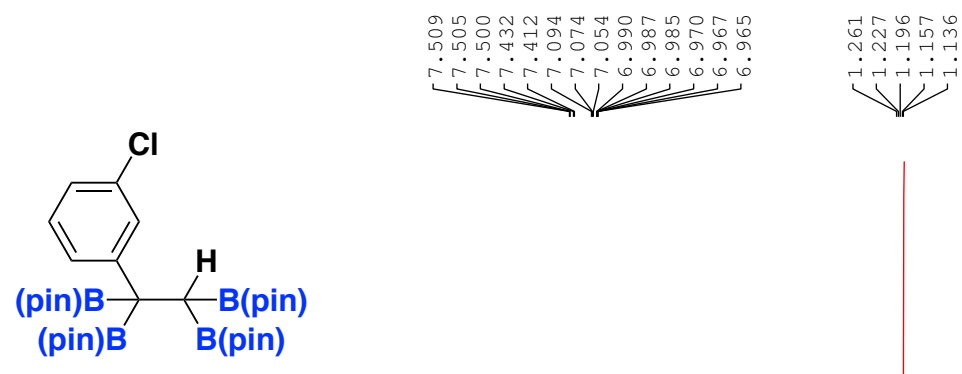

(pin) $B \quad B($ pin)
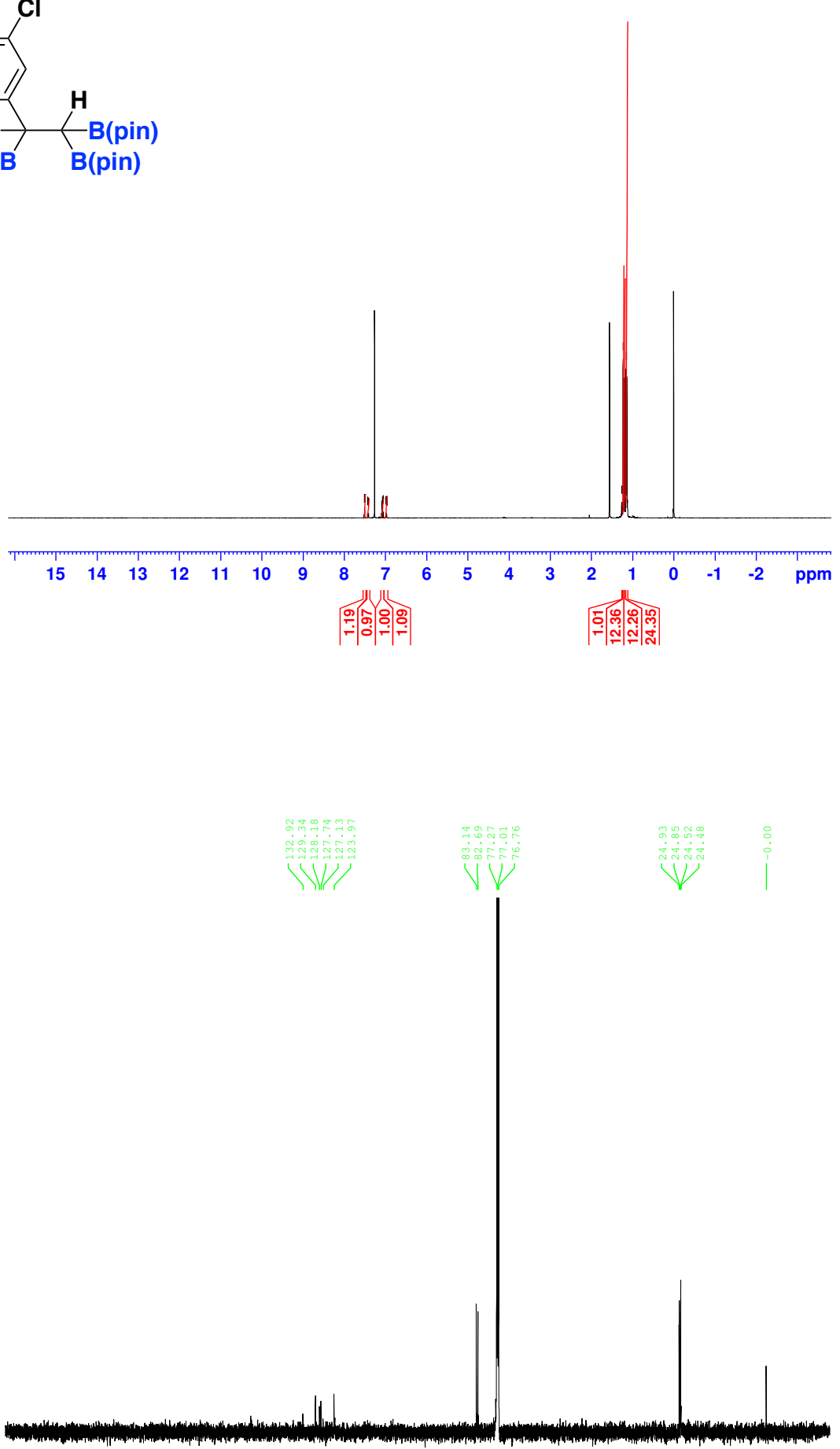

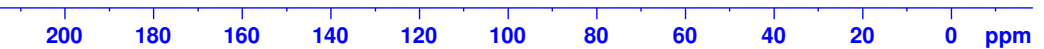



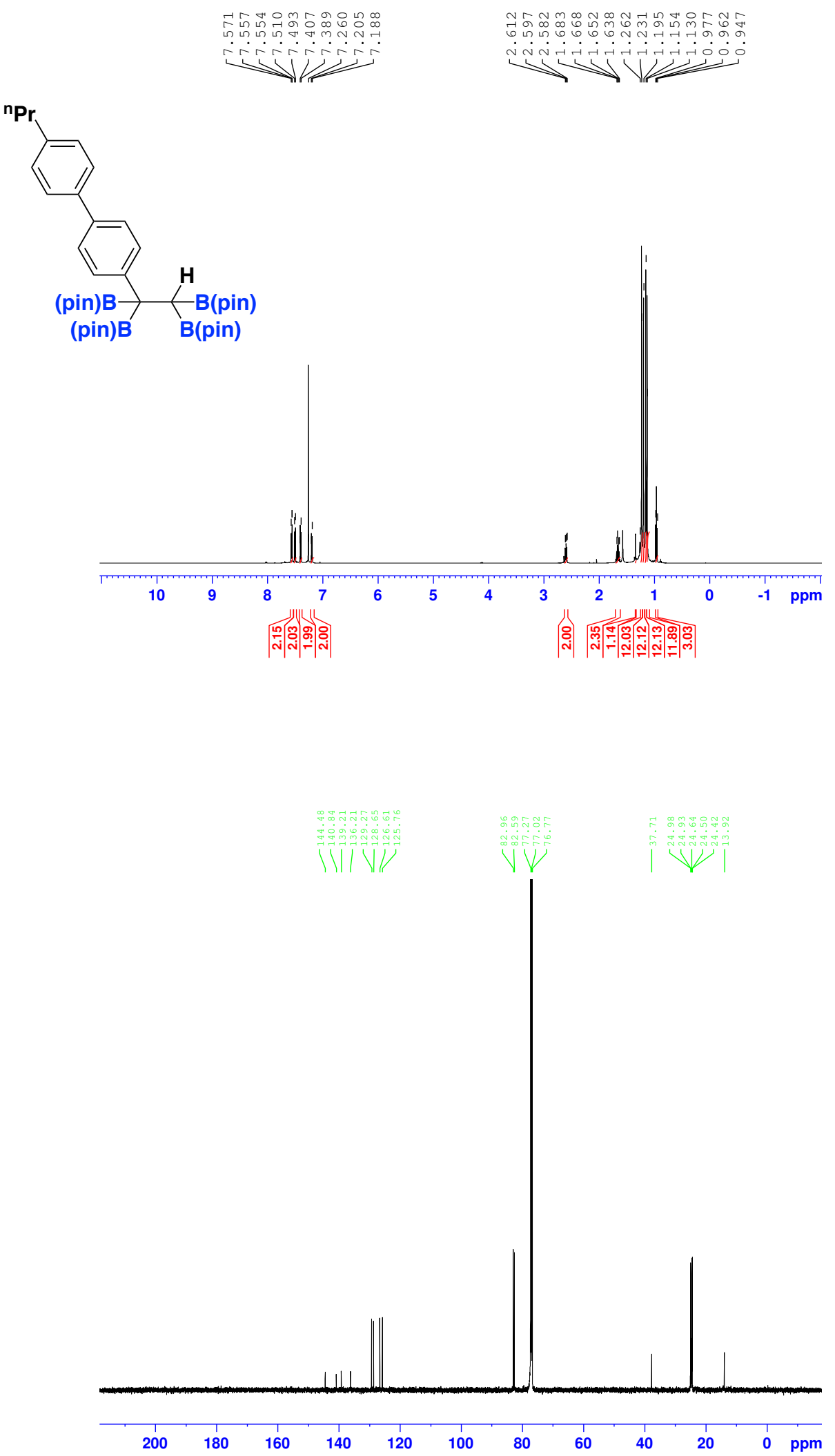

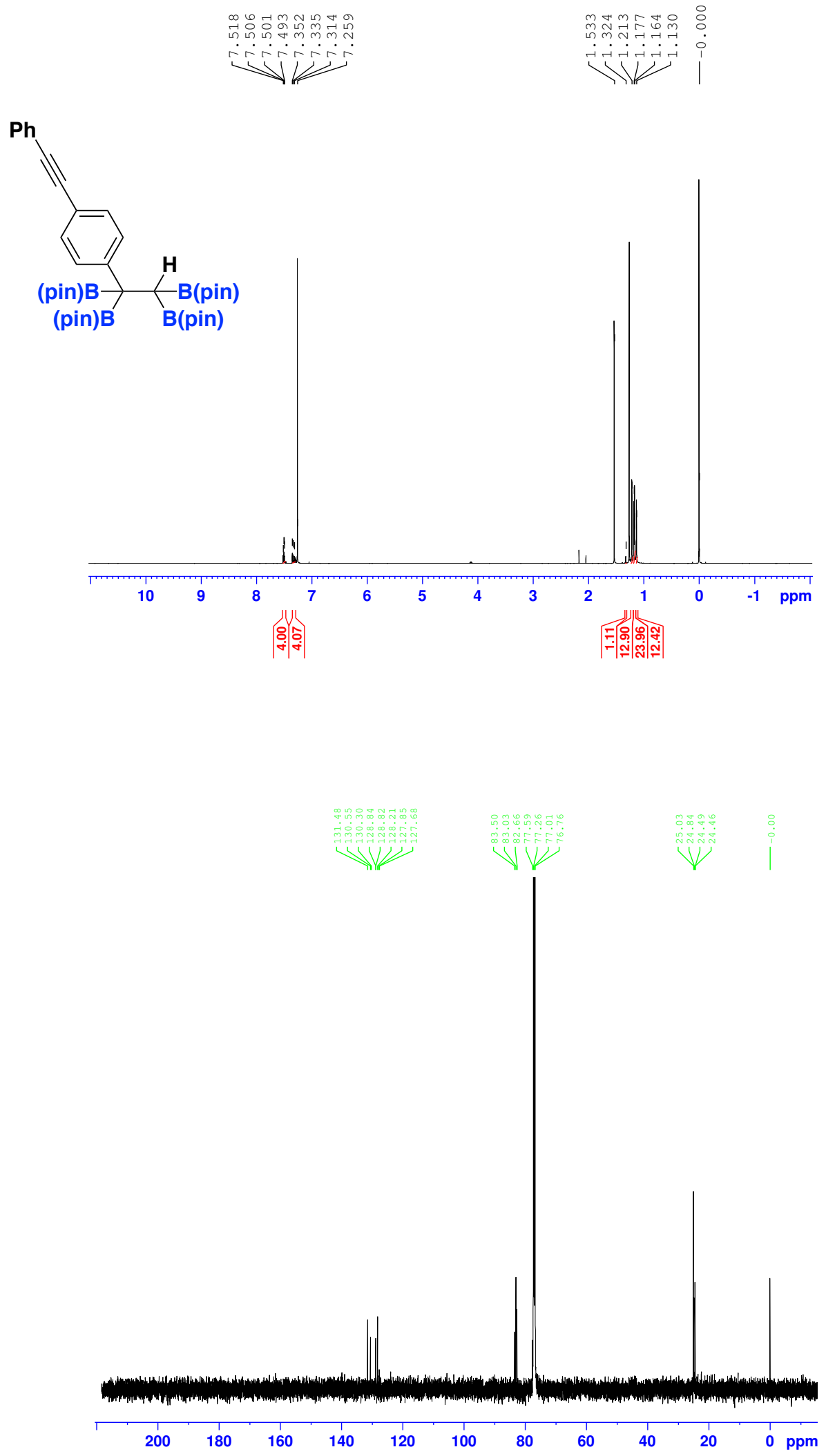

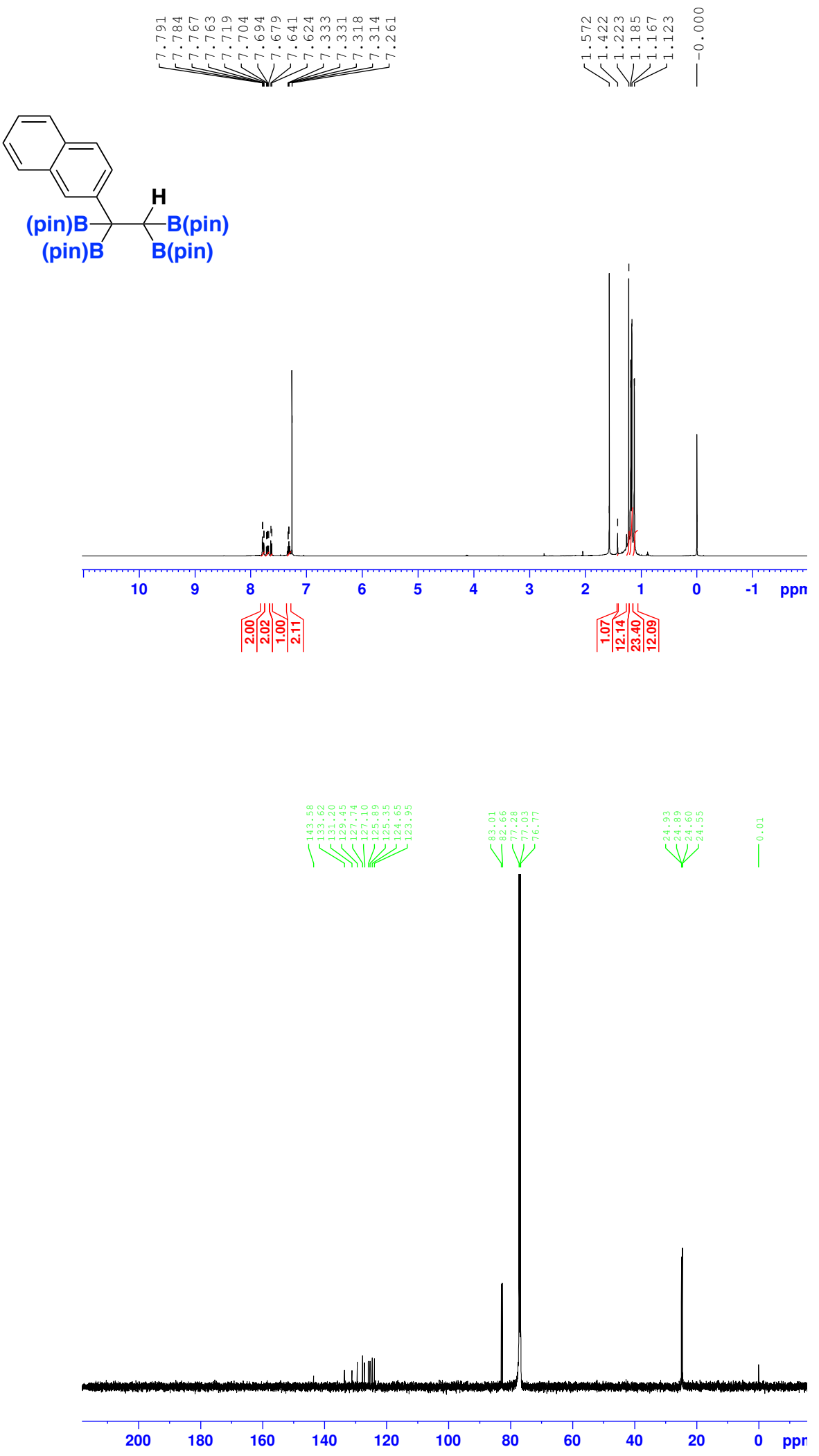

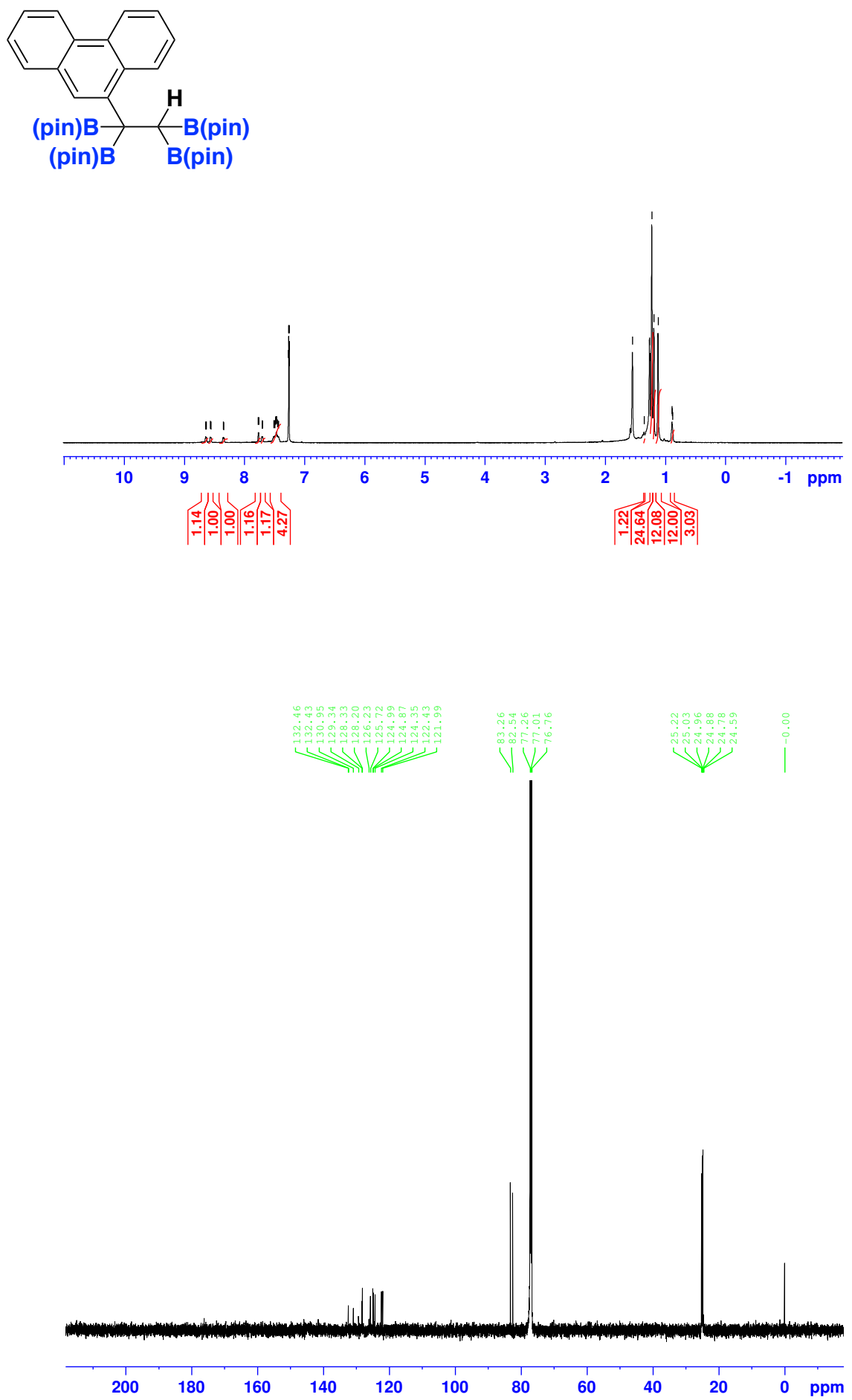


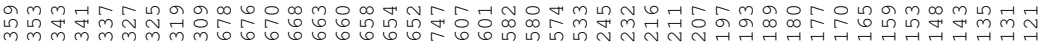

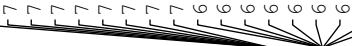
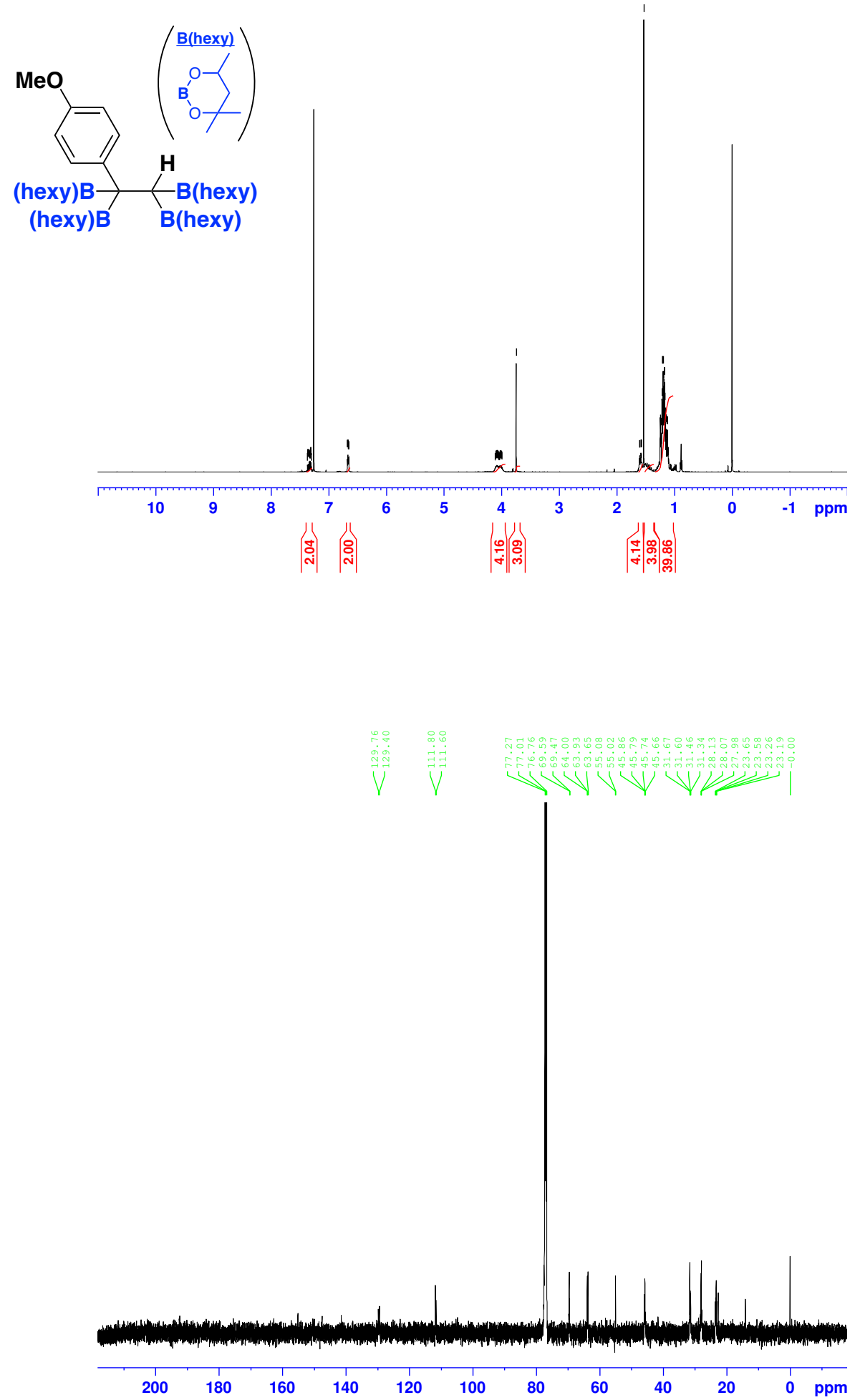

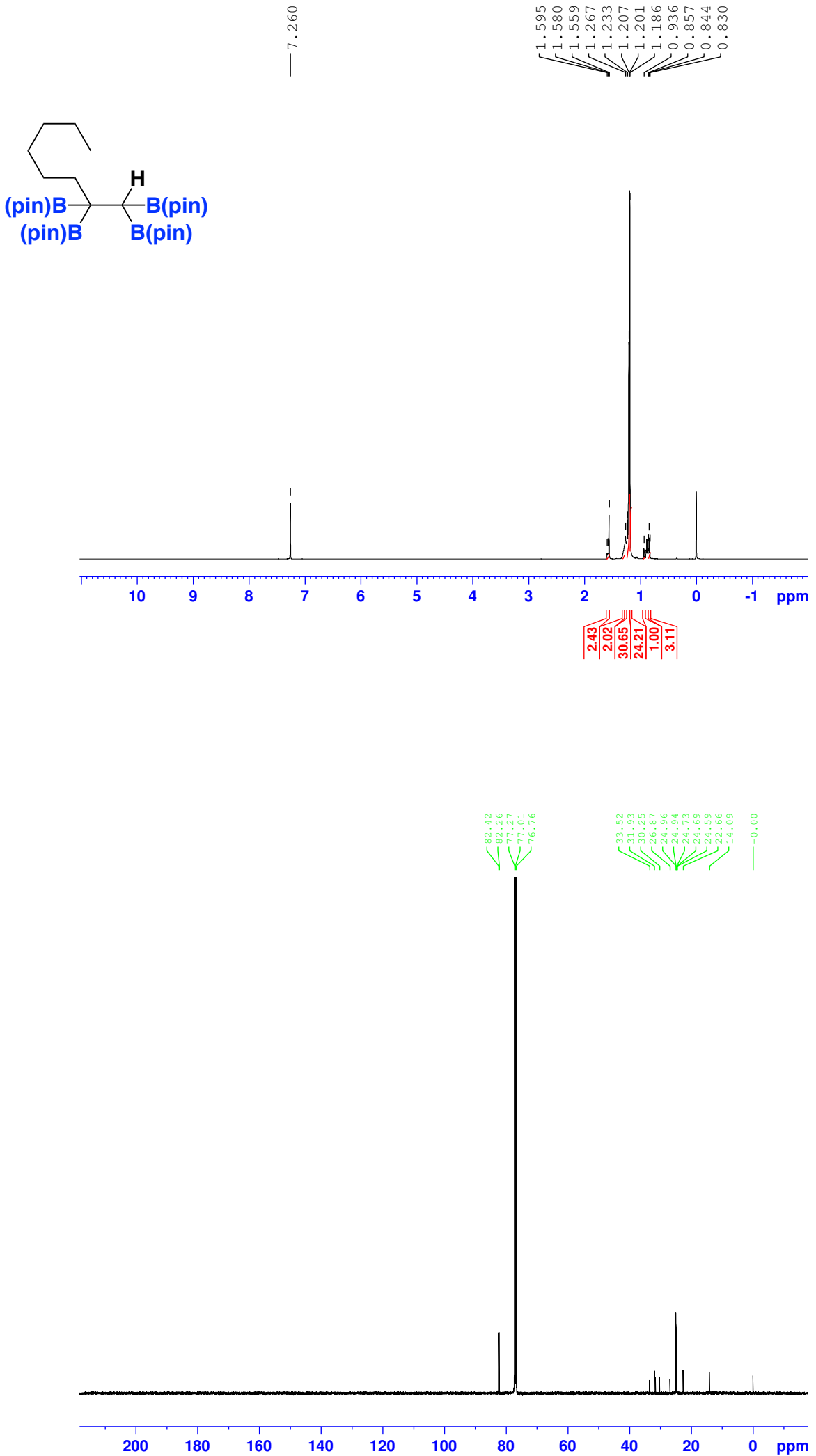

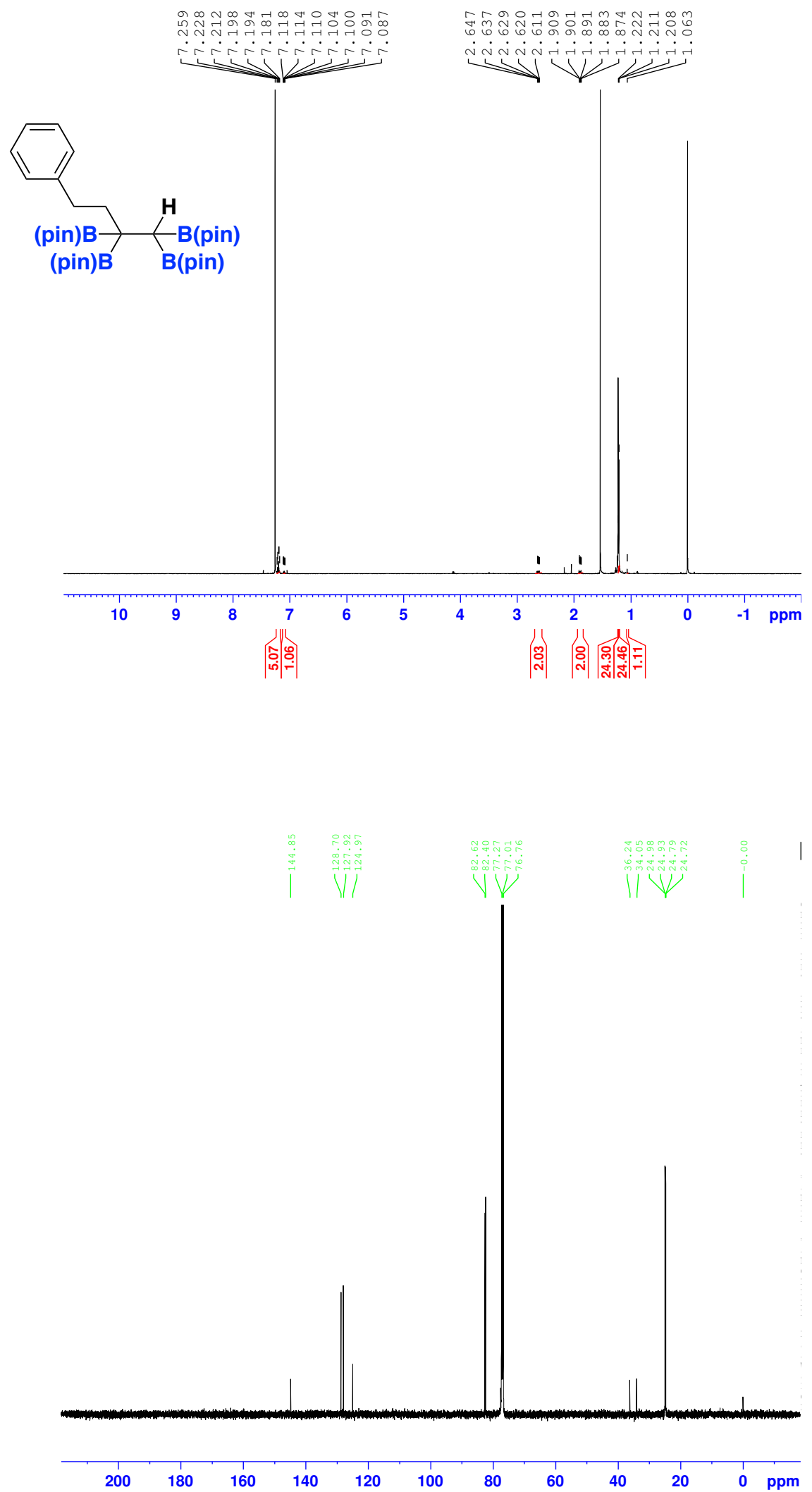

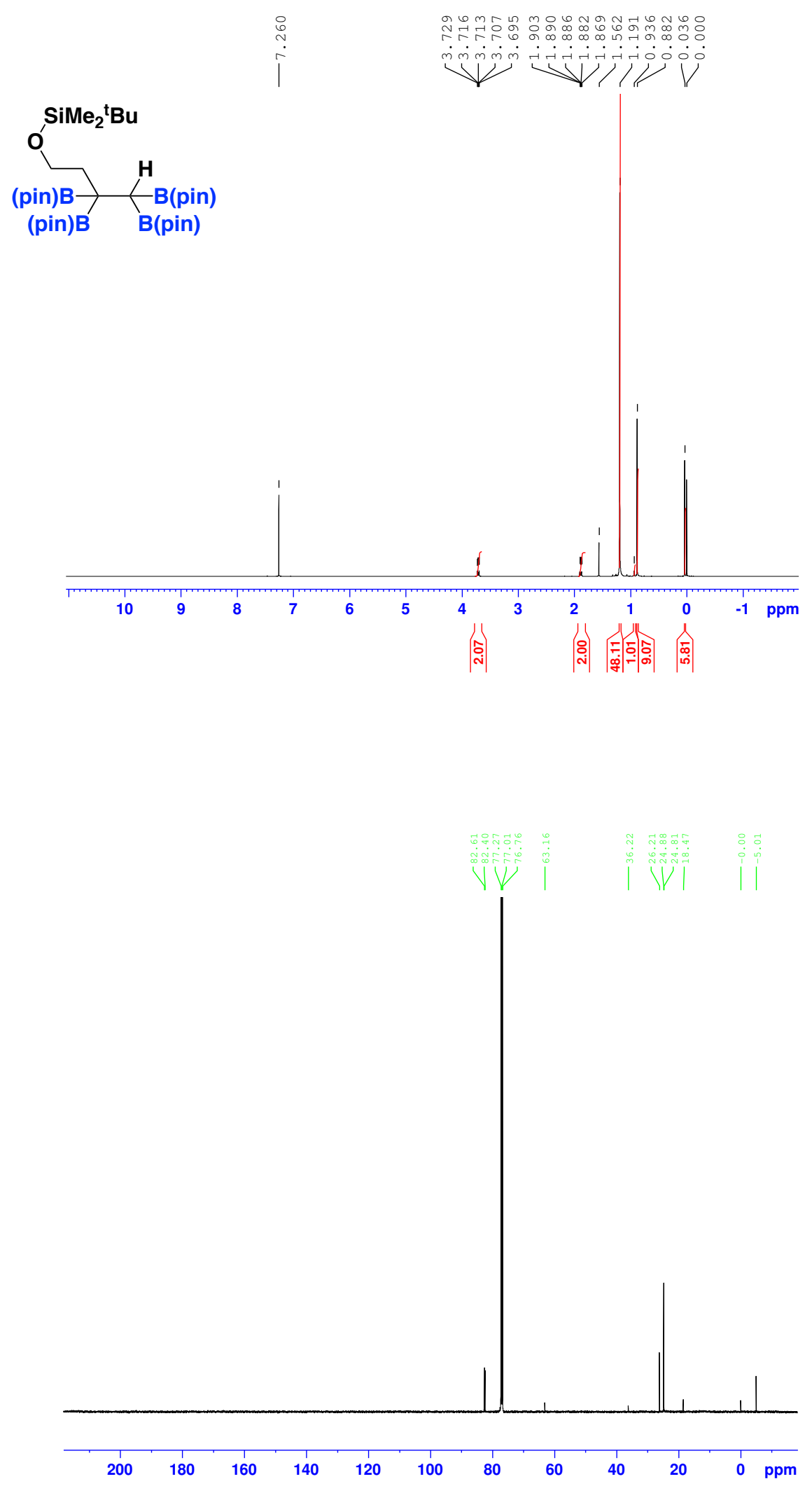

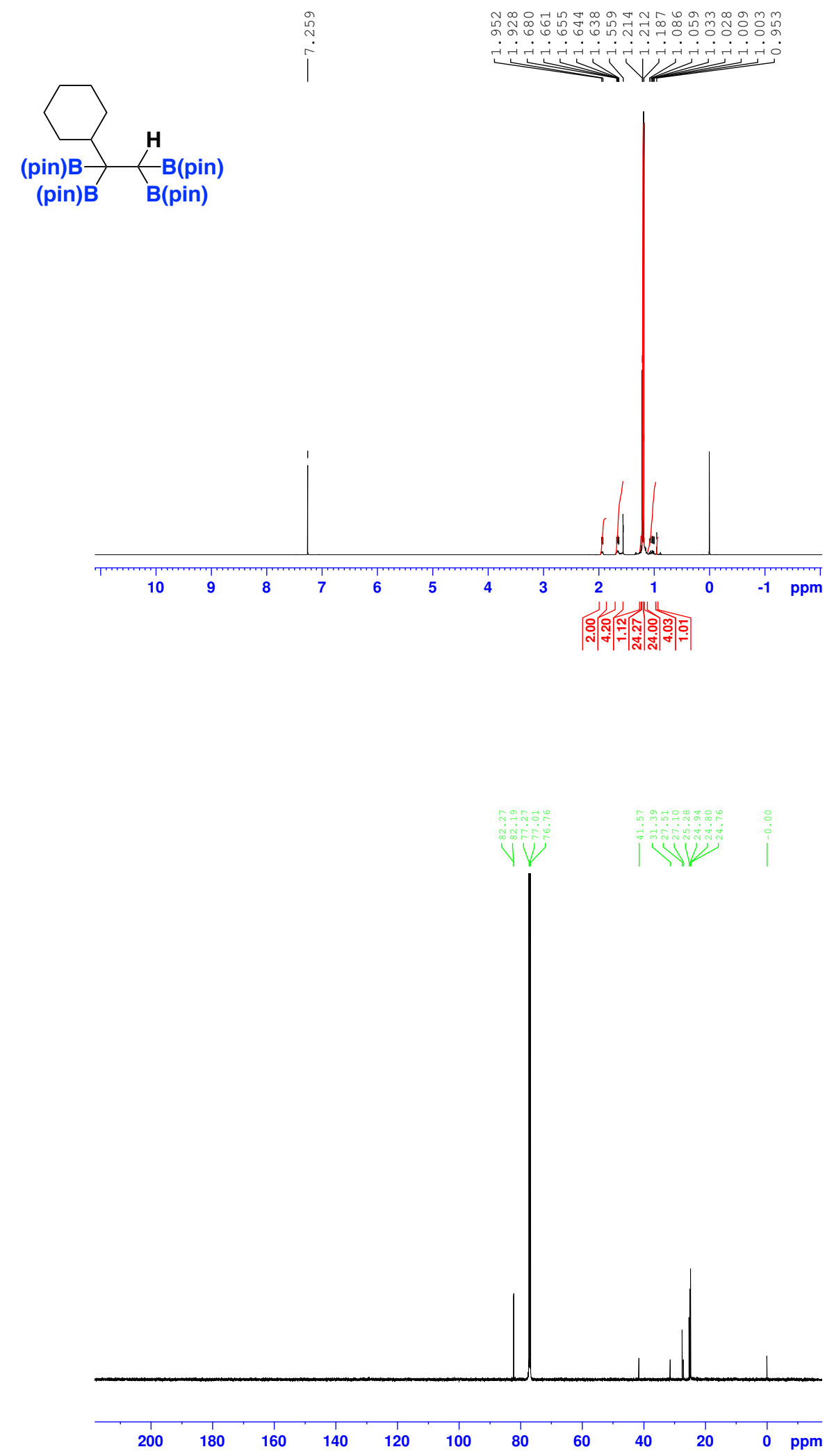

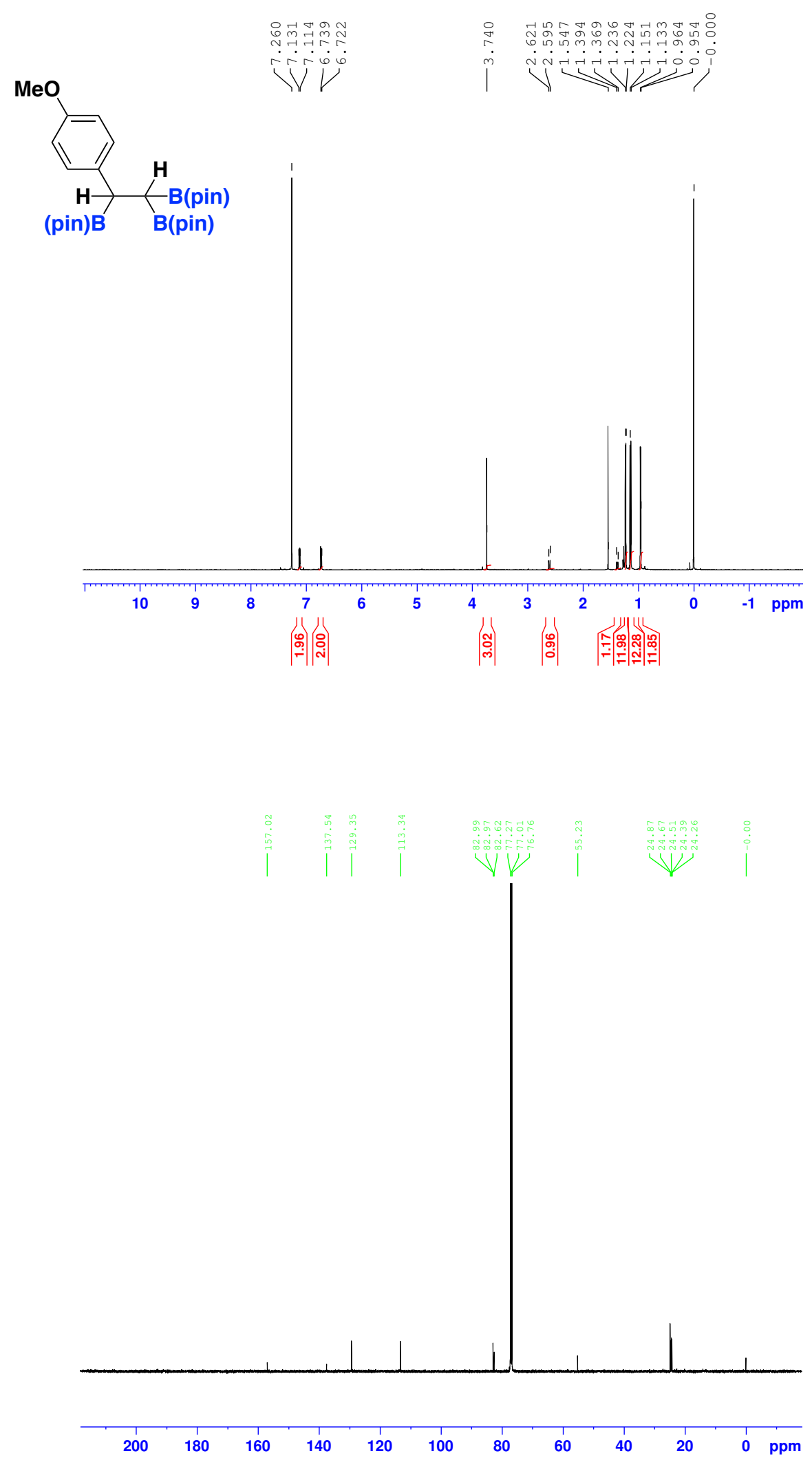

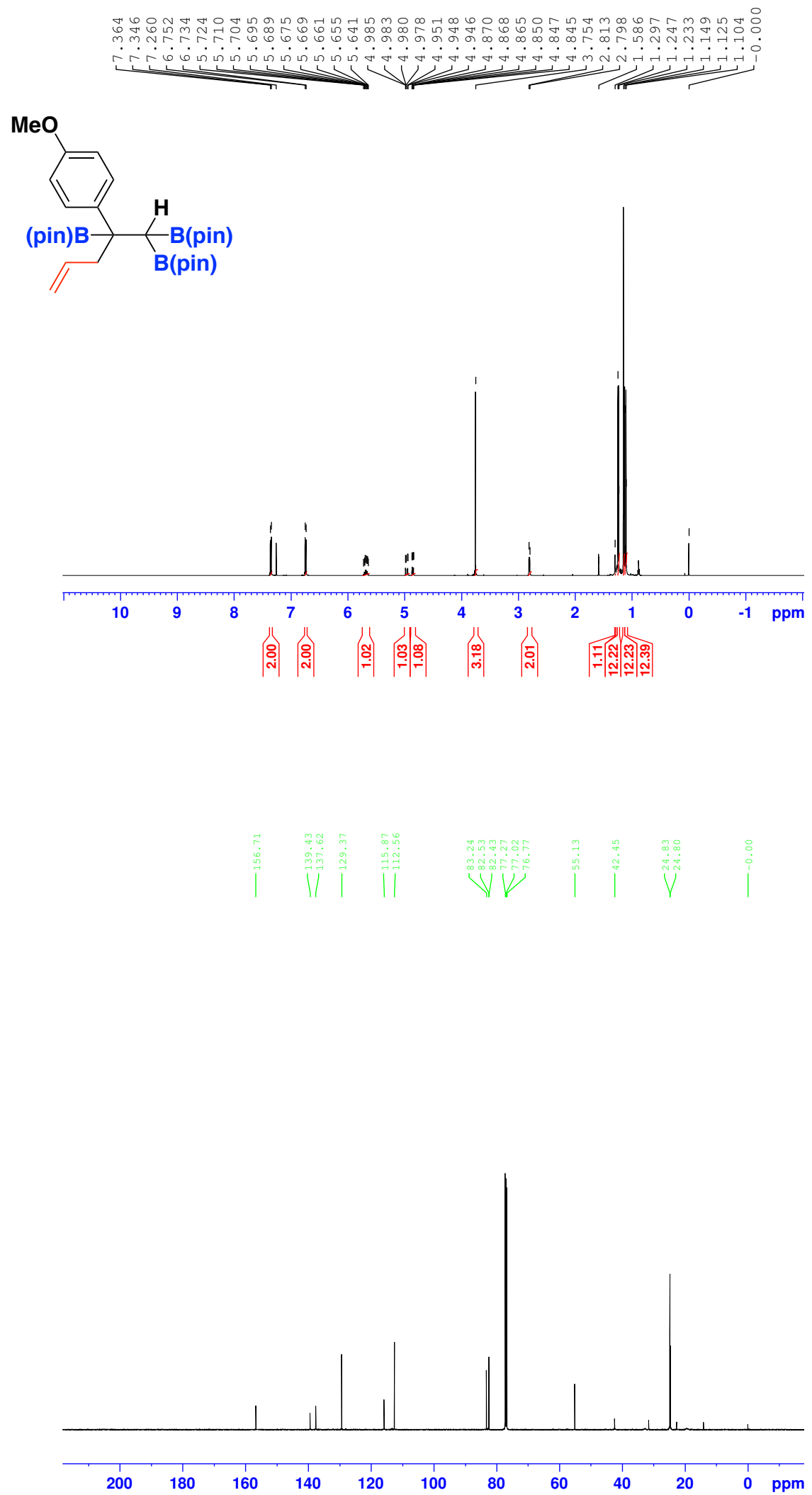

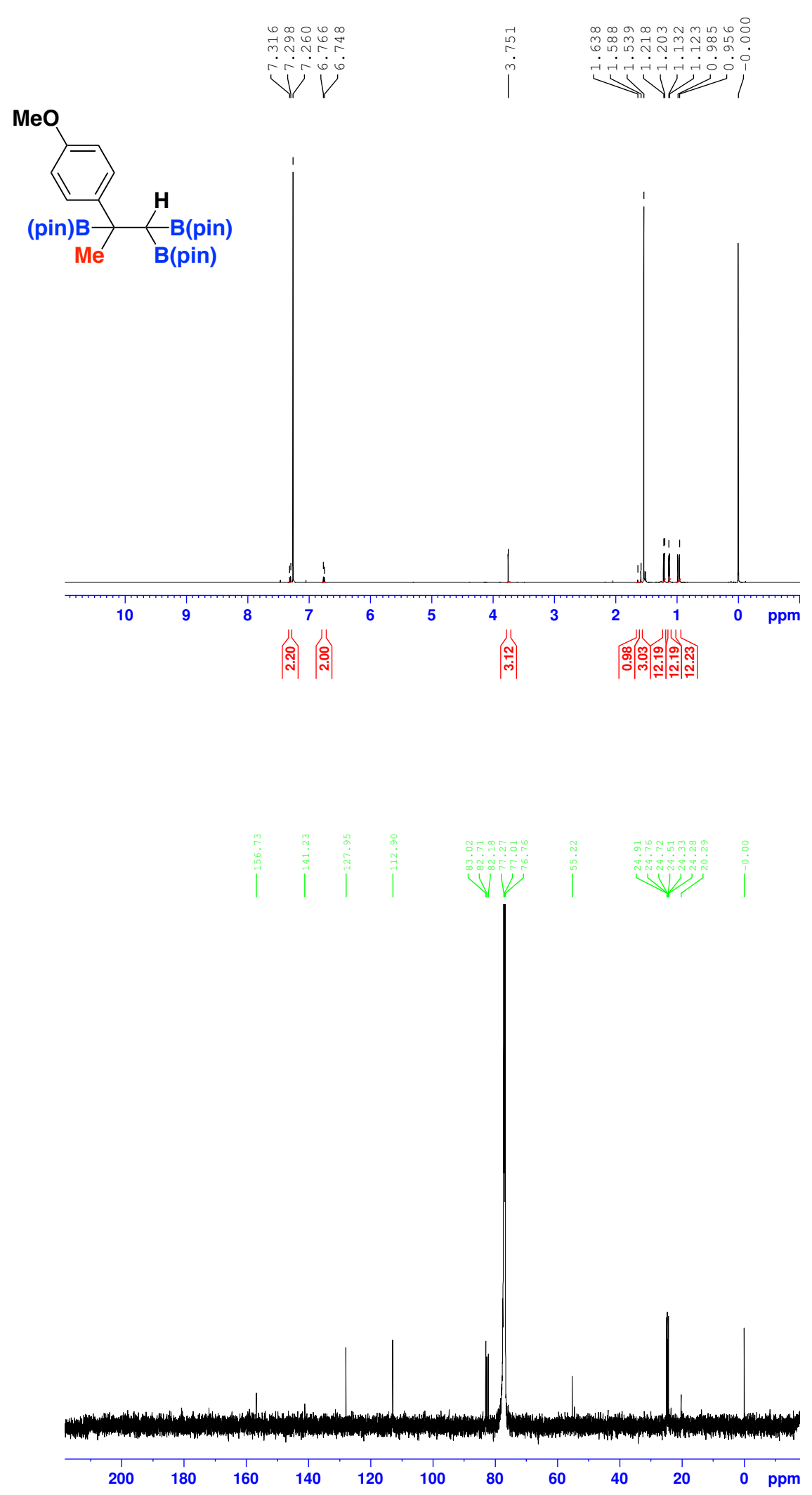

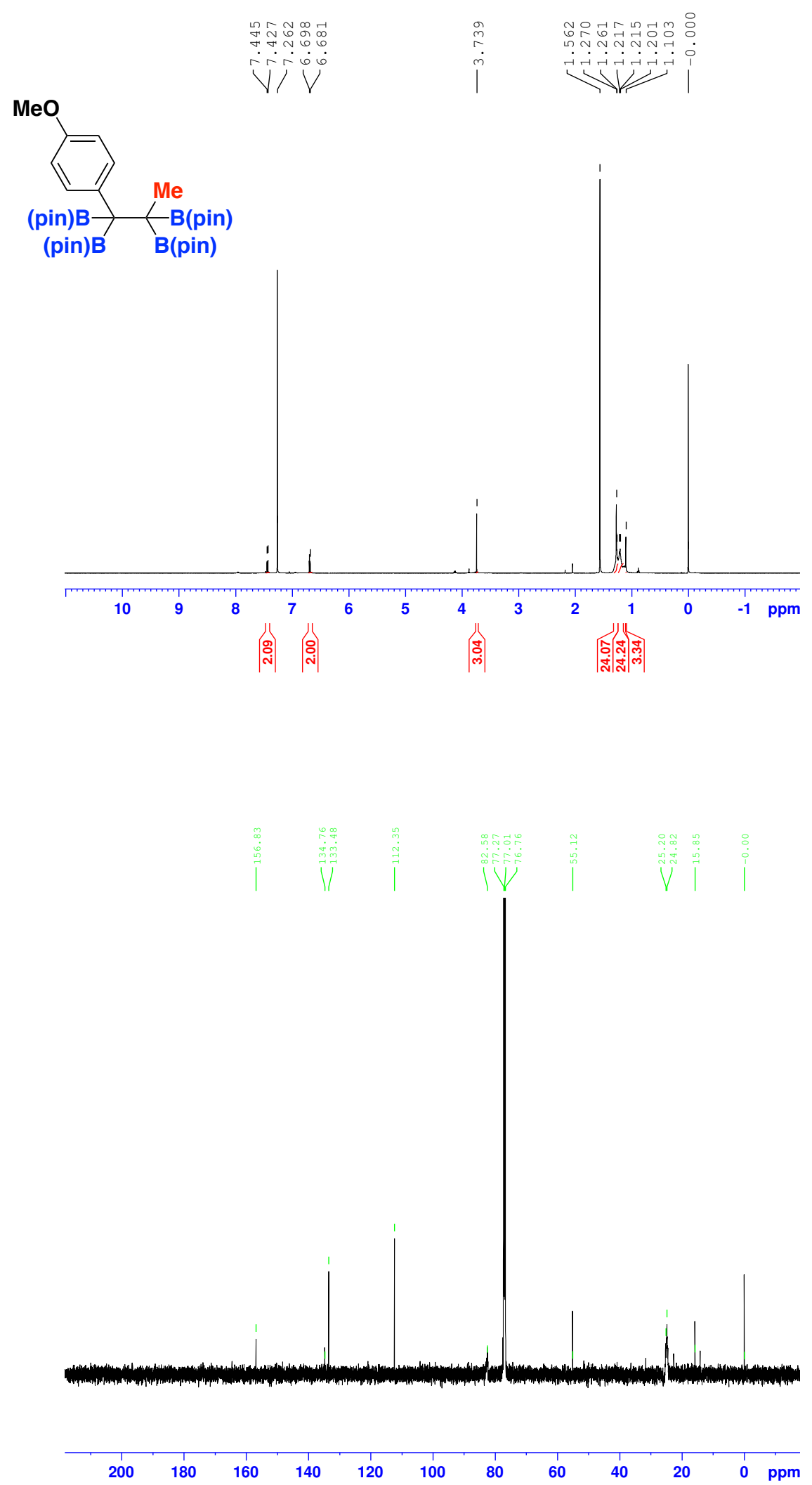

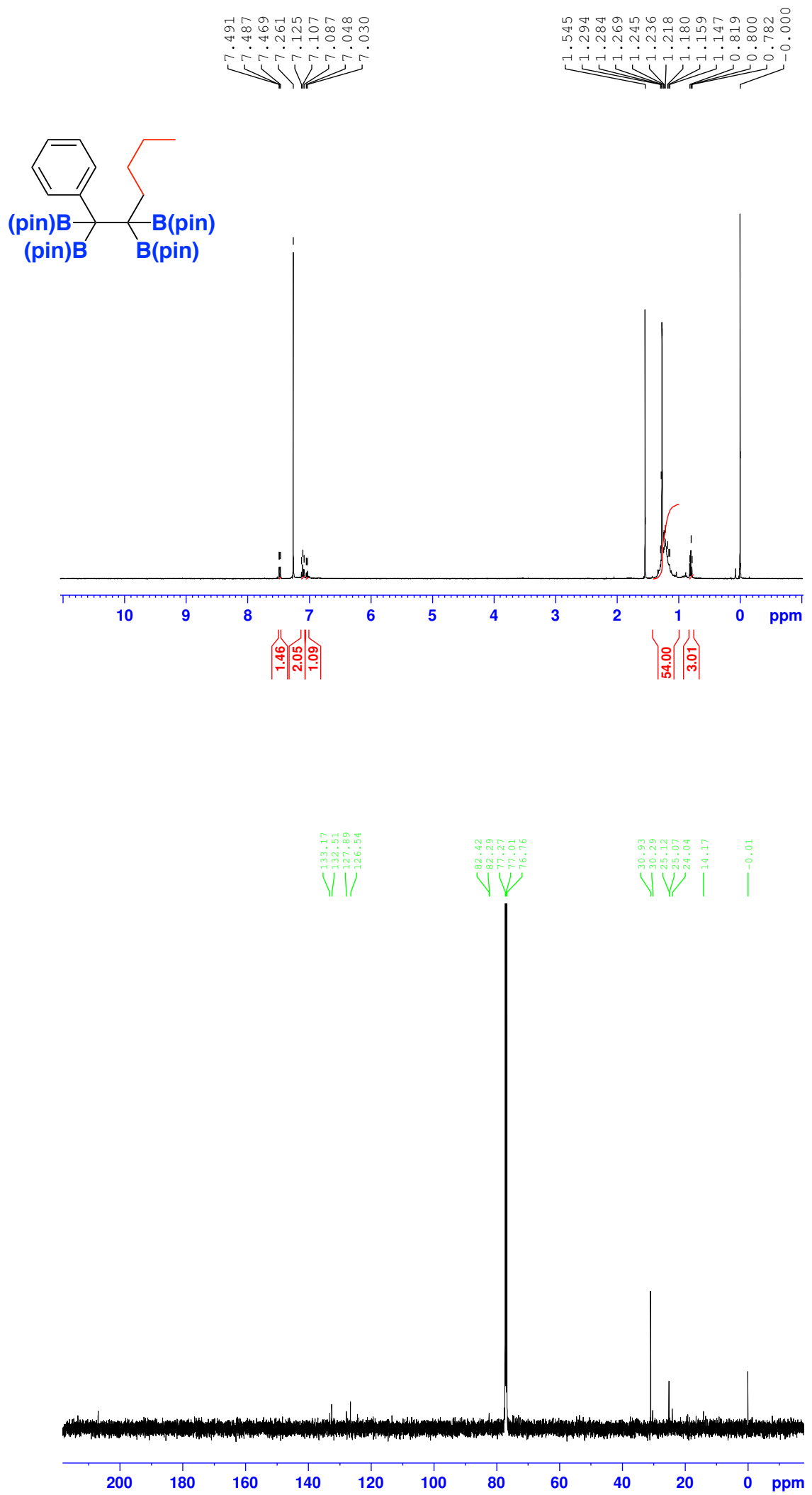

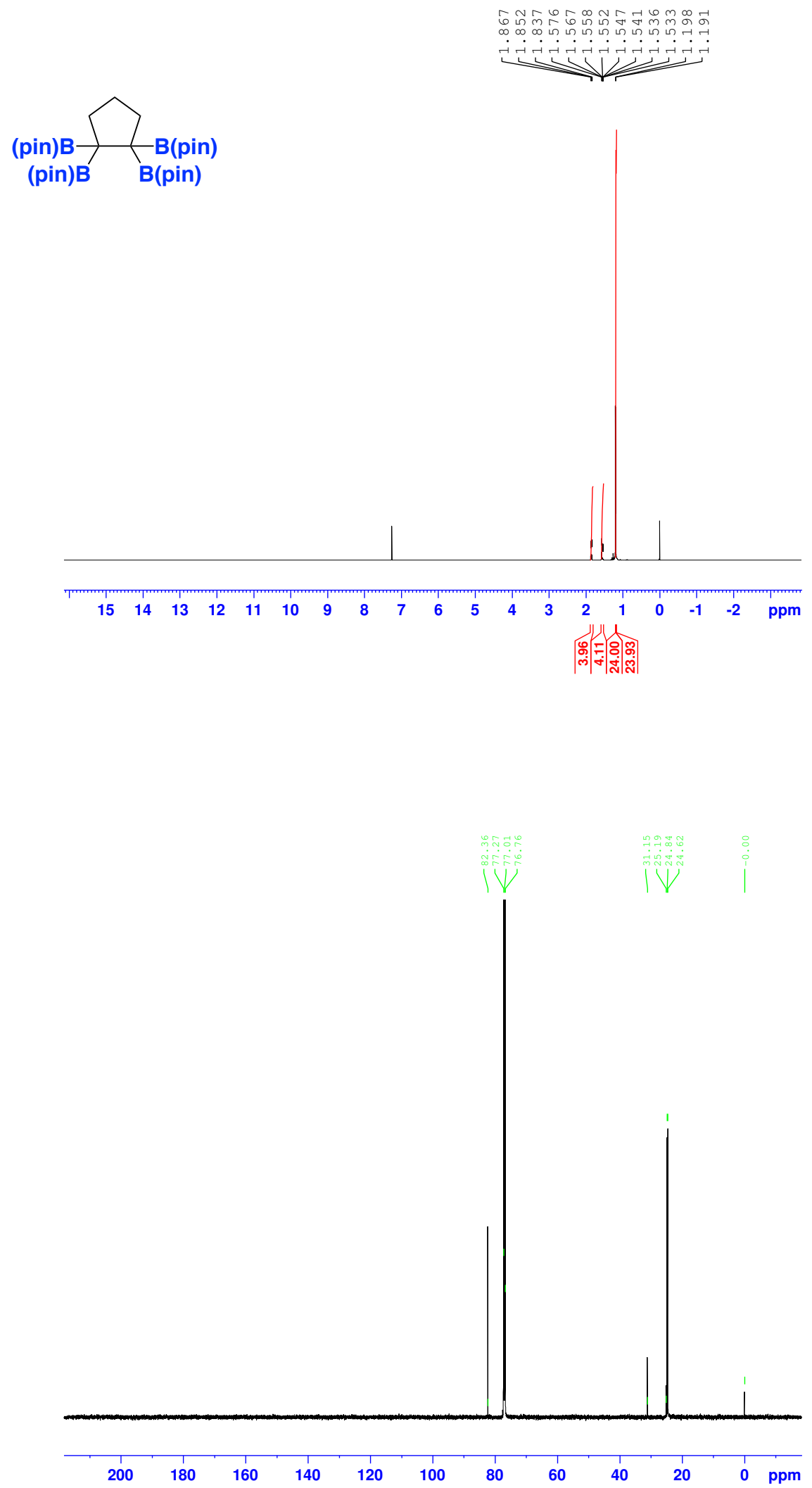